\title{
1 A co-culture microplate for real-time measurement of microbial
}

\section{2 interactions}

4 Charles Jo ${ }^{\mathrm{a}, \mathrm{b}, *}$, David B. Bernstein ${ }^{\mathrm{a}, \mathrm{b},{ }^{* \dagger}}$, Natalie Vaisman ${ }^{\mathrm{c}, \mathrm{d}}$, Horacio M. Frydman ${ }^{\mathrm{c}}$, Daniel

5 Segrèa,b,c,e,f,\#

$7 \quad{ }^{a}$ Department of Biomedical Engineering, Boston University, Boston, MA, USA

$8 \quad{ }^{\mathrm{b}}$ Biological Design Center, Boston University, Boston, MA, USA

$9 \quad{ }^{\mathrm{c}}$ Department of Biology, Boston University, Boston, MA, USA

$10{ }^{\mathrm{d}}$ CAPES Foundation, Ministry of Education of Brazil, Brasília - DF 70040-020, Brazil

11 e Program in Bioinformatics, Boston University, Boston, MA, USA

$12{ }^{\mathrm{f}}$ Department of Physics, Boston University, Boston, MA, USA

13

$14{ }^{*}$ Charles Jo and David Bernstein contributed equally to this work.

$15 \dagger$ Present Address: David Bernstein, Department of Bioengineering, University of California

16 Berkeley, Berkeley, CA, USA

$17{ }^{\#}$ Correspondence: dsegre@,bu.edu 


\section{Abstract}

19 The dynamic structures of microbial communities emerge from the complex network of

20 interactions between their constituent microbial organisms. Quantitative measurements of these

21 microbial interactions are important for understanding and engineering microbial community

22 structure. Here, we present the development and application of the BioMe plate, a redesigned

23 microplate device in which pairs of wells are separated by porous membranes. BioMe facilitates

24 the measurement of dynamic microbial interactions and integrates easily with standard laboratory

25 equipment. We first applied BioMe to recapitulate recently characterized, natural symbiotic

26 interactions between bacteria isolated from the D. melanogaster gut microbiome. Specifically, the

27 BioMe plate allowed us to observe the benefit provided by two Lactobacilli strains to an

28 Acetobacter strain. We next explored the use of BioMe to gain quantitative insight into the

29 engineered obligate syntrophic interaction between a pair of E. coli amino acid auxotrophs. We

30 integrated experimental observations with a mechanistic computational model to quantify key

31 parameters associated with this syntrophic interaction, including metabolite secretion and

32 diffusion rates. This model also allowed us to explain the slow growth observed for auxotrophs

33 growing in adjacent wells, by demonstrating that under the relevant range of parameters, local

34 exchange between auxotrophs is essential for efficient growth. The BioMe plate provides a

35 scalable and flexible approach for the study of dynamic microbial interactions. 


\section{Introduction}

The structures of microbial communities, their composition, diversity, and stability, are emergent properties shaped by the interactions between their constituents (1-5). There are many possible modes of interaction between microorganisms. Direct interactions, which require cell-

41 cell contact, include mechanisms like bacterial conjugation, contact-dependent growth inhibition

42 systems, and intercellular nanotubes (6-9). Microorganisms may also interact via diffusion-

43 mediated mechanisms, such as competition for shared nutrients, production of toxins or

44 communal resources, modulation of environmental conditions, quorum sensing, plasmid

45 exchange, and metabolic cross-feeding (10-14). These multi-faceted interactions within a

46 microbial community have a profound impact on its composition, diversity, and stability $(2,4$,

47 15-17). An appreciation for, and improved understanding of, microbial interactions can elucidate

48 the metabolic and ecological principles of microbial community assembly, as well as facilitate

49 the rational design of novel microbial consortia.

While several approaches have been developed to measure different kinds of inter-

51 microbial interactions, the task of determining the dynamic effect of one microbe's growth on

52 another remains a challenging task (2, 3, 18-20). Mixed co-cultures, where distinct microbes are

53 cultured in the same vessel, are commonplace. However, measuring the abundance of individual

54 members in co-culture is generally difficult and laborious, and requires sampling for CFU

55 counting (5), or imaging, which is usually restricted to fluorescently labelled strains (21). Plating

56 assays are also prevalent, where a microbe is co-cultured on, or in proximity to, a colony of

57 another microbial species (22-24). Conditioned media assays can also be used to determine how

58 the metabolic byproducts and environmental modifications of one microbe affect the growth of

59 another $(4,25)$. However, plating assays are limited by their necessity for phenotypic 
60 differentiation between microbes and inadequate quantitative readout, whereas conditioned

61 media assays are restricted to the study of unidirectional, non-concurrent interactions.

62 Metagenomic and 16S amplicon sequencing have seen increasing usage to measure relative

63 changes in species abundance, but are ultimately restricted by their cost, temporal resolution, and

64 operational challenges $(26,27)$.

65 To address these methodological limitations, systems and devices have been developed to

66 measure co-cultured microbial growth (28). High-throughput microfluidics and micro-droplet

67 based approaches have been designed to screen large numbers of multi-species microbial

68 interactions (29-33). While these approaches offer exciting potential, they can be difficult to

69 apply using standard laboratory equipment, require extensive training and expertise (limiting

70 their accessibility), and often do not allow for the simultaneous observation of the growth of each

71 co-culture member. Alternatively, co-culture systems that utilize a porous membrane to

72 physically isolate individual cultures, and to allow for the exchange of diffusible molecules, have

73 been designed to enable concurrent growth and measurements of distinct microbial cultures (34-

74 37). While these systems restrict microbial interactions to those mediated by diffusible

75 molecules, they have shown promise for culturing previously uncultivated organisms and

76 simultaneously measuring individual growth dynamics in microbial co-cultures. Despite the

77 promise of existing membrane based systems, improvements in their throughput, ease of

78 manufacturing, and ability to seamlessly integrate with common laboratory equipment could

79 enhance their use for the measurement of microbial interactions.

80 In this work, we present the BioMe plate, a 96-well microplate-based co-culture

81 laboratory device developed to facilitate the observation of dynamic microbial interactions.

82 BioMe separates interacting microbial cultures with a porous membrane, physically isolating 
83 individual cultures while allowing for the exchange of diffusible molecules. The growth

84 dynamics of interacting microbial cultures can be measured independently using spectroscopic

85 methods of standard laboratory plate readers. The BioMe plate provides a high-throughput 96-

86 well format and a 3D printed design that facilitates manufacturing and modifications.

We first used BioMe to observe a known symbiotic interaction potentially relevant to the

88 microbiome of a host organism. We chose to study a recently reported symbiosis between

89 organisms of the genera Acetobacter and Lactobacillus isolated from the gut of a Drosophila

90 melanogaster laboratory stock (38). The D. melanogaster gut microbiome is relatively simple,

91 hosting low bacterial diversity (1-30 species). Acetobacter and Lactobacillus are the most

92 commonly found bacterial genera in both lab-reared and wild D. melanogaster flies, affecting

93 development, metabolism, and behavior (39-41). The presence of these two genera has been

94 suggested to contribute to deterministic processes related to the assembly of the D. melanogaster

95 microbiome (42), as they interact through the exchange of metabolic waste products from

96 Lactobacillus to Acetobacter (38). Here we use BioMe to implement pairwise co-cultures

97 between A. oryzifermentans and two distinct Lactobacillus strains (L. plantarum and L. brevis)

98 isolated from lab-reared D. melanogaster stocks (43) providing additional insight into their

99 symbiotic interactions.

100 We next sought to demonstrate the use of the BioMe plate to facilitate the quantitative

101 investigation of a reduced, well-controlled microbial interaction. Specifically, we used the

102 BioMe plate to study the syntrophic interaction between two E. coli amino acid auxotrophs.

103 Engineered auxotrophic bacteria have been used as compelling model systems for the study of

104 microbial interactions due to their well characterized metabolic requirements (44-46). In the

105 work of Mee et al. (2014) E. coli strains were genetically recombineered for a unique single 
106 amino acid auxotrophy and then grown together in mixed co-cultures to identify syntrophic pairs

107 - where distinct amino acid auxotrophs could sustain each other's growth. A syntrophic pair of

108 E. coli auxotrophs from this study, the lysine and isoleucine auxotrophs (referred to as $\Delta$ Lys and

$109 \Delta$ Ile in the text), were co-cultured in the BioMe plate to further characterize and quantify the

110 syntrophic interaction between these two auxotrophs.

111 In parallel to the device itself, we developed a computational model of the growth and

112 nutrient dynamics of two strains grown in connected BioMe wells. We applied this model to the

113 syntrophic E. coli interaction to determine the factors that underlie their co-dependent cross-

114 feeding relationship. In particular, we used this model, alongside an approximate Bayesian

115 computation based approach, to infer plausible ranges for interaction parameters related to the

116 amino acid's diffusion across the membrane and leakage out of $E$. coli cells. Together, these

117 efforts demonstrate the scope of novel questions and experiments that are enabled by the BioMe

118 plate, ultimately improving our understanding of the metabolic interactions and ecological

119 relationships that shape microbial community structure. 


\section{Results}

121 BioMe development

122 We developed a microplate-based co-culture device, the BioMe plate, which enables the

123 quantitative measurement of microbial interactions (Figure 1). A series of vertical porous

124 membranes physically isolate constituent members of pairwise microbial interactions while

125 allowing for the exchange of diffusible molecules. The physical segregation of interacting

126 microbial cultures enables real-time growth dynamics measurements of each microbial

127 population. A range of micro-scale pore sizes can be selected from, depending on the user's

128 desired application, molecule size selectivity, and degree of diffusion across the membrane.

129 The BioMe plate is comprised of several components, which are further detailed in

130 Supplemental Table 1. The standard 96-well microplate design is fragmented into six discrete

131 body segments, which form the wells of the device. Each well has an opening on the side of the

132 body segment that connects it to its respective co-culture well in a separate body segment, via O-

133 ring and porous polycarbonate membrane. The body segments are laterally fastened and sealed

134 using rods and nuts, yielding a total capacity of 30 pairwise co-culture assays. Of note, the body

135 segments can be machined from polypropylene or stereolithography (SLA) 3D printed using a

136 biocompatible and autoclavable dental resin, circumventing the need for labor-intensive

137 machining. A transparent base forms the bottom of the plate, allowing for real-time

138 spectroscopic readings (optical density or fluorescence) of each well. The base is machined from

139 polycarbonate sheet and is vertically fastened and sealed to the assembled body chassis via a

140 laser-cut gasket and screws. This forms the core of the BioMe plate, which is housed in between

141 two clear 96-well plate lids to prevent evaporation and equipment damage if leakage were to

142 ever occur. 
bioRxiv preprint doi: https://doi org/10.1101/2021 01.07.425753; this version posted January 8,2021 . The copyright holder for this preprint (which was not certified by peer review) is the author/funder, who has granted bioRxiv a license to display the preprint in perpetuity. It is made available under aCC-BY-NC-ND 4.0 International license.

A

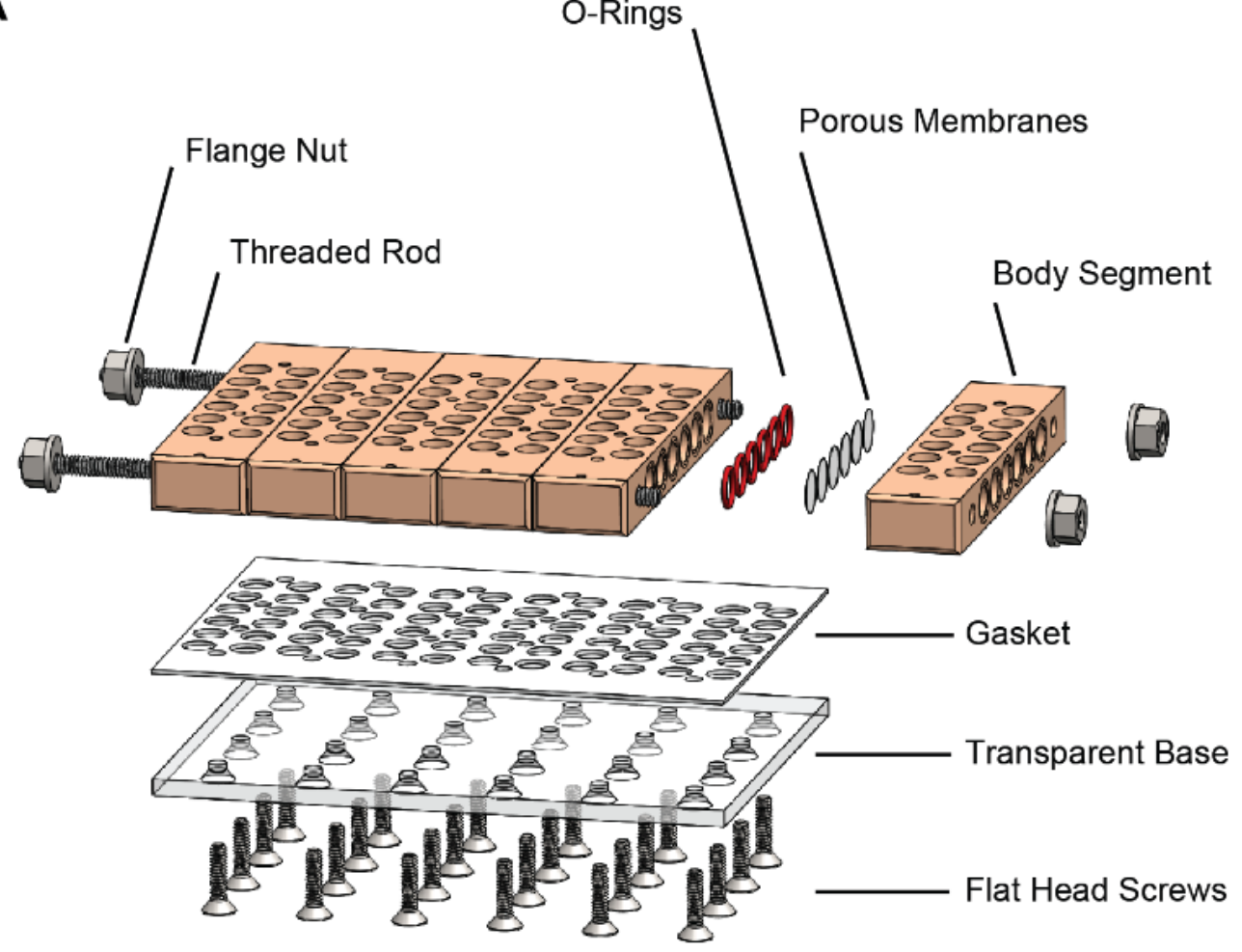

$\mathrm{B}$
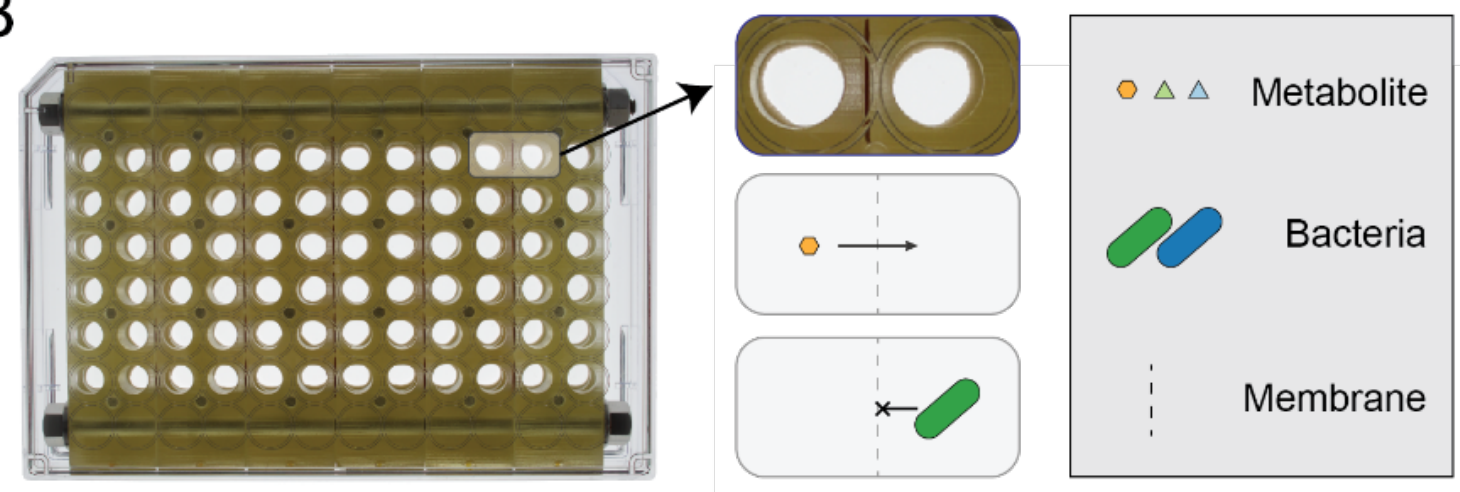

C
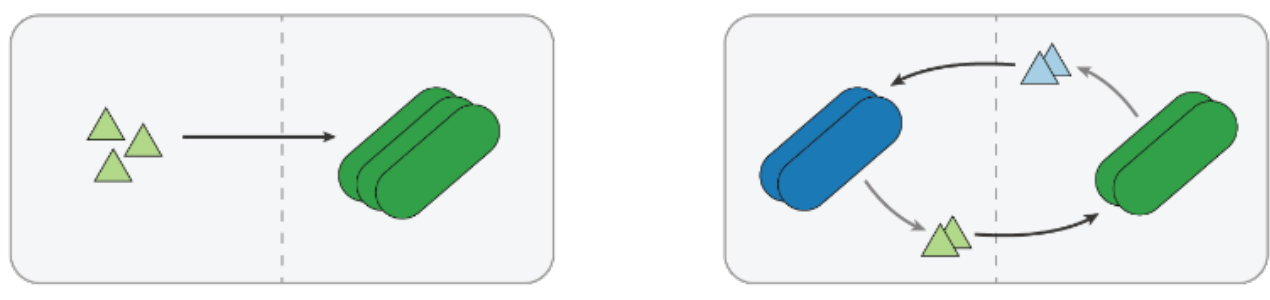


\section{Figure 1: The BioMe Plate}

145 (A) Exploded view of the computer-aided design of the BioMe plate. The different components

146 are assembled to form a microplate-based co-culture device. (B) Photograph of the fully

147 assembled BioMe plate (top view). Adjacent wells are separated by a porous membrane that

148 allows the diffusion of metabolites and small molecules but not microbes. (C) The BioMe plate

149 enables a variety of microbiological experiments. This includes metabolite growth assays (left) to

150 observe microbial population growth when seeded across from a metabolite, and co-culture

151 assays (right) to characterize and quantify the effects of co-cultured growth between a pair of

152 interacting microbial cultures. 
A sterilized BioMe plate can be reused to ensure cost-effective, contamination-free repeated use. The porous membranes are disposable and replaced per use. Leakage tests

156 demonstrated a water-tight seal throughout (see Methods, section: Leakage Test) and sterilization

157 validation tests demonstrated successful de-contamination of the BioMe plate (see Methods, 158 section: Sterilization Protocol and Validation). The ability of various membrane pore-sizes to 159 restrict crossover of microbial cells was tested; crossover was observed in several experiments, 160 especially for larger pore sizes $(0.4 \mu \mathrm{m})$ and rarely for smaller pore sizes $(0.03,0.1,0.2 \mu \mathrm{m})(\mathrm{see}$ 161 Results, section: Syntrophic E. coli Amino Acid Auxotroph Interaction, and Discussion). Small molecule diffusion across the porous membranes was tested using colorimetric

163 assay dyes. Specifically, the BioMe plate was loaded with two different dyes, phenol red and 164 bromocresol purple, and their diffusion across membranes with varying pore sizes was 165 monitored (Figure 2A and S2.1). Phenol red and bromocresol purple are typically used as $\mathrm{pH}$ 166 indicators, but when measured at their isosbestic points, 478nm and 490nm, respectively (47, 167 48), their concentrations can be inferred from optical density (absorbance measurements). We

168 developed calibration curves for each dye to ensure accurate extrapolation of concentration from 169 optical density (Supplemental Figure S2.2). Both dyes were observed to diffuse across the 170 porous membrane with a diffusion rate that increased monotonically for increasing pore size, and 171 were not observed to diffuse across a membrane with no pores. The raw optical density data for 172 the diffusion and calibration experiments, laid out as they were on the BioMe plate, are shown in 173 supplemental Figures S2.3 and S2.4.

174 Diffusion coefficients of all tested membrane pore sizes for each chemical dye were 175 inferred using a gradient-driven diffusion model (further described in the Computational 
176 Modeling section). This model gives rise to an exponential function describing the time-

177 dependent concentration of the molecules in either well of the system, as the dyes diffuse across

178 the membrane (Supplemental Equations S1). An exponential function was fit to the dye diffusion

179 data (See Methods and Figure S2.5) to infer a range of estimates for our model's diffusion

180 constant for both dyes and various pore sizes: $0.03,0.1,0.2$, and $0.4 \mu \mathrm{m}$. As shown in Figure $2 \mathrm{~B}$,

181 the mean diffusion constant increases monotonically and non-linearly with increasing pore size.

182 Interestingly, the estimated diffusion constant was consistently higher when calculated using the

183 curves from the well where the dye was initially placed as opposed to when using the curves

184 from the opposite well into which the dye diffused and this effect was more pronounced for the

185 larger pore sizes and for phenol red (see Discussion for additional details). 

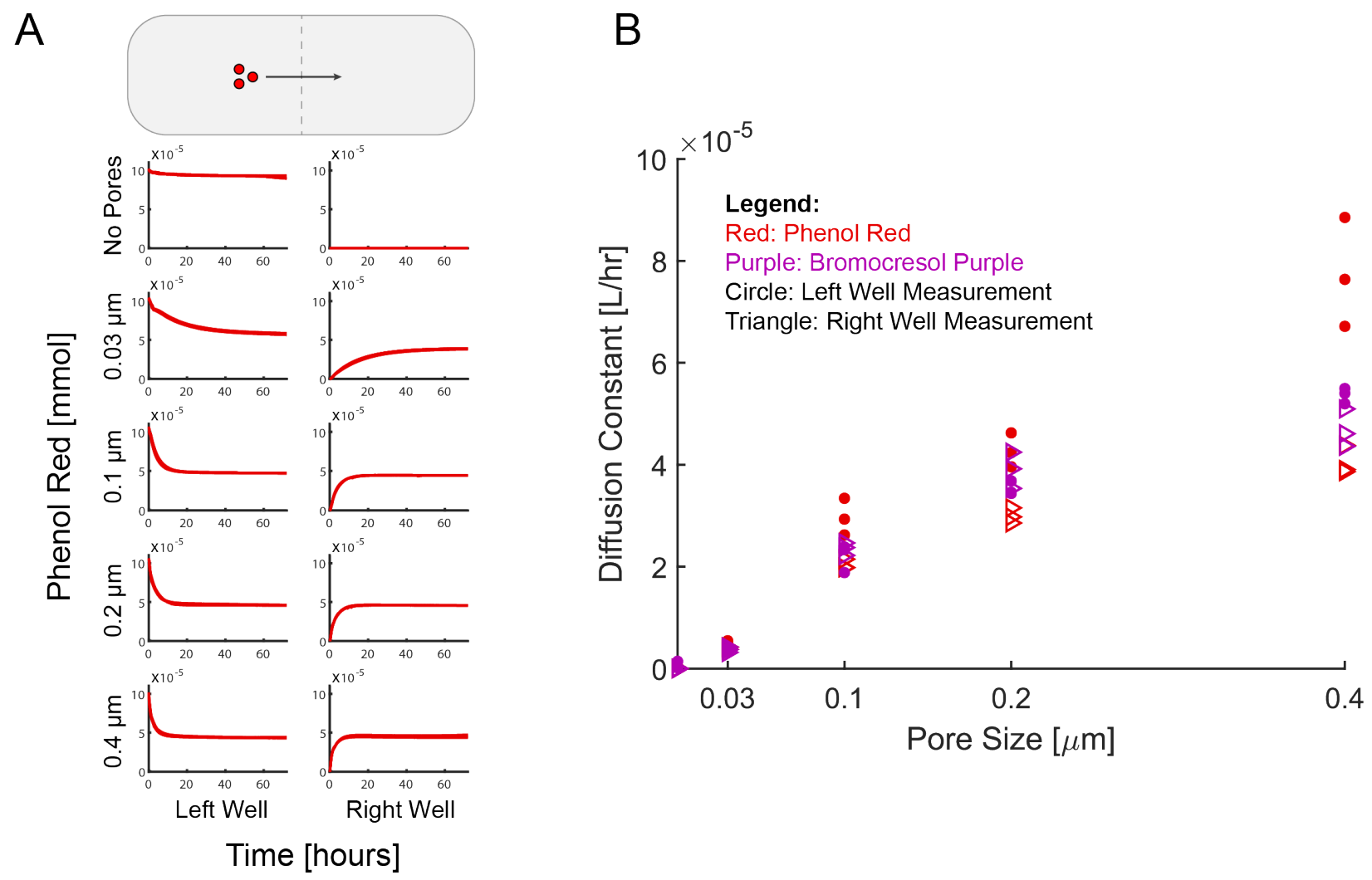

Figure 2: Chemical Dye Diffusion Across Membranes with Variable Pore Size in the BioMe

Plate

189 A small-molecule dye $(400 \mu \mathrm{M}$ concentration of phenol red or bromocresol purple) was loaded

190 into columns of wells to the left of the membrane in the BioMe plate, for varying membrane pore

191 sizes: no pores, $0.03,0.1,0.2$, and $0.4 \mu \mathrm{m}$. Optical density at isosbestic point (478nm for phenol

$192 \mathrm{red}, 490 \mathrm{~nm}$ for bromocresol purple) was measured for $72 \mathrm{hrs}$, in $15 \mathrm{~min}$ intervals. Dye

193 concentration was inferred by linear calibration to optical density. (A) Time course of calibrated

194 dye concentration for phenol red with membranes of different pore sizes. Replicates of $\mathrm{n}=3$ (all

195 overlapping curves are shown on the plot) were conducted for each pore size. (B) Diffusion

196 constants for phenol red and bromocresol purple for varying membrane pore sizes, calculated by

197 fitting to dye diffusion model using data from both the left and right well measurements. 
Symbiotic interaction between Drosophila melanogaster gut microbiome species

As a first biological application of the BioMe plate, we confirmed a recently

201 gut microbiome. In particular, we focused on three distinct bacterial strains previously isolated

202 from laboratory-bred flies: one species of Acetobacter (A. oryzifermentans) and two species of

203 Lactobacillus (L. plantarum and L. brevis) (43). The BioMe plate was used to test and observe

204 every pairwise co-culture combination between these three strains. Additionally, relevant

205 controls were included in the experiments, i.e. organisms paired with themselves (self-control)

206 and organisms paired with wells containing growth medium but no other organism (media-

207 control) (Figure 3, Supplemental Figure S3.1 and S3.2). A 0.03 $\mu$ m pore size membrane was

208 chosen to allow exchange of metabolites but ensure no microbial cross-over, as done for the

209 iChip device (49).

210 The co-culture experiments revealed an increased growth of $A$. oryzifermentans when co-

211 cultured with either Lactobacillus strain, demonstrating a clear benefit relative to its growth

212 when co-cultured with itself or no microbe in the opposing well. Interestingly, for both L. brevis

213 and L. plantarum, their growth in co-culture with the Acetobacter strain was similar to their

214 growth in respective media-controls, but still demonstrated improved growth relative to both

215 their self-controls and co-culture with the other Lactobacillus strain. Our results, uniquely

216 enabled by the BioMe plate, suggest that the Acetobacter population disproportionately benefits

217 from this metabolite-mediated interaction. BioMe could serve as the starting point for further 218 exploration of this cross-species interaction, complementing existing approaches $(38,50,51)$. 
A
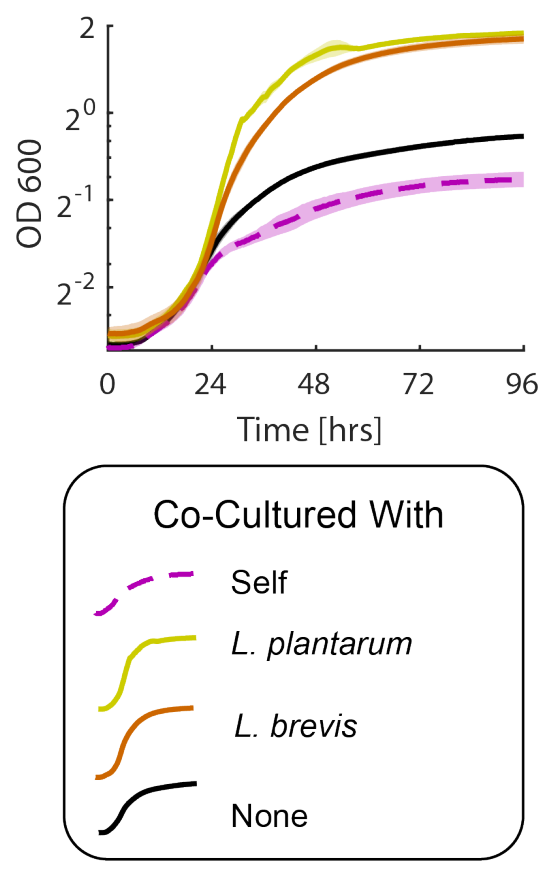

B
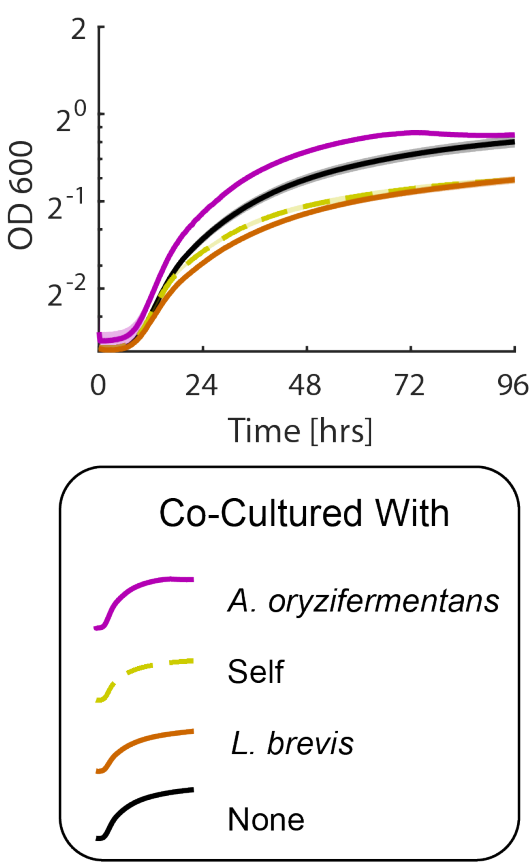
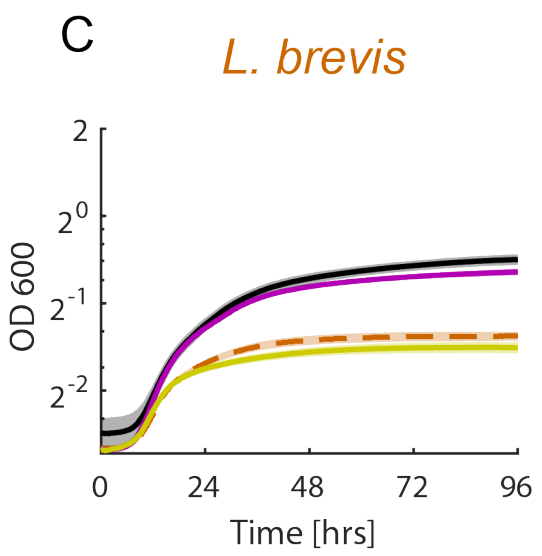

Co-Cultured With

A. oryzifermentans

L. plantarum

Self

None

Figure 3: Commensal Relationship between Drosophila melanogaster Gut-Associated

\section{Microbes}

223 Pairwise co-cultures between all combinations of Acetobacter oryzifermentans (A),

224 Lactobacillus plantarum (B), and Lactobacillus brevis (C). Growth curves are plotted on a log

225 base 2 scale. Membrane pore size of $0.03 \mu \mathrm{m}$ was used throughout these experiments. Lines

226 indicate mean growth curves and shaded regions indicate standard error $(\mathrm{n}=4$ for cross-species

227 co-cultures, $\mathrm{n}=3$ for media-control "none", $\mathrm{n}=6$ for self-control "self" for L. plantarum and $L$.

228 brevis and $\mathrm{n}=4$ for "self" for $A$. oryzifermentans cultures as two outliers were left out, see

229 supplemental figures S3.1 and S3.2 for additional details). 
Next, we used BioMe to enable a more in-depth and quantitative study of a model

232 microbial interaction in which two strains have been engineered to exchange essential

233 metabolites in order to grow. Specifically, we studied the syntrophic interaction between a pair

234 of E. coli amino acid auxotrophs: $\Delta$ Lys and $\Delta$ Ile. These strains were engineered to be unable to

235 biosynthesize lysine or isoleucine, respectively, requiring supplementation of the missing amino

236 acid to grow in monoculture. Despite their inability to grow in monoculture without

237 supplementation, both strains are able to grow when placed together in a mixed co-culture (44).

238 The first test of this system in the BioMe device was a validation that amino acids could

239 diffuse across membranes of various pore sizes and support the growth of an auxotrophic E. coli

240 strain in the adjacent well. Figure 4 shows an example of this validation for lysine diffusion with

241 the $0.1 \mu \mathrm{m}$ pore size. Lysine was shown to diffuse across the membrane, with comparable growth

242 to the positive control where lysine was added into the same well as the microbial culture (Figure

$2434 \mathrm{~A}$ and B). A negative control experiment, lacking the supplemental amino acid, displayed no

244 growth, and thus confirmed the amino acid auxotrophy (Figure 4C). A second negative control

245 experiment, displaying lack of growth for membranes with no pores, confirmed the integrity of

246 the device seal (Figure 4D). These results were repeated with $\Delta$ Ile and isoleucine, and for

247 varying pore sizes: no pores, $0.03,0.1,0.2$, and $0.4 \mu \mathrm{m}$. We found similar results in all cases,

248 with comparable growth between diffusion and positive control conditions and no growth in

249 either of the negative controls (Figure S4.1, S4.2, and S4.3). 

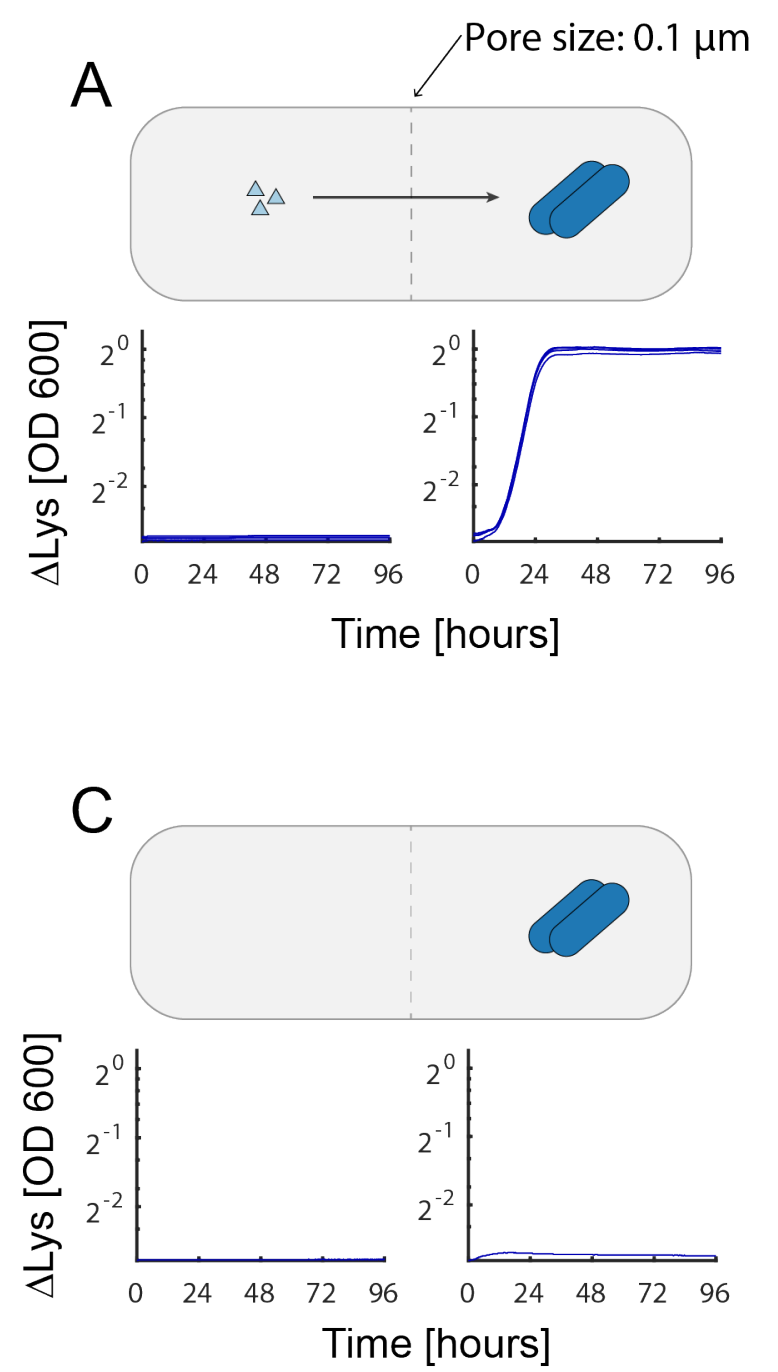
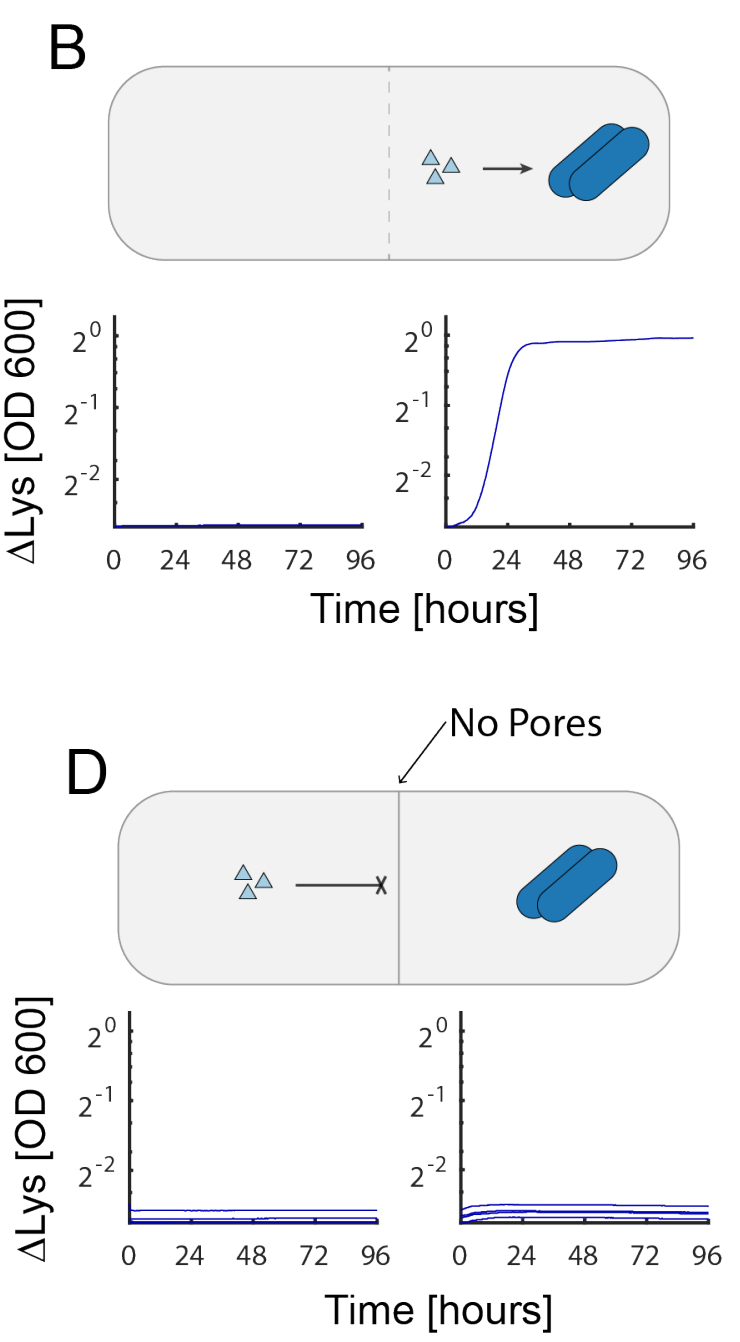

Figure 4: Lysine Diffusion Bioassay with $E$. coli $4 L y s$ in the BioMe Plate

252 Various amino acid diffusion conditions were tested in the BioMe plate. (A) $\Delta$ Lys E. coli

253 auxotrophs grown in minimal media with supplemental lysine across the $0.1 \mu \mathrm{m}$ membrane

254 (n=4). (B) Positive control with lysine supplemented in the same well as the microbial culture

255 ( $\mathrm{n}=1)$. (C) Negative control with no supplemental lysine provided $(\mathrm{n}=1)$. (D) Negative control

256 with supplemental lysine provided across a membrane with no pores $(n=4)$. 
We next co-cultured $\Delta$ Lys and $\Delta$ Ile in the BioMe plate to measure the dynamics of their

258 metabolic interaction (Figure 5). As expected, each strain can complement, to some extent, the

259 amino acid missing in the other, confirming the existence of cross-feeding. Surprisingly,

260 however, the auxotrophs grew significantly slower when inoculated in paired wells separated by

261 a porous membrane than when grown in the same well (Figure 5A and B). The membrane

262 significantly impeded the growth of both members in the syntrophic interaction. As shown in

263 Fig. $5 \mathrm{~A}$, this effect is asymmetric, in that $\Delta$ Ile seems to be able to help $\Delta$ Lys more than $\Delta$ Lys

264 helps $\Delta$ Ile, though the growth of $\Delta$ Ile is still higher than the negative control. Negative controls

265 further confirmed that neither auxotrophs could grow without the partner strain (Figure 5C and

266 D) or when separated by a membrane with no pores (Figure 5E). Qualitatively similar results

267 were observed for membrane pore sizes of $0.03,0.1$, and $0.2 \mu \mathrm{m}$ (Figure S5.1 and S5.2). For

268 membranes of $0.4 \mu \mathrm{m}$ pore size, microbial cross-over through and/or around the membrane/O-

269 ring junction occurred in all replicates (measured by selective plating at the end of the

270 experiment, Supplemental Table 3), preventing any measurement of interaction dynamics. The

271 slow-down of cross-feeding across the membrane (Fig. 5) seems curious, especially in light of

272 the fact that amino acid diffusivity seemed not to be an issue when each organism had the

273 auxotrophic amino acid on the other side of the membrane (Fig. 4). This observation pointed to a

274 subtler concentration-dependent effect, and we wondered whether the quantitative nature of the

275 BioMe experiments could help us gain a deeper understanding of the dynamics underpinning this

276 phenomenon. 


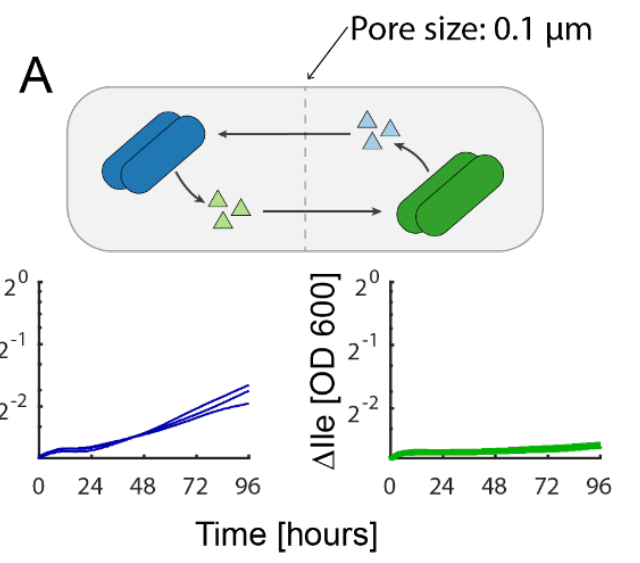

C
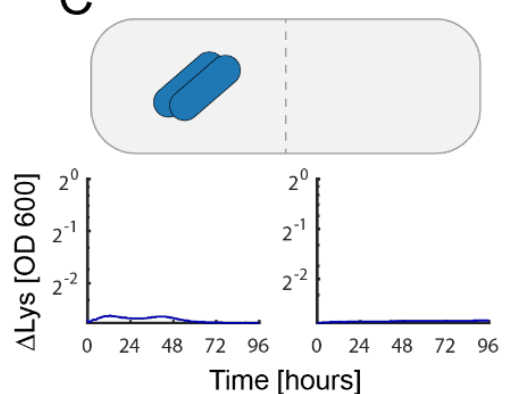

B
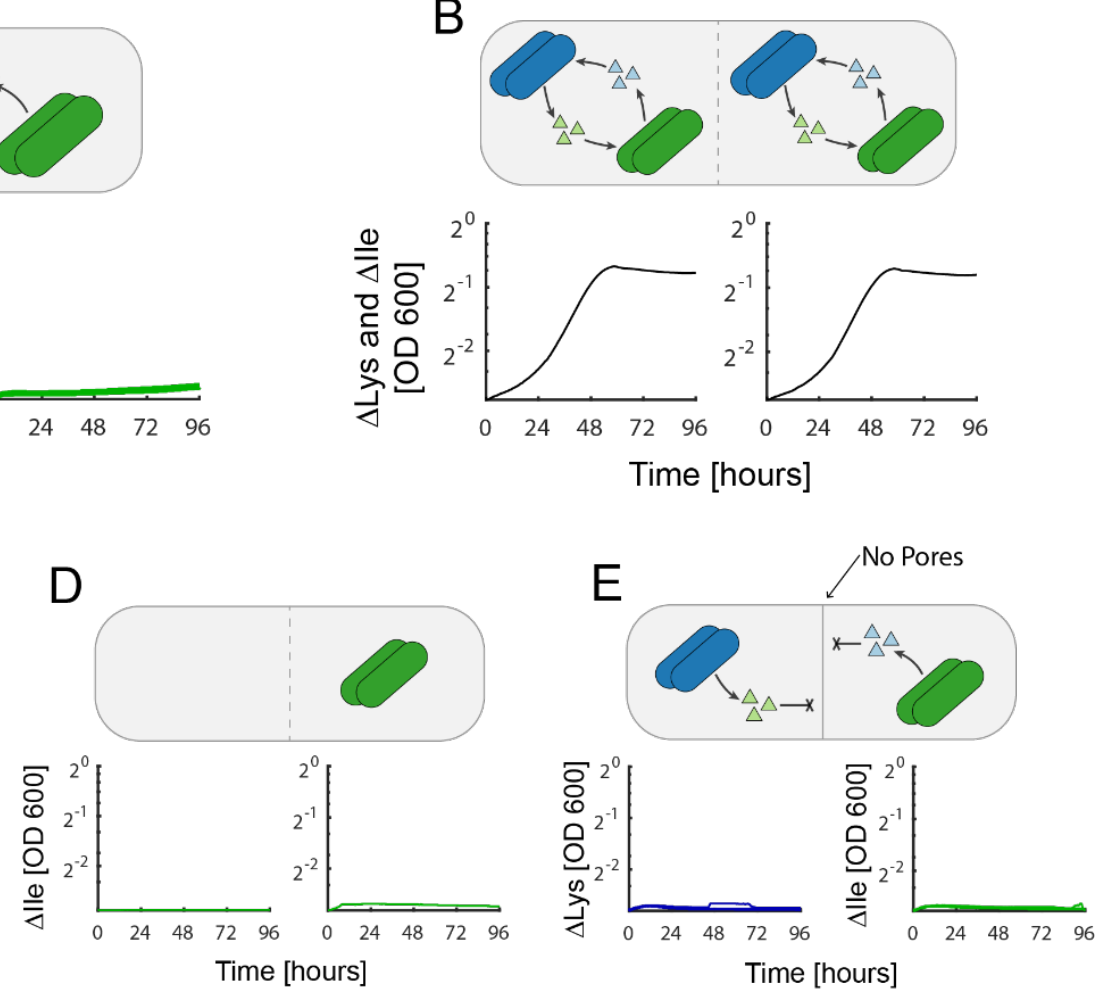

Figure 5: Syntrophic E. coli Lysine and Isoleucine Auxotroph Co-Culture

279 Mutualistic cross-feeding between $\Delta$ Lys and $\Delta$ Ile amino acid auxotrophs. (A) $\Delta$ Lys (blue) and

$280 \Delta$ Ile (green) grown across $0.1 \mu$ m porous membrane $(\mathrm{n}=3)$. (B) Positive control with $\Delta$ Lys and

$281 \Delta$ Ile grown in the same wells $(\mathrm{n}=1)$. (C\&D) Negative controls with $\Delta$ Lys and $\Delta$ Ile without a

282 syntrophic partner, respectively $(\mathrm{n}=1)$. (E) Negative control with $\Delta$ Lys and $\Delta$ Ile separated by a

283 membrane with no pores $(n=3)$. 
286 plate, we developed a computational model describing the syntrophic E. coli auxotroph co-

287 culture experiments. This model captures the dynamics within two interacting wells of the

288 BioMe plate, by simulating the processes of metabolite diffusion across the membrane, glucose

289 and amino acid uptake by the E. coli auxotrophs, and a stoichiometric leakage of the respective

290 partner's auxotrophy amino acids (Figure 6); the full list of equations and parameters is available

291 in supplemental Equations S2 and at https://github.com/segrelab/co_culture_device. Using this

292 computational model, all performed experiments involving the E. coli amino acid auxotrophs can

293 be simulated by changing the initial conditions of the model to match those of the experiment

\section{4 (Figure S6.1).}



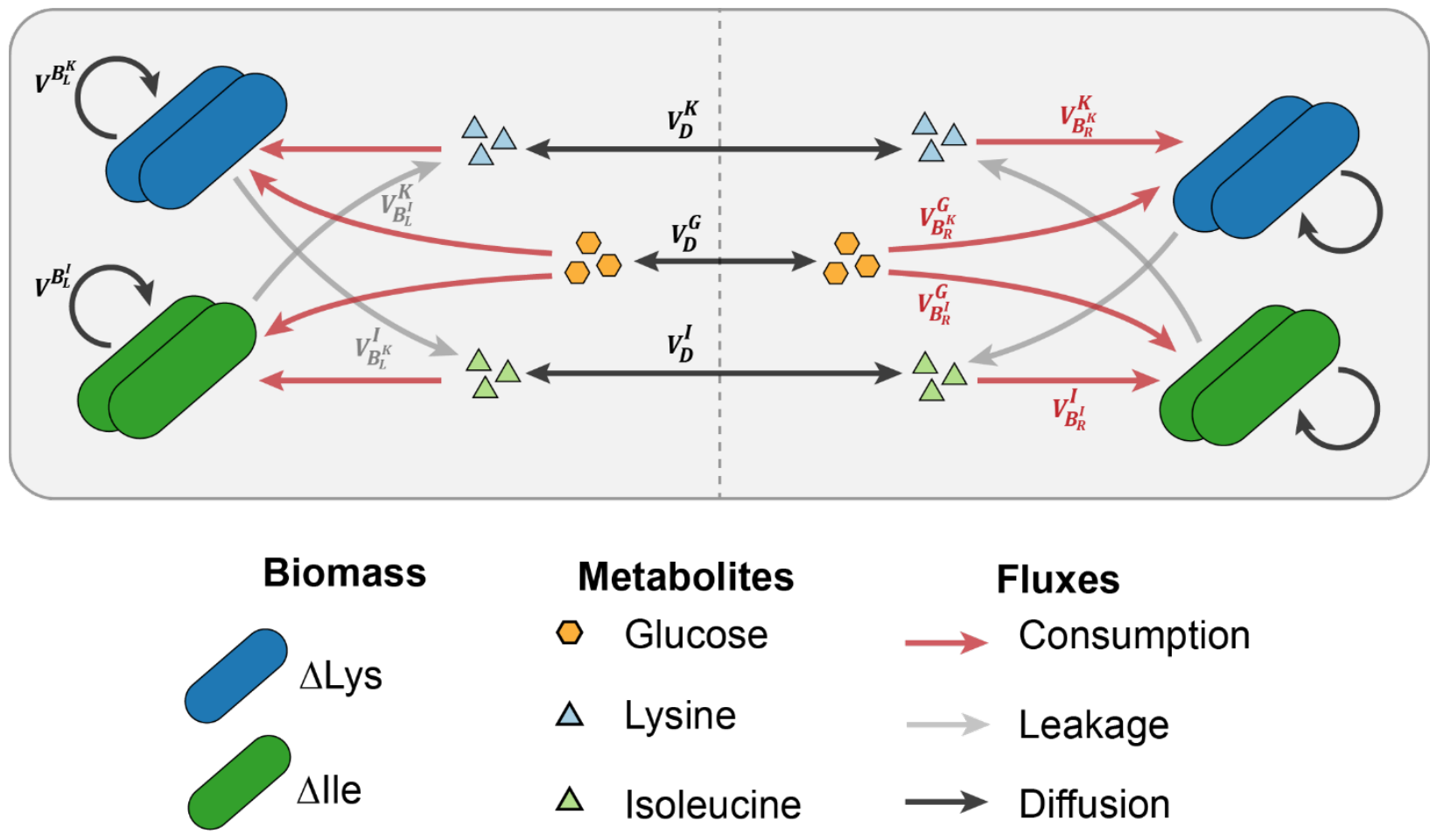

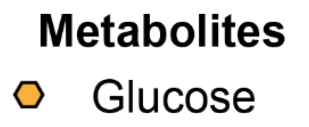

$\Delta$ Lysine

$\Delta$ Isoleucine

\section{Fluxes}

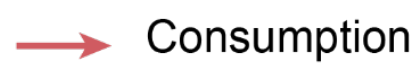

\section{Leakage}

Diffusion

Growth

\section{BioMe}

298 The fluxes of metabolites simulated by the model are illustrated in this figure, including: the

299 diffusion of glucose and amino acids across the porous membrane (black arrows), the uptake of

300 glucose and auxotrophy amino acids supporting growth (red arrows), and the leakage of the

301 respective partner's auxotrophy amino acids (gray arrows). The growth of each auxotroph's

302 biomass is also represented (circular arrow). Supplemental Equations S2 describe all parameters

303 and the full dynamics of the system. 
We integrated this computational model with our syntrophic co-culture experiment to explore the parameter space that gives rise to our primary qualitative observation - that both members of the mutualistically cross-feeding pair grew significantly faster when in the same

307 well than when interacting across the membrane. The parameters for uptake kinetics and biomass

308 stoichiometry were estimated from literature (Supplemental Equations 3). The parameter space

309 was then explored for two important parameters of the interaction, which were difficult to

310 estimate from the literature: the diffusion rate of the metabolites across the membrane and the

311 leakage stoichiometry of the amino acids. In particular, upon varying these parameters, we

312 compared the 48-hours predicted growth yield of an auxotroph grown in the same well with its

313 partner relative to the yield in membrane-separated co-culture (Figure 7A). Distinct regions were

314 clearly visible in this parameter space, which gave rise to different qualitative simulated results

315 (Figure $7 \mathrm{~A}$ and $\mathrm{C}$ ). One can see that if cellular leakage of the amino acid is too small (Figure

316 7A, region 3) the same-well and separate-well simulations behave similarly, i.e. neither supports

317 any growth. If both leakage and diffusion are high enough (Figure 7A, region 1), again the two in

318 silico experiments behave similarly, but this time they both lead to increased yield. Notably there

319 is an intermediate region of the parameter space (Figure 7A, region 2) in which leakage is not a

320 limiting step to guarantee cross-feeding, but diffusion between separate wells is. In this region,

321 the two auxotrophs can secrete enough amino acids to support each other, but diffusion through

322 the BioMe membrane slows down the growth process enough to lead to a detectable difference.

323 The identification of the intermediate region (Fig. 7A, region 2) prompted us explore the

324 possibility of using this modeling framework to provide more insight into the experimentally

325 unknown key parameters. In particular, we used an approximate Bayesian computation approach

326 (see Methods) to infer, and model the uncertainty of, the leakage parameter values that were 
327 consistent with the observed differential growth yields in the same-well positive control versus

328 the membrane-separated co-culture (Figure 7 B). The posterior distribution of the leakage

329 parameter, conditioned on this experimental observation, was estimated by using a statistic of the

330 logarithm of the ratio of the biomass yields at 48 hours (Supplemental Equations S4) and an

331 acceptance cut-off of 2 standard deviations around the experimentally measured mean value (See

332 Methods for further details). To further investigate the asymmetric nature of our observations, a

333 similar analysis was conducted where the lysine and isoleucine leakage parameters were sampled

334 from independent prior distributions, and could thus be inferred separately (Supplemental Figure

335 S7.1). This gave rise to a lysine leakage parameter that was higher than that of isoleucine,

336 consistent with our observation of increased $\Delta$ Lys growth. Furthermore, noise was added to the

337 prior distributions of the literature inferred parameters, by sampling them from a log uniform

338 distribution spanning one order of magnitude around their literature inferred values

339 (Supplemental Figure S7.2 \& S7.3). Even in the presence of this amount of noise in these values,

340 the leakage stoichiometry parameter was effectively constrained by the data. Through this

341 analysis, we integrated novel observations obtained with the BioMe plate with a computational

342 model to infer plausible ranges of the leakage parameter governing the interaction between these

343 auxotrophic E. coli mutants. The posterior distributions obtained from this approximate Bayesian

344 computation approach represent these inferences and their uncertainties, given the assumptions

345 of our model and the data that we conditioned it on. A similar approach could be applied to any

346 observations of interaction dynamics from the BioMe plate to gain insight into how these

347 observations further constrain the inference of different parameters of interest. 


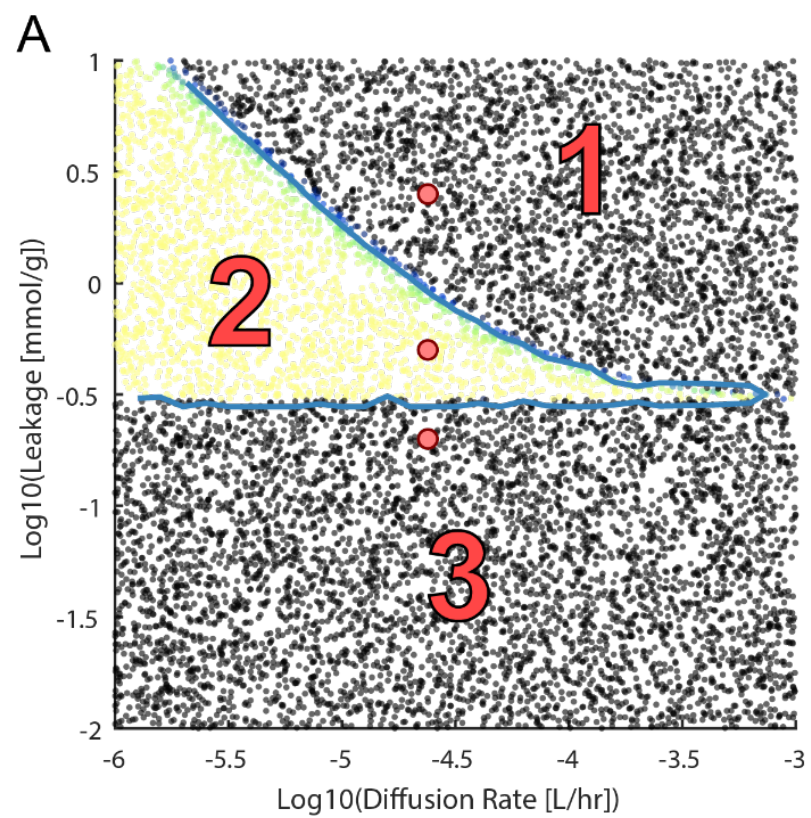

\section{1}
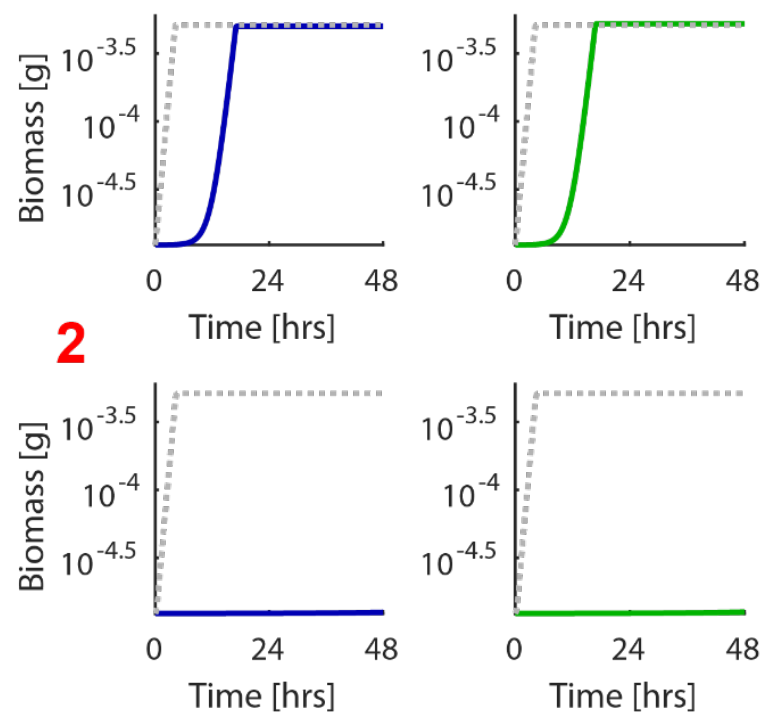
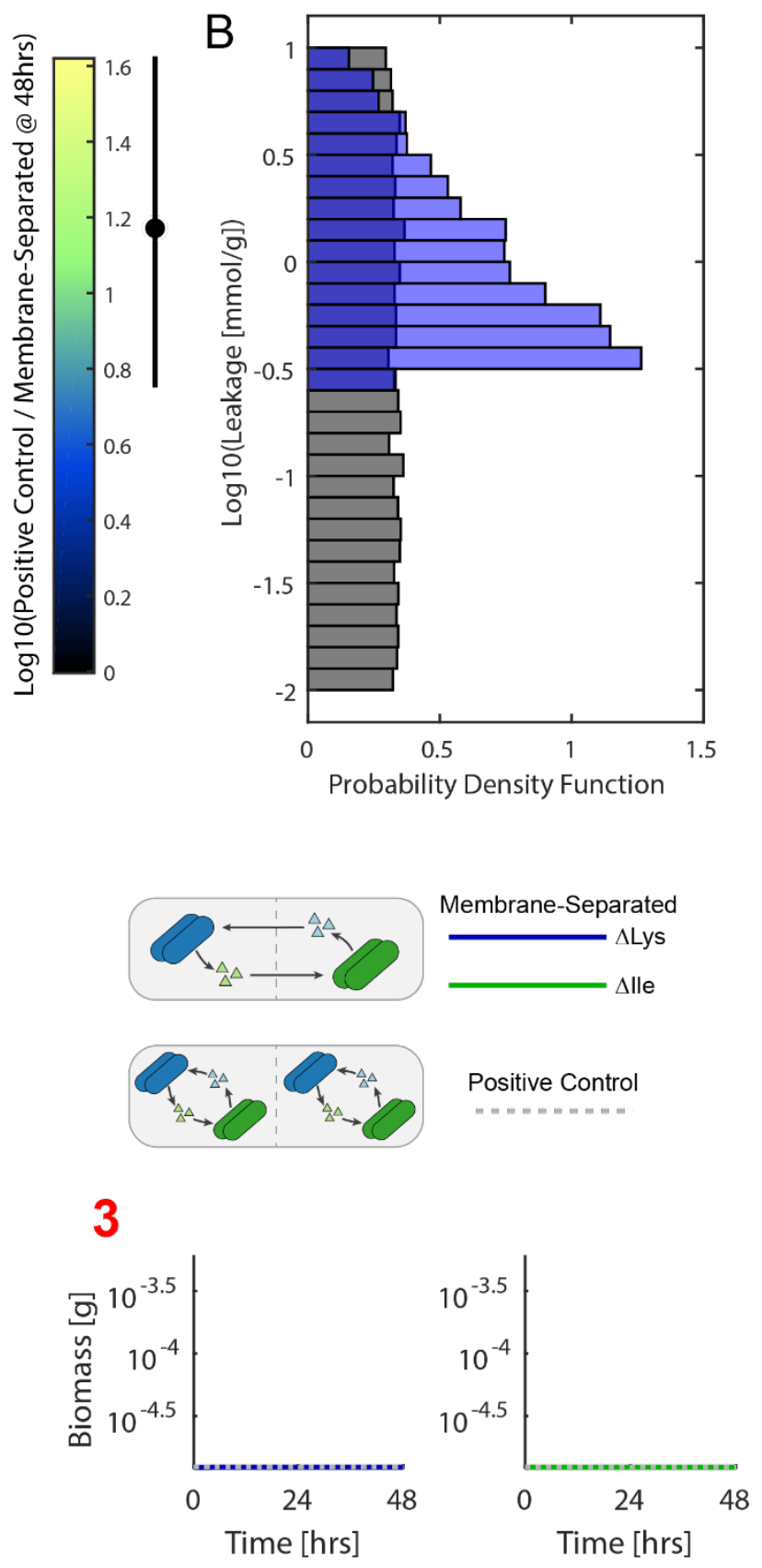

\section{Figure 7: Computational Modeling of $\boldsymbol{E}$. coli auxotroph Co-Culture Interaction Parameters}

350 (A) The sampled space of diffusion and leakage parameters is shown. The diffusion rate and

351 leakage stoichiometry were sampled from log uniform prior distributions. Each point represents

352 a different simulation of the model. The color indicates the log base 10 of the ratio between the

353 yield at 48 hours of $E$. coli auxotrophs grown in the same well (positive control) divided by the 
354 yield in opposite wells (membrane-separated). This value was used as a statistic for approximate

355 Bayesian computation. The experimental average of this statistic (1.18) and two times the

356 standard deviation (0.448) are indicated by the black dot and bar next to the color bar. Points

357 within this range are separated by a blue line outlining region 2 in the scatter plot. (B) The prior

358 (gray) and posterior (blue) distributions of the leakage stoichiometry parameter are plotted as

359 histograms. The prior distribution was sampled from a log uniform distribution. The posterior

360 distribution was calculated by accepting points with a statistic value within 2 standard deviations

361 of the average experimentally observed value. (C) Example growth curves for different regions

362 of the parameter space in A are shown. Simulated growth curves of $\Delta$ Lys (blue line) and $\Delta$ Ile

363 (green line) grown across from each other (membrane-separated), and grown together (gray

364 dotted line, positive control), are shown for three different regions of parameter space. 


\section{Discussion}

We utilized the newly developed BioMe plate to observe a natural symbiotic interaction

367 between organisms found in the D. melanogaster gut microbiome, and to quantitatively study the

368 interaction between two syntrophic engineered E. coli mutants. While our D. melanogaster

369 results demonstrate the utility of BioMe to study a natural microbial interaction between two

370 non-model organisms, corroborating recently published results (38), the better characterized $E$.

371 coli auxotroph interaction allowed us to integrate the data obtained with BioMe into a

372 computational model providing quantitative insight into the parameters of this interaction.

373 Moving forward, as computational modeling of microbial physiology advances, it may be

374 possible to apply such modeling frameworks more ubiquitously. For example, genome-scale

375 metabolic models could be incorporated into a spatial model of the BioMe device to represent

376 the metabolic processes occurring in each interacting population and model emergent

377 interactions (11). Such a model could be used, alongside BioMe observations, to gain

378 quantitative, genome-scale insight into the interactions between microbial organisms, where the

379 mechanism of interaction is not known a priori.

380 Our investigation of the E. coli lysine and isoleucine auxotrophs revealed that their

381 syntrophic interaction was altered by the presence of a porous membrane separating the

382 interacting auxotrophs. These results suggest that certain "high-stakes" interactions could be

383 localized, such that highly-proximal ecological neighbors reap the majority of the benefits from

384 "leaked" communal resources. Leveraging a mechanistic, dynamical model for the syntrophic $E$.

385 coli auxotroph co-culture experiment, we inferred plausible leakage parameters that govern this

386 localized interaction. This result corroborates and complements the recent imaging-based finding

387 that pairs of E. coli proline and tryptophan auxotrophs can best help each other within a small 
local radius around each cell (52). While these local interactions may be dominated by diffusion-mediated processes, we cannot rule out, based on our experiment, that cross-feeding is also facilitated by contact-based mechanisms. Similar phenomena were reported, for example, in

391 bacterial-fungal interactions, which were shown to require physical association for the activation 392 of a cryptic biosynthetic pathway of secondary metabolism in the fungus (53). The local nature

393 of these interactions may have important evolutionary and ecological consequences $(54,55)$, and

394 the BioMe device is a promising platform for the continued study of such phenomena. It is

395 conceivable that analyses similar to the one preformed here for the E. coli auxotrophs, could be

396 extended in the future to the study of natural interaction partners, such as the D. melanogaster

397 gut microbiome strains $(38,50,51)$, or other pairs of strains suspected to interact with each other

398 through the exchange of metabolites.

There are some key limitations to the use of the BioMe plate. First, as is clear from our

400 results, the porous membranes can have a significant impact on mutualistic cross-feeding

401 interactions. In addition to altering the dynamics of metabolite exchange, the membrane limits

402 interactions to only those mediated by diffusible molecules. Despite placing a constraint on the

403 type of interactions that can be investigated, this limitation can be beneficial for the quantitative

404 study and design of microbial consortia. For example, limiting interactions to those mediated by

405 diffusible molecules makes it easier to robustly integrate and compare experimental

406 measurements with metabolic models. Another limitation of the BioMe device is the fact that

407 microbial cross-over can occur across the membranes. In our syntrophic auxotrophs co-culture

408 experiment we observed microbial cross-over in $2 / 9$ pairwise assays with $0.03,0.1$, and $0.2 \mu \mathrm{m}$

409 pore sizes, and $3 / 3$ for the $0.4 \mu \mathrm{m}$ pore size. These studies were exquisitely sensitive for detecting

410 microbial cross-over as the mutualistic interaction between the two strains would effectively 
411 amplify any crossed-over organisms. Future studies could focus on more thoroughly

412 characterizing the cross-over process for a variety of different organisms and conditions. For

413 studies aimed at observing microbial interactions, the choice of membrane pore size should be

414 carefully considered to restrict microbial cross-over and simultaneously allow for desired

415 diffusion of metabolites.

416 Regarding the manufacturing of the BioMe device, it should be noted that while the body

417 segments can be fabricated using either milling or 3D printing methods, there is a significant

418 trade-off between precision and flexibility in these two methods. We found that devices

419 fabricated using milling had finer tolerances and were thus less prone to leakage than those that

420 were 3D printed. However, the 3D printing approach facilitates rapid and easy prototyping of

421 alternative plate layouts. Ultimately, users should choose the fabrication technique that best suits 422 their goals.

423 Regarding the pore size dependence of small-molecule diffusion in the device, there was

424 an interesting observation in the chemical dye diffusion experiments - the estimated diffusion

425 constant was consistently higher when calculated using data from the input well where the dye

426 was initially placed as opposed to that of the opposite well. This effect was more pronounced for

427 the larger pore sizes and for phenol red. A possible explanation for this effect is that the dye is

428 settling or being sequestered in the membrane/device, and thus appears to be diffusing faster

429 from the initial well and more slowly into the opposite well. This effect was not included in

430 subsequent modeling efforts, as it produced only minor differences in the inferred diffusion

431 constant relative to the range of uncertainty in the diffusion parameter considered in our model.

432 Although we demonstrate here the use of the BioMe plate to measure pairwise

433 interactions, the device is a flexible technology that could be re-designed to incorporate multi- 
434 member assays and higher-order interactions. 3D printing accessibility enables rapid fabrication

435 and validation of potential plate layouts. Going beyond pairwise interactions, selected consortia

436 of microbial organisms could be grown in each well of the BioMe plate to facilitate the

437 investigation of higher-order interactions, which are thought to have an important impact on

438 microbial community structure (56-58). Entire microbial communities could similarly be grown

439 across from individual isolates to observe their growth supporting capabilities, as done with the

440 iChip device (49), but with the added capability of observing growth dynamics. Furthermore, the

441 3D-printed design of the BioMe body segments could be modified to design novel interaction

442 chambers, such as multiple wells connected to a central mixing chamber or sequential wells

443 connected by porous membranes. Overall, the BioMe plate is relatively easy to manufacture and

444 implement, and the use of this device to study the interaction of microbial organisms from a

445 multitude of different contexts will help improve our understanding of and ability to engineer

446 microbial communities. 


\section{Methods}

\section{BioMe Fabrication}

All materials required for BioMe fabrication are detailed in Supplemental Table 1. O-

450 rings, 6-32 flange nuts. 4-40 screws, and membranes were bought ready to use. 6" 6-32 rods

451 were cut to $125 \mathrm{~mm}$ with a grind wheel. Stock food-grade silicone rubber sheets were laser-cut to

452 gasket specifications using an Epilog Laser Mini 60W laser cutter; wrapping in dampened shop

453 towels helped mitigate charring. The transparent base was CNC milled from clear polycarbonate

454 sheets.

455 The body segments could either be machined or 3D-printed. Two separate CNC milling

456 operations were required to machine the vertical and horizontal features of the body segment

457 from polypropylene sheet. A drill press was used to complete the spot holes, with each screw

458 hole manually tapped with a 4-40 tap bit, and each body segment deburred and washed.

459 Alternatively, stereolithography (SLA) resin 3D-printing can be used to fabricate the body

460 segments. FormLabs' Form2 SLA printers were used with the Dental SG Resin, a biocompatible,

461 autoclavable resin. Screw holes were manually tapped and body segments were sanded to size.

\section{Leakage Test}

463 A simple visualization test was used to test water-tight seal throughout the assembled

464 BioMe plate. All wells were loaded with $250 \mu \mathrm{L}$ of $100 \mu \mathrm{M}$ phenol red. The core BioMe device

465 was then placed atop a paper towel and fit into the bottom tray and covered with the top lid. The

466 BioMe plate was left in the shaking incubator overnight and no leakage was verified. 
467

468

469

470

471

472

473

474

475

476

477

478

\section{Sterilization Protocol and Validation}

To sterilize the BioMe plate after use, the device is disassembled, membranes disposed, components dishwashed, and then autoclaved (Gravity, 30mins exposure/15mins dry). For sterile assembly, a biosafety cabinet is recommended. Membranes are bathed in $70 \%$ ethanol for 30mins. Pre-sterilized components are then sequentially assembled with the membranes in sterile conditions. For additional caution, pre-sterilized device components may be ethanol bathed prior to assembly.

Sterilization protocol was validated to ensure no post-contamination. A sterilized reassembled BioMe plate was loaded with $250 \mu \mathrm{L}$ LB Miller media $(10 \mathrm{~g} / \mathrm{L}$ tryptone, $10 \mathrm{~g} / \mathrm{L} \mathrm{NaCl}$, $5 \mathrm{~g} / \mathrm{L}$ yeast extract) per well and placed into a $30^{\circ} \mathrm{C}$ static incubator for $72 \mathrm{hrs}$. A $10 \mu \mathrm{L}$ aliquot from each well was then plated onto LB agar plates. Plates were then incubated at $30^{\circ} \mathrm{C}$ for 72hrs. No microbial growth was confirmed.

\section{Colorimetric Dye Diffusion}

Calibration curves for phenol red (PR) and bromocresol purple (BP) were determined to relate optical density at isosbestic point (478nm for PR and 490nm for BP) to concentration. A BioMe plate was assembled with membranes with no pores. Columns were loaded with $250 \mu \mathrm{L}$ of varying concentrations of dye: $450,400,350,300,250,200,150,100,50,0 \mu \mathrm{M}$; top three

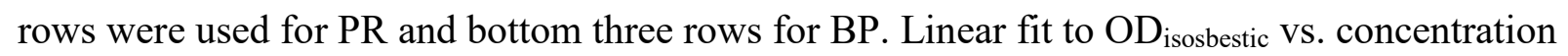
data points used for calibration curve.

Diffusion experiments were used to estimate the diffusion coefficients of various membrane pore sizes for PR and BP. A BioMe plate was assembled with varying membrane pore sizes at each body junction: no pores, $0.03,0.1,0.2$, and $0.4 \mu \mathrm{m} .250 \mu \mathrm{L}$ of $400 \mu \mathrm{M}$ dye was 
loaded into the left-column, and $250 \mu \mathrm{L}$ of distilled water in the right-column for each pore size.

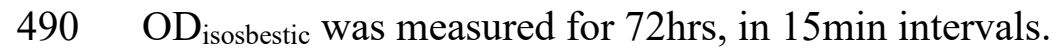

Diffusion constants were estimated using a gradient-driven diffusion model, provided in

492 supplemental Equations S1. The time dependent value on the left hand side of supplemental

493 Equations S1 was calculated from the data and fit to an exponential function (using MATLAB

494 function fit with fittype: exp1) to infer our model's diffusion constant for both dyes and pore

495 sizes of $0.03,0.1,0.2$, and $0.4 \mu \mathrm{m}$. The units for the diffusion constant, $d$, in our equations are

$496 \mathrm{~L} / \mathrm{hr}$ such that the flux has units of mmol/hr. Our model is similar to Fick's first law, in that there

497 is a gradient driven diffusion, however in our system the diffusion area and distance are fixed

498 and thus lumped into the diffusion parameter giving rise to units that are different from a

499 traditional "diffusion constant".

500 Drosophila Gut Microbiome Interaction

$501 \quad$ Strains of Acetobacter oryzifermentans, Lactobacillus brevis, and Lactobacillus

502 plantarum were isolated as previously described (59) and streaked onto YPD agar plates (10g/L

503 peptone, $10 \mathrm{~g} / \mathrm{L}$ yeast extract, $8 \mathrm{~g} / \mathrm{L}$ dextrose, $15 \mathrm{~g} / \mathrm{L}$ agar). Identity and lack of cross-

504 contamination were confirmed by colony PCR using species-specific primers and gel

505 electrophoresis (43). For each strain, four clonal replicates were picked from colonies, and grown

506 in $5 \mathrm{~mL}$ of YPD broth $(10 \mathrm{~g} / \mathrm{L}$ peptone, $10 \mathrm{~g} / \mathrm{L}$ yeast extract, $8 \mathrm{~g} / \mathrm{L}$ dextrose $)$ at $30^{\circ} \mathrm{C}$ in a static

507 incubator for 20hrs. Cells were centrifuged and pellets washed thrice in PBS. OD600 of $250 \mu \mathrm{L}$

508 of each culture and PBS blanks were read. Cultures were diluted with YPD media to an

509 OD600 $=0.1$ using following formula:

510

$$
\% \text { Culture in Dilution }=\frac{\text { Set } O D 600-P B S O D 600}{\text { Culture } O D 600-P B S O D 600}=\frac{0.1-P B S}{C-P B S}
$$


511 Dilutions were re-done for those not within 10\% deviation. Cultures were further diluted 1:100

512 in YPD media. Assembled BioMe plate, with $0.03 \mu \mathrm{m}$ pores size membranes, was loaded with

513 diluted cultures in appropriate wells and sealed with parafilm. The plate was then run on a plate-

514 reader for $96 \mathrm{hrs}, 30^{\circ} \mathrm{C}$, with OD600 measurements at $15 \mathrm{mins}$ intervals.

\section{Syntrophic Co-Culture Interaction}

516 Diffusion of amino acids through membranes of various pore sizes was validated using

517 Lambda Red-recombineered EcNR1 E. coli for knock-out of lysA ( $\Delta$ Lys) and ilvA $(\Delta \mathrm{Ile})(44)$.

518 Strains were streaked and selected on LB+Cam $\left(25 \frac{\mu \mathrm{g}}{\mathrm{mL}}\right.$ chloramphenicol $)$ agar plates. Strains were 519 grown in $5 \mathrm{~mL}$ of $\mathrm{LB}+\mathrm{Cam}$ broth $(\mathrm{n}=4$ for both $\Delta$ Lys and $\Delta \mathrm{Ile})$ in a 12 -well plate for $24 \mathrm{hr}$ inside

520 the plate-reader, with OD600 measurements every 15mins. These growth curves were used to

521 determine dilution time $\left(\mathrm{t}_{\mathrm{dil}}=6 \mathrm{hrs}\right)$ for proceeding experiments (Supplemental Figure S4.4).

$522 \quad$ For amino acid diffusion experiment, a BioMe plate was assembled with varying

523 membrane pore sizes at each body junction: no pores, $0.03,0.1,0.2$, and $0.4 \mu \mathrm{m}$. For either strain,

524 six clonal replicates were grown in $5 \mathrm{~mL}$ of $\mathrm{LB}+\mathrm{Cam}$ broth at $30^{\circ} \mathrm{C}$ in a static incubator for $6 \mathrm{hrs}$,

525 and then washed in minimal media (M9 + Glucose $(0.4 \%)+$ Thiamine $\left(1 \frac{\mu g}{m L}\right)+$ Biotin $\left(1 \frac{\mu g}{m L}\right)+$

526 chloramphenicol $\left.\left(25 \frac{\mu g}{m L}\right)\right)$. Cultures were diluted with minimal media to an OD600 $=0.1$ using

527 formula provided in Drosophila Gut Microbiome Interaction methods. Cultures were further

528 diluted 1:100 in both minimal media and minimal media supplemented with lysine $(0.0134 \%)$ or

529 isoleucine $(0.0065 \%)$, for $\Delta$ Lys or $\Delta$ Ile, respectively. A sterile assembled BioMe plate was

530 loaded with diluted cultures in appropriate wells - for each pore size, $\mathrm{n}=4$ for amino acid

531 diffusion assay, $\mathrm{n}=1$ for negative control with no supplemental amino acid, and $\mathrm{n}=1$ for positive 
532 control with auxotroph loaded with supplemental amino acid in same well. BioMe plate was run

533 on the plate-reader for $96 \mathrm{hrs}, 30^{\circ} \mathrm{C}$, with OD600 measurements at $15 \mathrm{mins}$ intervals.

534 Similar growth, wash, and dilution procedure was followed for the syntrophic co-culture

535 experiment. For each pore size, $n=3$ for co-culture assay across membrane, $n=1$ for both negative

536 controls without co-culture partner, and $n=1$ for positive control with co-culture partners in same

537 well. Plate-reader was run with BioMe plate for $96 \mathrm{hrs}, 30^{\circ} \mathrm{C}$, with OD600 measurements at

$53815 \mathrm{mins}$ intervals. At end of kinetic read, a $5 \mu \mathrm{L}$ aliquot from each well was cultured on minimal

539 media agar plates supplemented with either lysine or isoleucine to determine instances of cross-

540 over. Plates were cultured for $72 \mathrm{hrs}$ at $30^{\circ} \mathrm{C}$ in a static incubator.

\section{$541 \quad$ Computational Modeling}

We developed a computational model to simulate the interaction between $E$. coli amino

543 acid auxotrophs in the BioMe device. Metabolite diffusion is modeled by a gradient driven flux

544 across the porous membrane. Metabolite uptake flux is bounded by Michaelis-Menten kinetic

545 uptake equations, and growth rate is determined by the minimum biomass flux that can be

546 produced based on metabolite uptake rates and biomass stoichiometry. Amino acid leakage is

547 determined by a stoichiometric parameter specifying the amount of amino acid leaked into the

548 environment for each gram of E. coli biomass produced. The uptake, growth constraints, and

549 leakage components of this model are analogous to previously developed dynamic flux balance

550 analysis models (11). Thus our model is essentially a simplified dynamic flux balance analysis

551 model with a growth rate proportional leakage term and gradient driven diffusion between

552 compartments. The full model dynamics are presented in supplemental Equations S2 and the

553 code is available at https://github.com/segrelab/co_culture_device. Simulation of the model was

554 implemented in MATLAB. Testing of run-time and accuracy was conducted to benchmark three 
MATLAB ODE solvers (ode23tb, ode45, and ode15s). The function ode23tb was found to have

556 the best performance, with consistent accuracy when using maximum step sizes of $0.0025 \mathrm{hrs}$.

557 Thus, this solver function and maximum step size setting was used to simulate model dynamics.

We implemented an approximate Bayesian computation based approach to integrate

560 infer the posterior distribution of the parameters of a computational model by using simulations

561 of experimental results to approximate the likelihood of the data $(60,61)$. We implemented a

562 rejection based algorithm where sample parameters are initially drawn from a given prior

563 distribution and are included in the posterior distribution if the difference between the simulated

564 and experimental value of a given statistic is less than some specified threshold. The statistic that

565 we utilized was the $\log$ base 10 of the ratio between growth at 48 hours of the $E$. coli auxotrophs

566 in the same well (positive control) divided by the growth in opposite wells (membrane-

567 separated) (Supplemental Equations 4). Using this ratio allowed us to compare experimental

568 results to simulated results without calibrating between biomass units, as our optical density

569 growth measurements were made ratio-scale by subtracting a blank control. The threshold that

570 we used was two standard deviations from the experimentally measured mean ratio.

571 We began by sampling diffusion and leakage parameters while assuming that the leakage

572 of isoleucine and lysine were equal (Figure 7). The uptake kinetics and biomass parameters were

573 estimated from the literature and were fixed at their estimated values (prior distribution was a

574 Dirac delta function) (Supplemental Equations S3). The leakage and diffusion parameters were

575 randomly sampled from a log uniform prior distribution ranging from $10^{-6}$ to $10^{-3} \mathrm{~L} / \mathrm{hr}$ for the

576 diffusion rate and $10^{-2}$ to $10 \mathrm{mmol} / \mathrm{g}$ for the leakage stoichiometry. Next, we used our model to

577 investigate the unequal growth of the $\Delta$ Lys and $\Delta$ Ile auxotrophs (Figure S7.1). We fixed the 
578 diffusion rate to a value on the high-end of what we observed for the diffusion of the chemical

579 dyes $\left(3.68 * 10^{-5} \mathrm{~L} / \mathrm{hr}\right)$. Then, sampled the leakage for $\Delta$ Lys and $\Delta$ Ile independently from log

580 uniform prior distributions with the same ranges as used previously. Finally, we added noise to

581 all of the literature estimated parameters (with the exception of volume) to represent an increased

582 level of uncertainty (Figure S7.2 and S7.3). We sampled all literature estimated parameters from

583 a log uniform prior distribution that varied by 1 order of magnitude around the originally fixed

584 literature estimated value. This more uncertain prior distribution was used to repeat the inference

585 of leakage stoichiometry for both the equal (Figure S7.2) and unequal (Figure S7.3) cases.

\section{Data Availability}

587 All data and code are available on GitHub at https://github.com/segrelab/co culture device. Data

588 includes raw kinetic optical density measurements for the small molecule dye diffusion,

589 Drosophila gut microbe co-culture, amino acid diffusion, and syntrophic E. coli auxotroph co-

590 culture experiments, and MATLAB scripts for generation of all figures from raw data are

591 provided in the "raw_data_and_plots" subdirectory. MATLAB scripts for solving the differential

592 equations of the computational model and for the Bayesian analyses are located in the

593 "modeling” subdirectory. 


\section{Acknowledgements}

595 We are grateful to members of the Segrè lab for helpful feedback and constructive advice, and to

596 Harris Wang and Ali Zomorrodi for making the E. coli auxotroph strains available to us.

597 Specifically, we would like to thank Melisa Osborne for assistance with experimental protocols

598 and training. We would also like to acknowledge the staff of the Boston University Engineering

599 Product Innovation Center and Jonathan Perry of the Biology Department Workshop for

600 assistance in the initial fabrication of the device. This work was partially supported by grants

601 from the National Institutes of Health (National Institute of General Medical Sciences, award

602 R01GM121950; National Institute of Dental and Craniofacial Research, award number

603 R01DE024468), the National Science Foundation (grants 1457695 and NSFOCE-BSF 1635070),

604 the Human Frontiers Science Program (RGP0020/2016), and the Boston University

605 Interdisciplinary Biomedical Research Office. DBB acknowledges support by the Boston

606 University training program in quantitative biology and physiology under Ruth L Kirschstein

607 National Research Service Award T32GM008764 from the National Institute of General Medical

608 Sciences. 


\section{References}

610 1. Ponomarova O, Patil KR. 2015. Metabolic interactions in microbial communities: untangling the Gordian knot. Current Opinion in Microbiology 27:37-44.

2. Pacheco AR, Segrè D. 2019. A multidimensional perspective on microbial interactions. FEMS Microbiol Lett 366.

3. Faust K, Raes J. 2012. Microbial interactions: from networks to models. 8. Nature Reviews Microbiology 10:538-550.

4. Goldford JE, Lu N, Bajić D, Estrela S, Tikhonov M, Sanchez-Gorostiaga A, Segrè D, Mehta P, Sanchez A. 2018. Emergent simplicity in microbial community assembly. Science 361:469-474.

5. Friedman J, Higgins LM, Gore J. 2017. Community structure follows simple assembly rules in microbial microcosms. 5. Nature Ecology \& Evolution 1:1-7.

6. Hayes CS, Aoki SK, Low DA. 2010. Bacterial Contact-Dependent Delivery Systems. Annu Rev Genet 44:71-90.

7. Pande S, Shitut S, Freund L, Westermann M, Bertels F, Colesie C, Bischofs IB, Kost C. 2015. Metabolic cross-feeding via intercellular nanotubes among bacteria. 1. Nature Communications 6:6238.

8. García-Bayona L, Comstock LE. 2018. Bacterial antagonism in host-associated microbial communities. Science 361.

9. Koraimann G, Wagner MA. 2014. Social behavior and decision making in bacterial conjugation. Front Cell Infect Microbiol 4.

10. Phelan VV, Liu W-T, Pogliano K, Dorrestein PC. 2012. Microbial metabolic exchangethe chemotype-to-phenotype link. 1. Nature Chemical Biology 8:26-35.

11. Harcombe WR, Riehl WJ, Dukovski I, Granger BR, Betts A, Lang AH, Bonilla G, Kar A, Leiby N, Mehta P, Marx CJ, Segrè D. 2014. Metabolic resource allocation in individual microbes determines ecosystem interactions and spatial dynamics. Cell Rep 7:1104-1115.

12. Granato ET, Meiller-Legrand TA, Foster KR. 2019. The Evolution and Ecology of Bacterial Warfare. Current Biology 29:R521-R537.

13. Miller MB, Bassler BL. 2001. Quorum Sensing in Bacteria. Annu Rev Microbiol 55:165199.

14. Ratzke C, Gore J. 2018. Modifying and reacting to the environmental $\mathrm{pH}$ can drive bacterial interactions. PLOS Biology 16:e2004248.

15. Embree M, Liu JK, Al-Bassam MM, Zengler K. 2015. Networks of energetic and metabolic interactions define dynamics in microbial communities. PNAS 112:15450-15455.

16. D'Souza G, Shitut S, Preussger D, Yousif G, Waschina S, Kost C. 2018. Ecology and evolution of metabolic cross-feeding interactions in bacteria. Nat Prod Rep 35:455-488.

17. Mee MT, Wang HH. 2012. Engineering ecosystems and synthetic ecologies. Mol BioSyst 8:2470-2483.

18. Gupta S, Ross TD, Gomez MM, Grant JL, Romero PA, Venturelli OS. 2020. Investigating the dynamics of microbial consortia in spatially structured environments. 1. Nature Communications 11:2418.

19. Antoniewicz MR. 2020. A guide to deciphering microbial interactions and metabolic fluxes in microbiome communities. Current Opinion in Biotechnology 64:230-237.

20. Fisher CK, Mehta P. 2014. Identifying Keystone Species in the Human Gut Microbiome from Metagenomic Timeseries using Sparse Linear Regression. PLoS ONE 9:e102451. 
21. Welch JLM, Rossetti BJ, Rieken CW, Dewhirst FE, Borisy GG. 2016. Biogeography of a human oral microbiome at the micron scale. PNAS 113:E791-E800.

22. Cordero OX, Wildschutte H, Kirkup B, Proehl S, Ngo L, Hussain F, Le Roux F, Mincer T, Polz MF. 2012. Ecological populations of bacteria act as socially cohesive units of antibiotic production and resistance. Science 337:1228-1231.

23. Long RA, Azam F. 2001. Antagonistic Interactions among Marine Pelagic Bacteria. Appl Environ Microbiol 67:4975-4983.

24. Burkholder PR, Pfister RM, Leitz FH. 1966. Production of a Pyrrole Antibiotic by a Marine Bacterium1. Appl Microbiol 14:649-653.

25. Vetsigian K, Jajoo R, Kishony R. 2011. Structure and Evolution of Streptomyces Interaction Networks in Soil and In Silico. PLOS Biology 9:e1001184.

26. Van Rossum T, Ferretti P, Maistrenko OM, Bork P. 2020. Diversity within species: interpreting strains in microbiomes. Nature Reviews Microbiology 1-16.

27. Bharti R, Grimm DG. 2019. Current challenges and best-practice protocols for microbiome analysis. Briefings in Bioinformatics bbz155.

28. Goers L, Freemont P, Polizzi KM. 2014. Co-culture systems and technologies: taking synthetic biology to the next level. J R Soc Interface 11.

29. Kehe J, Kulesa A, Ortiz A, Ackerman CM, Thakku SG, Sellers D, Kuehn S, Gore J, Friedman J, Blainey PC. 2019. Massively parallel screening of synthetic microbial communities. PNAS 116:12804-12809.

30. Ge Z, Girguis PR, Buie CR. 2016. Nanoporous microscale microbial incubators. Lab Chip 16:480-488.

31. Hsu RH, Clark RL, Tan JW, Ahn JC, Gupta S, Romero PA, Venturelli OS. 2019. Microbial Interaction Network Inference in Microfluidic Droplets. Cell Systems 9:229-242.e4.

32. Park J, Kerner A, Burns MA, Lin XN. 2011. Microdroplet-Enabled Highly Parallel CoCultivation of Microbial Communities. PLOS ONE 6:e17019.

33. Burmeister A, Grünberger A. 2020. Microfluidic cultivation and analysis tools for interaction studies of microbial co-cultures. Current Opinion in Biotechnology 62:106-115.

34. Moutinho TJ, Panagides JC, Biggs MB, Medlock GL, Kolling GL, Papin JA. 2017. Novel co-culture plate enables growth dynamic-based assessment of contact-independent microbial interactions. PLOS ONE 12:e0182163.

35. Nichols D, Cahoon N, Trakhtenberg EM, Pham L, Mehta A, Belanger A, Kanigan T, Lewis K, Epstein SS. 2010. Use of Ichip for High-Throughput In Situ Cultivation of "Uncultivable" Microbial Species. Appl Environ Microbiol 76:2445-2450.

36. Bollmann A, Lewis K, Epstein SS. 2007. Incubation of Environmental Samples in a Diffusion Chamber Increases the Diversity of Recovered Isolates. Appl Environ Microbiol 73:6386-6390.

37. Sher D, Thompson JW, Kashtan N, Croal L, Chisholm SW. 2011. Response of Prochlorococcus ecotypes to co-culture with diverse marine bacteria. 7. The ISME Journal 5:1125-1132.

38. Sommer AJ, Newell PD. 2019. Metabolic Basis for Mutualism between Gut Bacteria and Its Impact on the Drosophila melanogaster Host. Appl Environ Microbiol 85.

39. Buchon N, Broderick NA, Lemaitre B. 2013. Gut homeostasis in a microbial world: insights from Drosophila melanogaster. 9. Nature Reviews Microbiology 11:615-626. 
698

40. Lee W-J, Brey PT. 2013. How Microbiomes Influence Metazoan Development:Insights from History and Drosophila Modeling of Gut-Microbe Interactions. Annual Review of Cell and Developmental Biology 29:571-592.

41. Fischer CN, Trautman EP, Crawford JM, Stabb EV, Handelsman J, Broderick NA. 2017. Metabolite exchange between microbiome members produces compounds that influence Drosophila behavior. eLife 6:e18855.

42. Adair KL, Wilson M, Bost A, Douglas AE. 2018. Microbial community assembly in wild populations of the fruit fly Drosophila melanogaster. 4. The ISME Journal 12:959-972.

43. Simhadri RK. 2018. Role of Wolbachia in shaping the microbiome of Drosophila melanogaster.

44. Mee MT, Collins JJ, Church GM, Wang HH. 2014. Syntrophic exchange in synthetic microbial communities. PNAS 111:E2149-E2156.

45. Wintermute EH, Silver PA. 2010. Emergent cooperation in microbial metabolism. Molecular Systems Biology https://doi.org/10.1038/msb.2010.66.

46. Shou W, Ram S, Vilar JMG. 2007. Synthetic cooperation in engineered yeast populations. Proc Natl Acad Sci USA 104:1877-1882.

47. Williams CH, David Arscott L, Matthews RG, Thorpe C, Wilkinson KD. 1979. [37] Methodology employed for anaerobic spectrophotometric titrations and for computerassisted data analysis, p. 185-198. In Methods in Enzymology. Academic Press.

48. El-Ashgar NM, El-Basioni AI, El-Nahhal IM, Zourob SM, El-Agez TM, Taya SA. 2012. Sol-Gel Thin Films Immobilized with Bromocresol Purple pH-Sensitive Indicator in Presence of Surfactants. ISRN Analytical Chemistry. Research Article, Hindawi.

49. Berdy B, Spoering AL, Ling LL, Epstein SS. 2017. In situ cultivation of previously uncultivable microorganisms using the ichip. 10. Nature Protocols 12:2232-2242.

50. Consuegra J, Grenier T, Akherraz H, Rahioui I, Gervais H, da Silva P, Leulier F. 2020. Metabolic Cooperation among Commensal Bacteria Supports Drosophila Juvenile Growth under Nutritional Stress. iScience 23:101232.

51. Pais IS, Valente RS, Sporniak M, Teixeira L. 2018. Drosophila melanogaster establishes a species-specific mutualistic interaction with stable gut-colonizing bacteria. PLoS Biol 16:e2005710.

52. Dal Co A, van Vliet S, Kiviet DJ, Schlegel S, Ackermann M. 2020. Short-range interactions govern the dynamics and functions of microbial communities. 3. Nature Ecology \& Evolution 4:366-375.

53. Schroeckh V, Scherlach K, Nützmann H-W, Shelest E, Schmidt-Heck W, Schuemann J, Martin K, Hertweck C, Brakhage AA. 2009. Intimate bacterial-fungal interaction triggers biosynthesis of archetypal polyketides in Aspergillus nidulans. PNAS 106:14558-14563.

54. Stump SM, Johnson EC, Klausmeier CA. 2018. Local interactions and self-organized spatial patterns stabilize microbial cross-feeding against cheaters. Journal of The Royal Society Interface 15:20170822.

55. Cordero OX, Datta MS. 2016. Microbial interactions and community assembly at microscales. Current Opinion in Microbiology 31:227-234.

56. Bairey E, Kelsic ED, Kishony R. 2016. High-order species interactions shape ecosystem diversity. 1. Nature Communications 7:12285.

57. Sanchez-Gorostiaga A, Bajić D, Osborne ML, Poyatos JF, Sanchez A. 2019. High-order interactions distort the functional landscape of microbial consortia. PLOS Biology 17:e3000550. 
744 58. Mickalide H, Kuehn S. 2019. Higher-Order Interaction between Species Inhibits Bacterial Invasion of a Phototroph-Predator Microbial Community. cels 9:521-533.e10.

59. Simhadri RK, Fast EM, Guo R, Schultz MJ, Vaisman N, Ortiz L, Bybee J, Slatko BE, Frydman HM. 2017. The Gut Commensal Microbiome of Drosophila melanogaster Is Modified by the Endosymbiont Wolbachia. mSphere 2.

60. Toni T, Welch D, Strelkowa N, Ipsen A, Stumpf MPH. 2009. Approximate Bayesian computation scheme for parameter inference and model selection in dynamical systems. J R Soc Interface 6:187-202.

61. Marjoram P. 2013. Approximation Bayesian Computation. OA Genet 1:853.

62. Gosset G. 2005. Improvement of Escherichia coli production strains by modification of the phosphoenolpyruvate:sugar phosphotransferase system. Microbial Cell Factories 4:14. method development. Biotechnol Adv 23:345-357.

64. Milo R, Phillips R. 2015. Cell Biology by the Numbers1 edition. Garland Science, New York, NY. 
bioRxiv preprint doi: https://doi org/10.1101/2021.01.07.425753; this version posted January 8, 2021. The copyright holder for this preprint (which was not certified by peer review) is the author/funder, who has granted bioRxiv a license to display the preprint in perpetuity. It is made available under aCC-BY-NC-ND 4.0 International license.

\section{Supplement}

\section{Supplemental Table S1: BioMe Materials}

\begin{tabular}{|c|c|c|c|}
\hline Component & Material & Vendor & Catnlogue \# \\
\hline Body Segment & $\begin{array}{l}\text { Machined: Serni-Clear White Polypropylene, 38' Thick } \\
\text { 3D-Printed: Dental SG Resin }\end{array}$ & $\begin{array}{c}\text { Machined: McMaster-Car } \\
\text { 3D-Printed FormLabs Dental SG Resin }\end{array}$ & $\begin{array}{c}\text { Machined: 8742K136 } \\
\text { 3D-Pinted: RSF2-DGOR-01 }\end{array}$ \\
\hline Transparent Base & Clear, Scratch-, UV, and Impact Resistant Polycarbonate, $1 f^{8}$ Thick & McMaster-Can & $8707 K 111$ \\
\hline Gasket & $\begin{array}{l}\text { Food-Grade High-Temperature Silicone Rubber Sheet, 1/32" Thick, } \\
\text { 40A Durometer (Medum Sol) }\end{array}$ & McMaster-Can & $5233 T 14$ \\
\hline ORings & High-Temperature Slicone ORing, $1 \mathrm{~mm}$ wide, $6 \mathrm{~mm}$ Inner Diameter & McMaster-Can & 95412A373 \\
\hline Threaded Rod & 18-8 Stainless Steel Threaded Rod, 6-32 Thread Size, 6' Long & McMaster-Can & 94758A102 \\
\hline Flange Nut & 18-8 Stainless Steel Flange Nut, 6-32 Thread Size & McMaster-Can & $86045 K 76$ \\
\hline Hat Head Screws & $\begin{array}{l}316 \text { Stainless Steel Hex Drive Hat Head Screw, } 82^{\circ} \text { Countersink, 4-40 } \\
\text { Thread Size, } 1 / 2^{n} \text { Long }\end{array}$ & McMaster-Can & 90585A204 \\
\hline Porous Membranes & 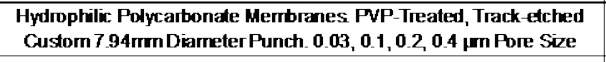 & Sterlitech & Custom Order \\
\hline Bottom Tray & Lid for 96384 mell Pkate, Clear, Polystyrene & Fisherbrand & $12-566-511$ \\
\hline Top Lid & $\begin{array}{l}96 \text { Well Cell Cullure Plate Hat Bottom - Lid, Non Pyrogenic, } \\
\text { Polystyrene }\end{array}$ & Costar & $07-200-720 A$ \\
\hline
\end{tabular}




\section{Supplemental Figure S2.1: Bromocresol Purple Diffusion Across the BioMe Plate}

765 Small-molecule dye (bromocresol purple) was placed on one side of the membrane in the BioMe plate for membranes of different pore sizes. Three replicates are shown here (overlapping) for each pore size. The
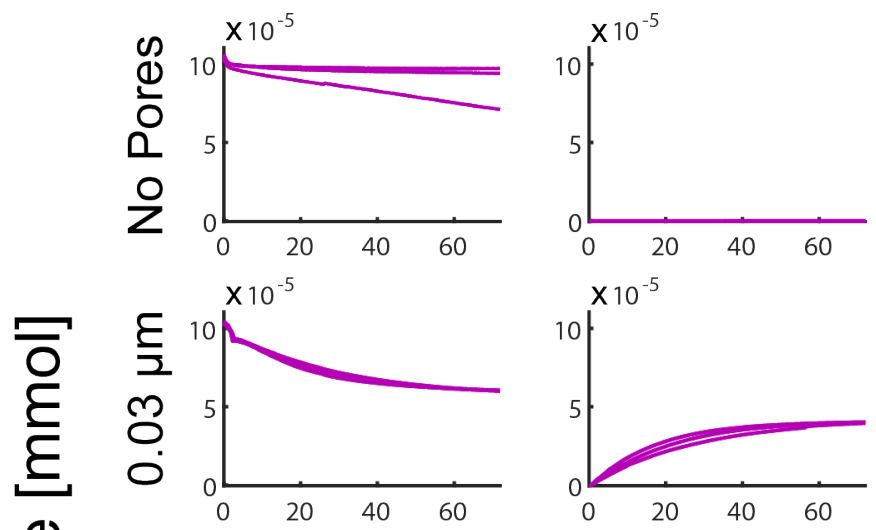

$\frac{1}{2}$
$\frac{2}{5}$
$\frac{0}{0}$
d
$\frac{0}{0}$
$\frac{0}{0}$
$\frac{0}{0}$
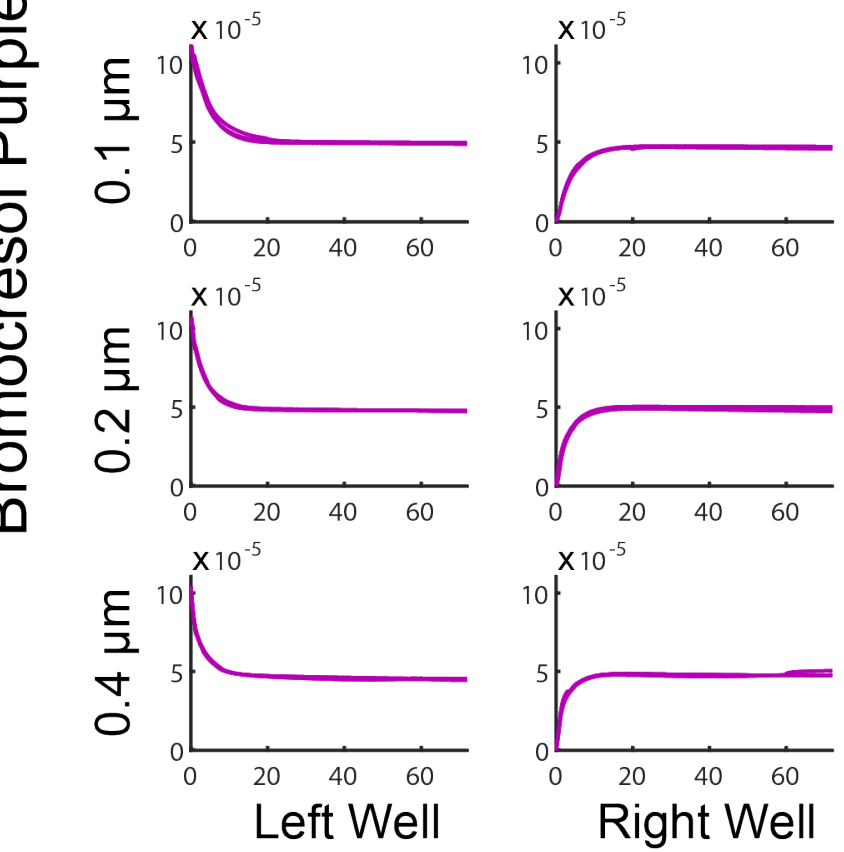

\section{Time [hours]} amount of dye was measured by optical density at the isosbestic point (optical density was converted to amount by a linear calibration shown in supplemental Figure S2.2). 
A

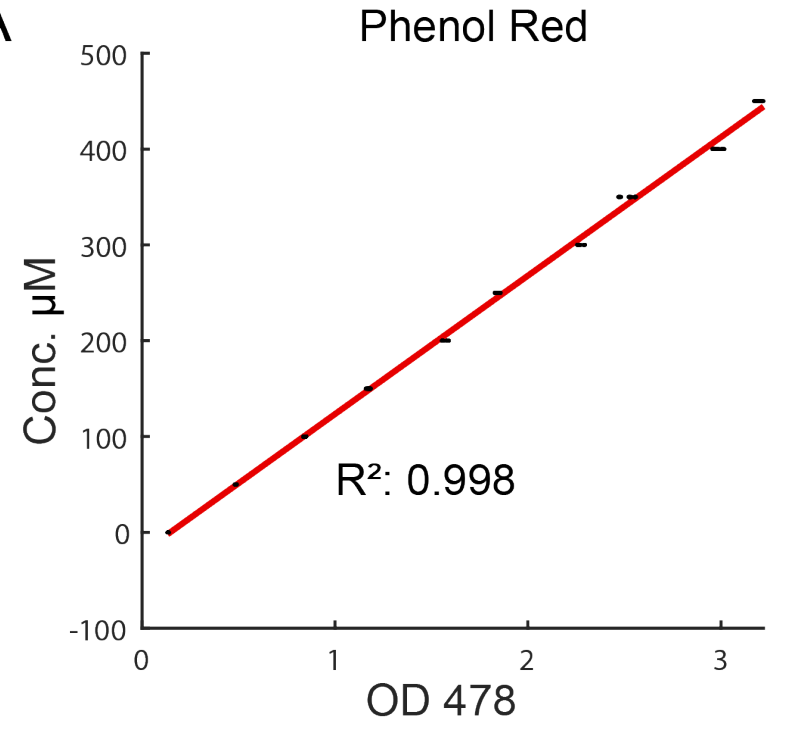

B

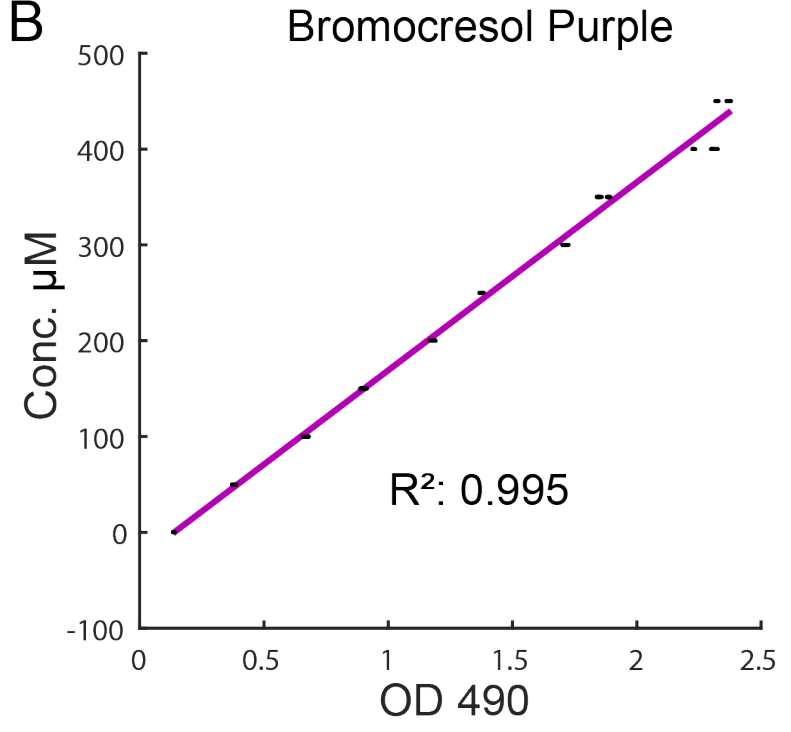

Supplemental Figure S2.2: Small Molecule Dye Calibration Curves

771 A linear calibration converting phenol red (A) and bromocresol purple (B) optical density at the isosbestic point to concentration. 
A

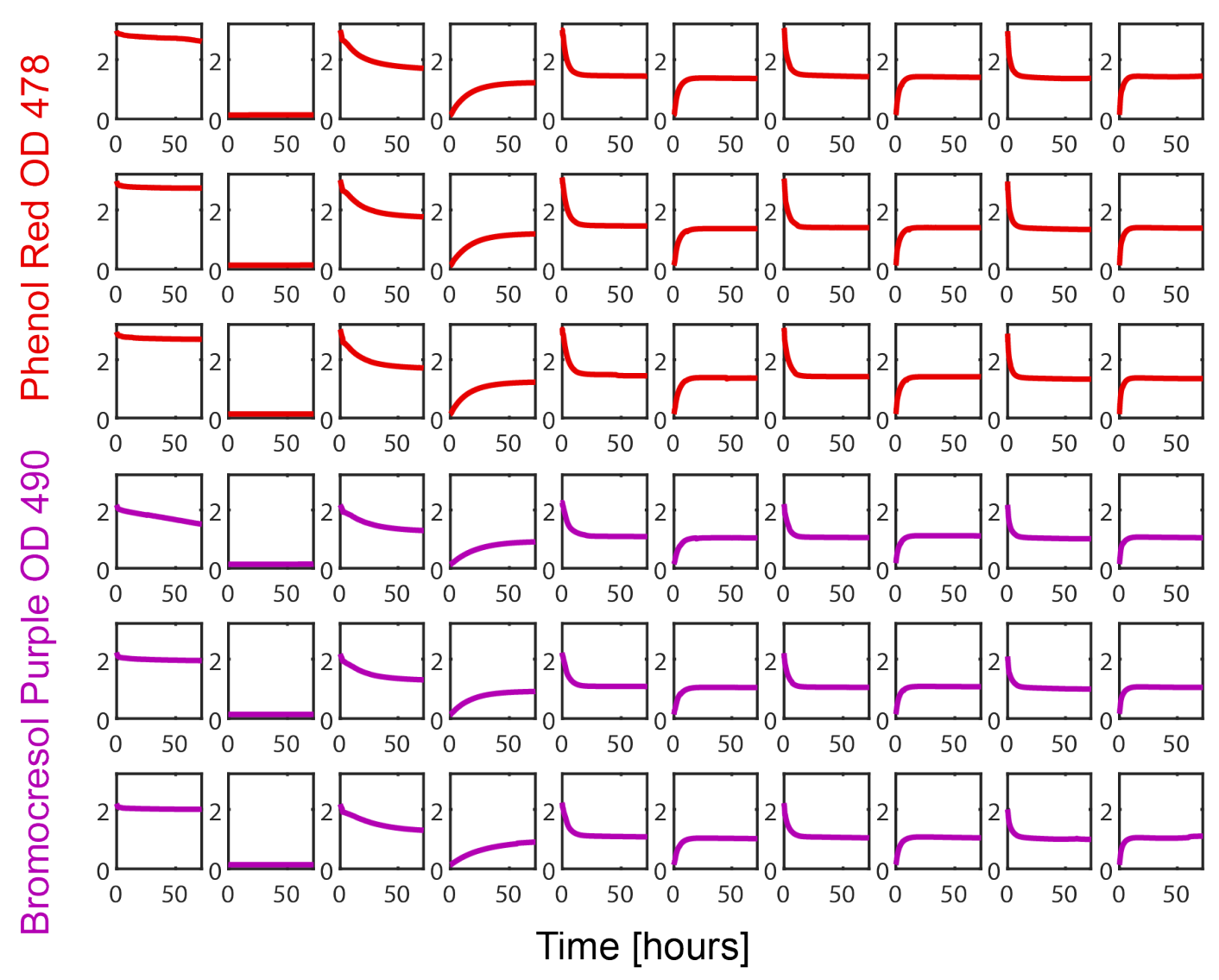

$\mathrm{B}$

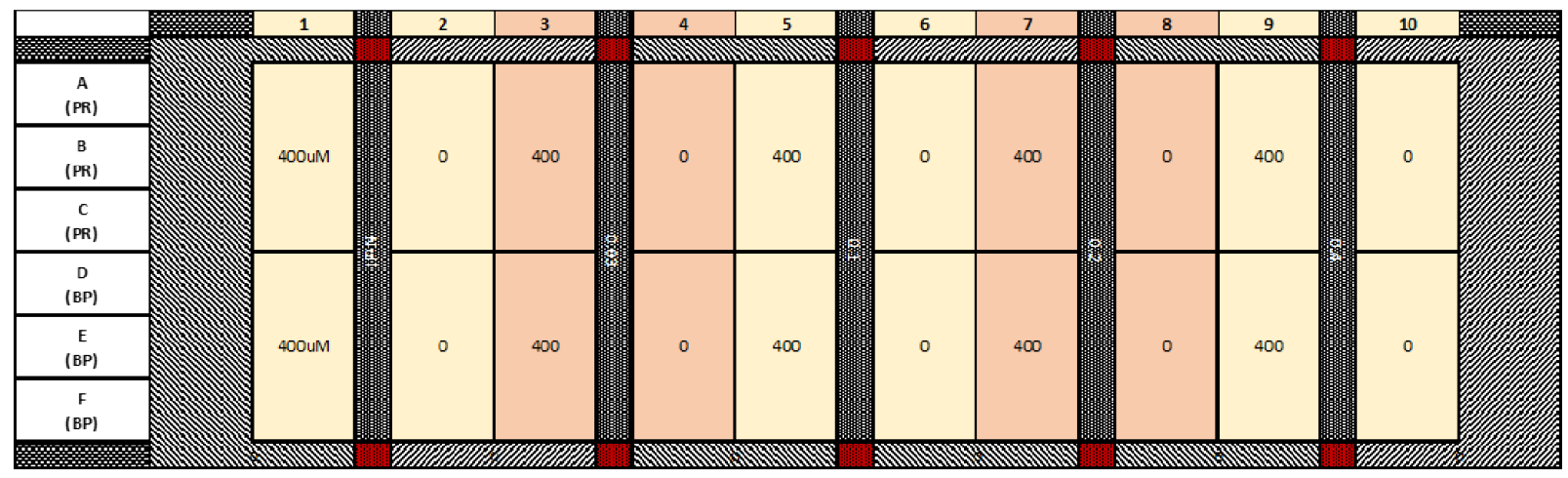

(A) The raw optical density readings from the diffusion experiment are shown. Red - phenol red, Purple bromocresol purple. (B) The layout of the experiment on the BioMe plate is shown. 
A

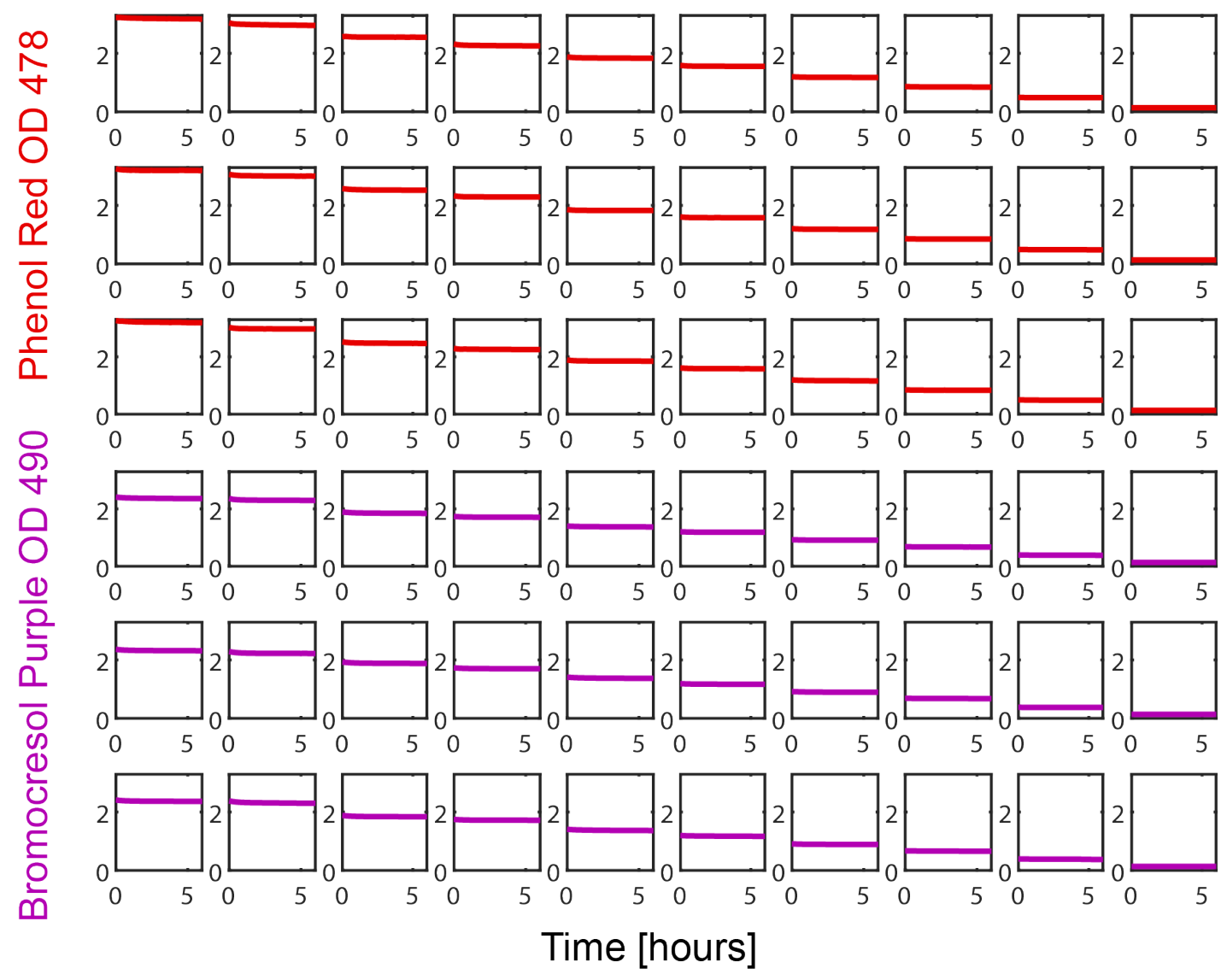

B

\begin{tabular}{|c|c|c|c|c|c|c|c|c|c|c|}
\hline & 1 & 2 & 3 & 4 & 5 & 6 & 7 & 8 & 9 & 10 \\
\hline $\begin{array}{c}\mathrm{A} \\
(\mathrm{PR})\end{array}$ & \multirow{3}{*}{ 450um } & \multirow{3}{*}{400} & \multirow{3}{*}{350} & \multirow{3}{*}{300} & \multirow{3}{*}{250} & \multirow{3}{*}{200} & \multirow{3}{*}{150} & \multirow{3}{*}{100} & \multirow{3}{*}{50} & \multirow{3}{*}{0} \\
\hline $\begin{array}{c}\mathrm{B} \\
\text { (PK) }\end{array}$ & & & & & & & & & & \\
\hline $\begin{array}{c}\mathrm{C} \\
\text { (PR) }\end{array}$ & & & & & & & & & & \\
\hline $\begin{array}{c}\mathrm{D} \\
\text { (BP) }\end{array}$ & \multirow{3}{*}{$450 \mathrm{um}$} & \multirow{3}{*}{400} & \multirow{3}{*}{350} & \multirow{3}{*}{300} & \multirow{3}{*}{250} & \multirow{3}{*}{200} & \multirow{3}{*}{150} & \multirow{3}{*}{100} & \multirow{3}{*}{50} & \multirow{3}{*}{0} \\
\hline$\underset{(B P)}{E}$ & & & & & & & & & & \\
\hline$\underset{(B P)}{F}$ & & & & & & & & & & \\
\hline
\end{tabular}

779 (A) The raw optical density readings from the dye calibration are shown. Red - phenol red, Purple -

780 bromocresol purple. (B) The layout of the experiment on the BioMe plate is shown. 
bioRxiv preprint doi: https://doi.org/10.1101/2021.01.07.425753; this version posted January 8, 2021. The copyright holder for this preprint

(which was not certified by peer review) is the author/funder, who has granted bioRxiv a license to display the preprint in perpetuity. It is made available under aCC-BY-NC-ND 4.0 International license.

\section{Supplemental Equations S1: Metabolite diffusion dynamics}

$782 A_{L\{R\}}(t)-\frac{K}{2}=\{-\} \frac{K}{2} e^{-2 \frac{d}{v} t}$

$783 A_{L\{R\}}(t)$ : the amount of metabolite in the left \{right $\}$ well at time $t[\mathrm{mmol}]$

$784 \quad K$ : the initial amount of metabolite [ $\mathrm{mmol}]$

785 d:diffusion constant $[L / h r]$

786 v:volume $[L]$ 


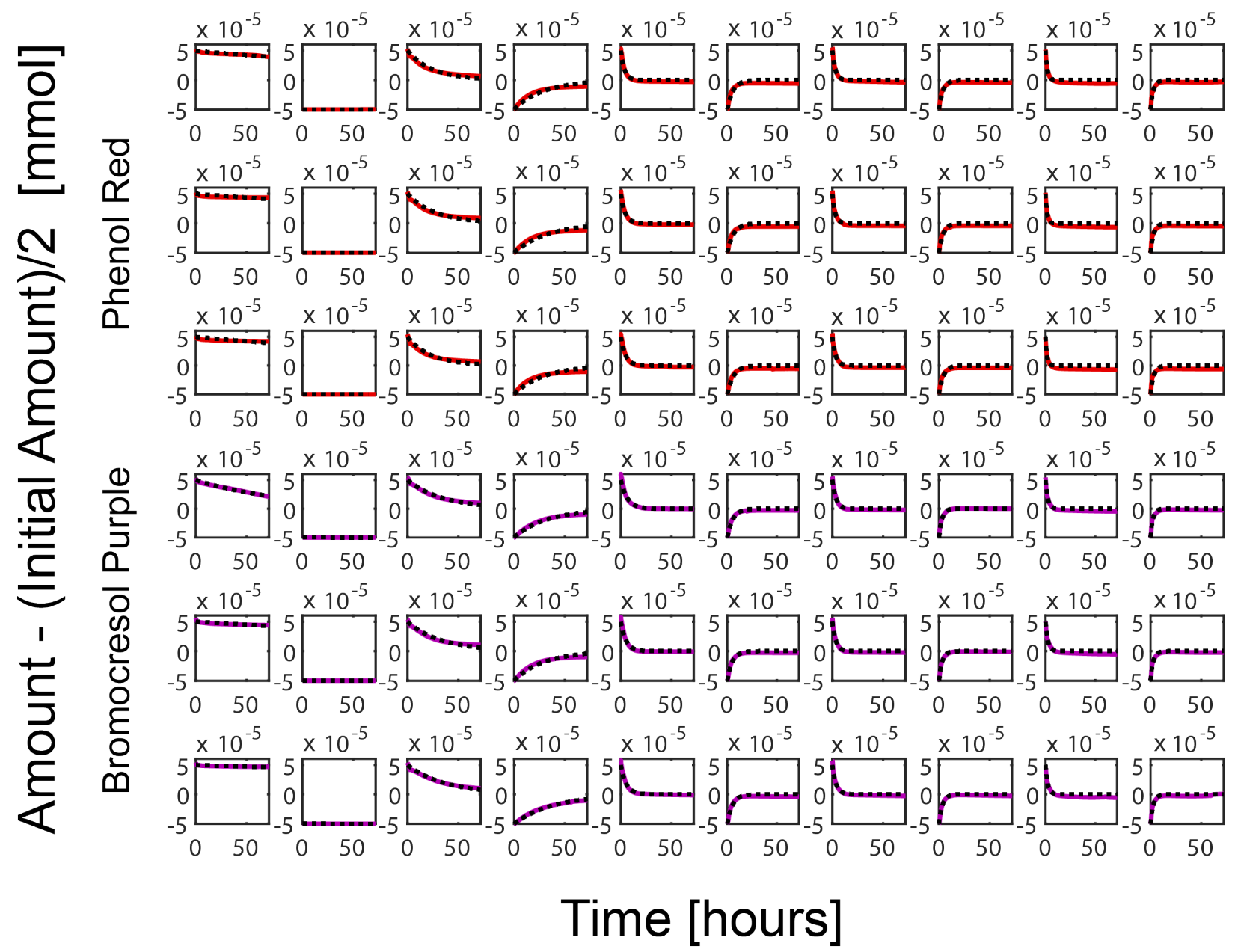

\section{Supplemental Figure S2.5: Metabolite diffusion curves fit to exponential function}

The experimental data from the chemical dye diffusion experiment was used to plot the value on the left hand side of supplemental Equations S1 (red - phenol red, purple - bromocresol purple). An exponential function was then fit to this data using the form of the right hand side of supplemental Equations S1

792 (dotted black line). 
A
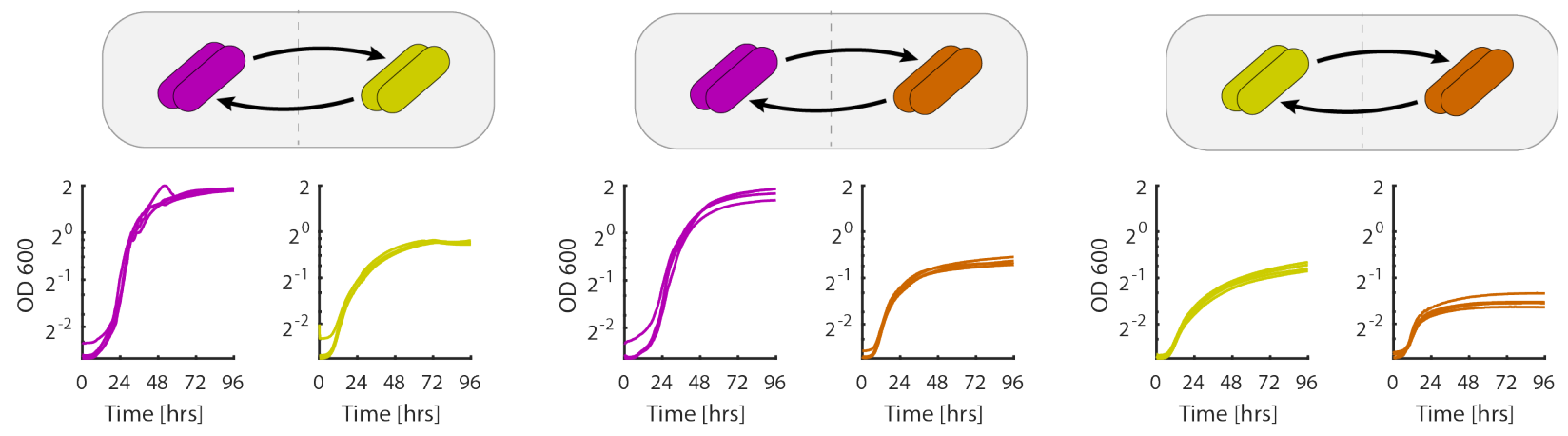

B
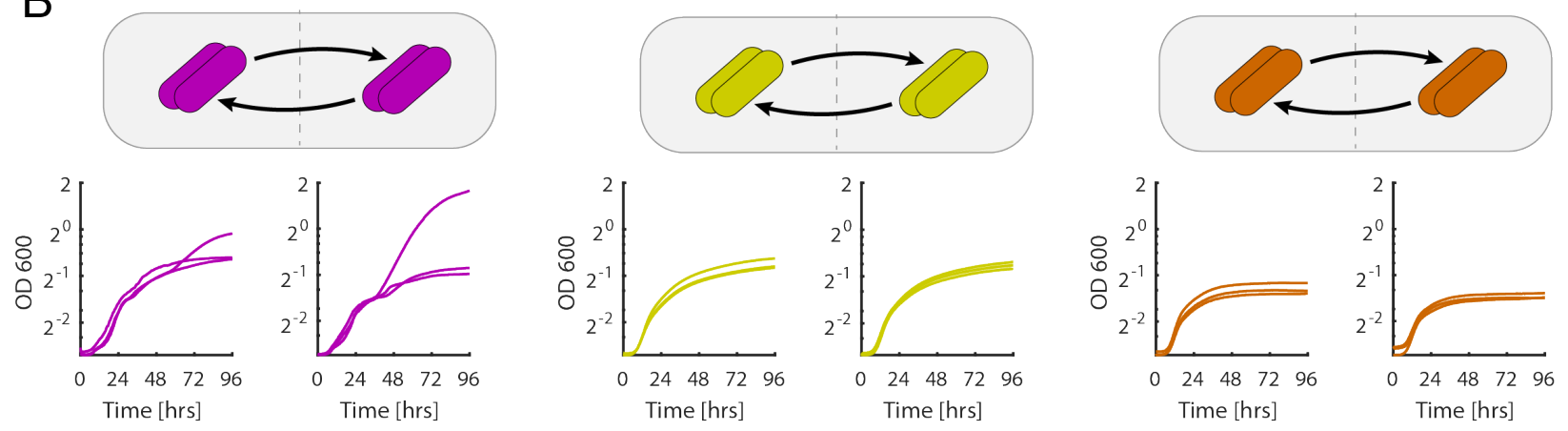

C
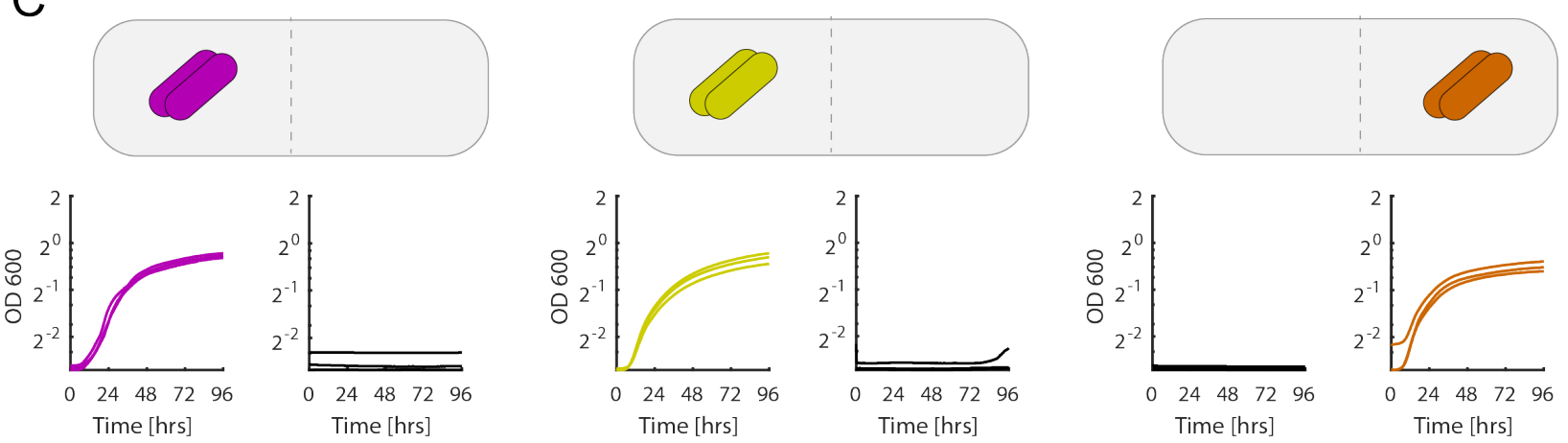

Supplemental Figure S3.1: Drosophila gut microbe interactions - all data and replicates

795 Growth curves and replicates for all co-culture experiments between A. oryzifermentans (purple), $L$. plantarum (yellow), and L. brevis (orange) are shown. The schematic demonstrates the type of (media-control). 
A
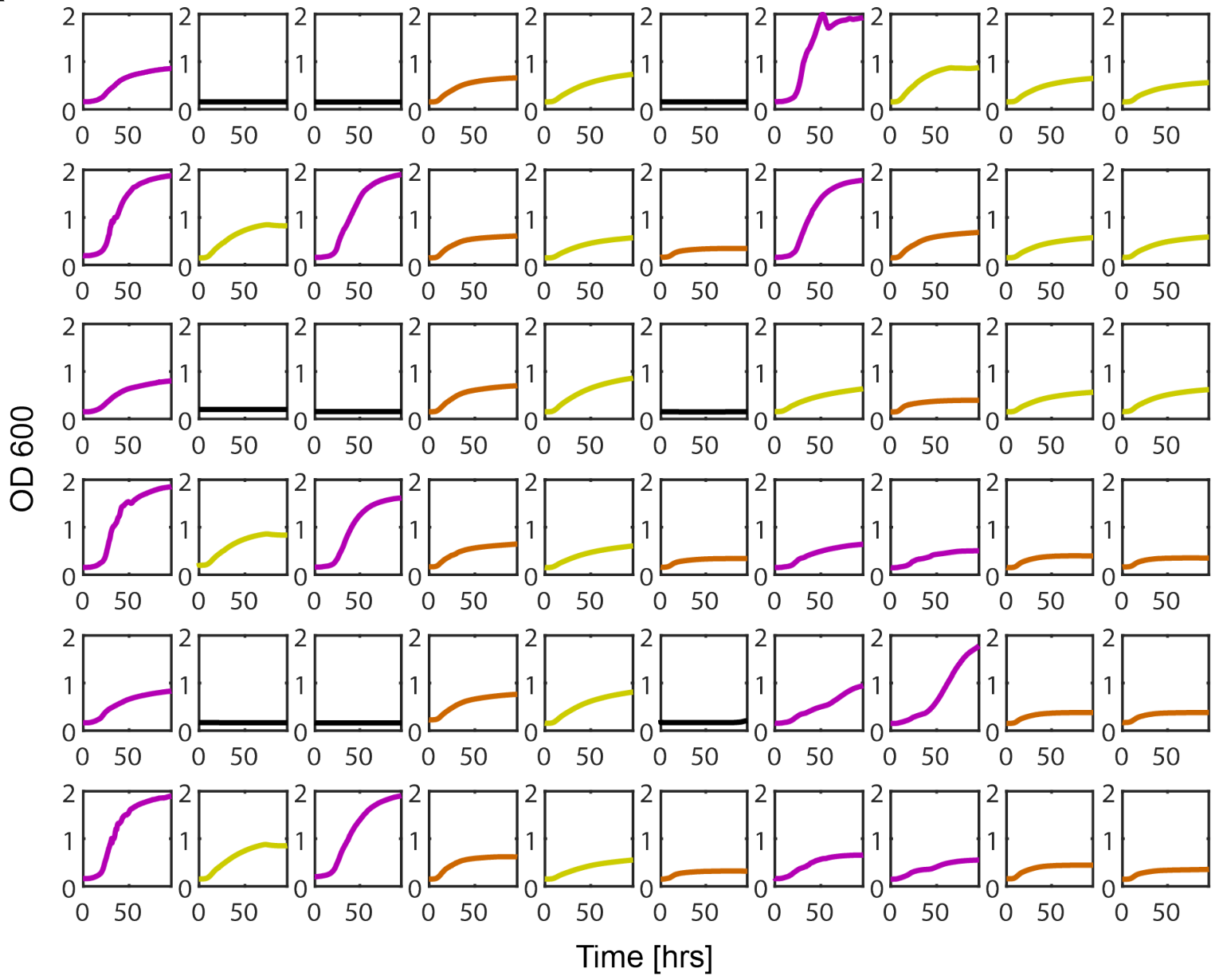

B

\begin{tabular}{|c|c|c|c|c|c|c|c|c|c|c|c|c|c|c|c|}
\hline & 1 & 5 & 2 & 3 & 1 & 4 & 5 & 1 & 6 & 7 & 15 & 8 & 9 & 15 & 10 \\
\hline A & $\mathrm{AO} 1$ & \multirow{6}{*}{$\mid \begin{array}{l}0 \\
\frac{\mathrm{m}}{5} \\
3\end{array}$} & & & \multirow{6}{*}{ 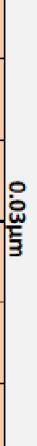 } & LB1 & LP1 & \multirow{6}{*}{ 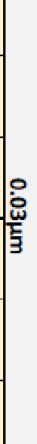 } & & $\mathrm{AO} 4$ & \multirow{6}{*}{$\begin{array}{l}\stackrel{0}{0} \\
\dot{\omega} \\
\frac{1}{3}\end{array}$} & LP4 & LP1 & \multirow{6}{*}{ 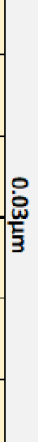 } & LP1 \\
\hline B & $\mathrm{AO} 1$ & & LP1 & $\mathrm{AO} 1$ & & LB1 & LP1 & & LB 1 & $\mathrm{AO} 4$ & & LB4 & LP2 & & LP2 \\
\hline C & $\mathrm{AO} 2$ & & & & & LB2 & LP2 & & & LP4 & & LB4 & LP3 & & LP3 \\
\hline D & $\mathrm{AO} 2$ & & LP2 & $\mathrm{AO} 2$ & & LB2 & LP2 & & LB2 & $\mathrm{AO} 1$ & & $\mathrm{AO} 1$ & LB1 & & LB1 \\
\hline $\mathbf{E}$ & $\mathrm{AO3}$ & & & & & LB3 & LP3 & & & $\mathrm{AO} 2$ & & $\mathrm{AO} 2$ & LB2 & & LB2 \\
\hline $\mathbf{F}$ & $\mathrm{AO} 3$ & & LP3 & $\mathrm{AO3}$ & & LB3 & LP3 & & LB3 & $\mathrm{AO} 3$ & & $\mathrm{AO} 3$ & LB3 & & LB3 \\
\hline
\end{tabular}

Supplemental Figure S3.2: Drosophila gut microbe interactions - plate layout

801

802

803

804

805
(A) The raw experimental optical density measurements for all cultures are shown for co-culture experiments of A. oryzifermentans (purple), L. plantarum (yellow), and L. brevis (orange). (B) The layout of the experiment on the BioMe plate is shown. Two outliers for the $A$. oryzifermentans v.s. self-cocultures were left out of the analysis done in figure 3, these are the curves from the interacting wells E7 and E8. 
A
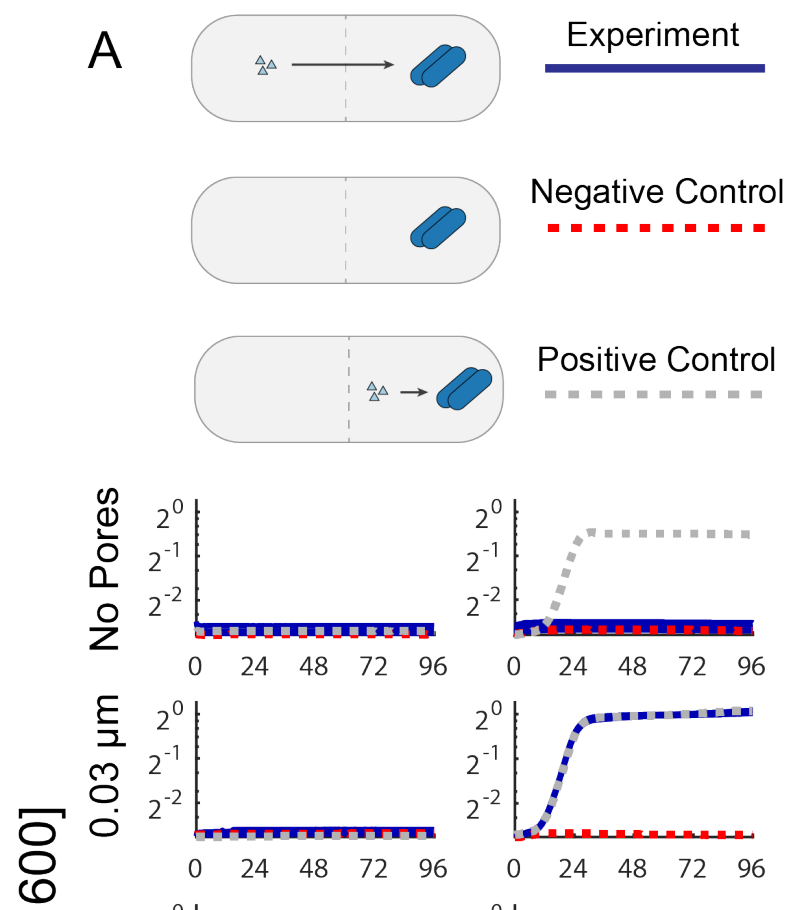

$\frac{0}{3}$
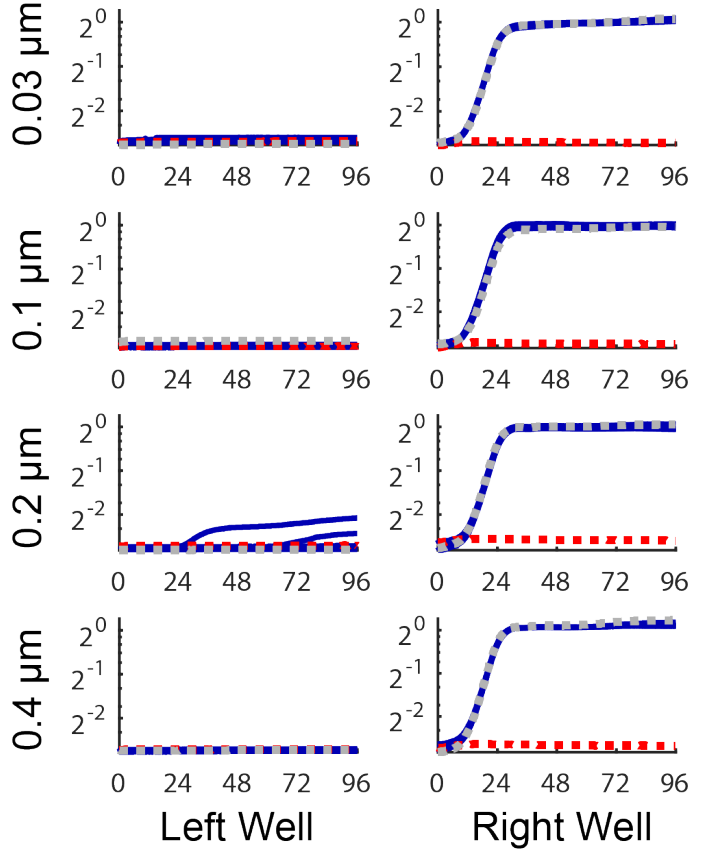

\section{Time [hours]}

B
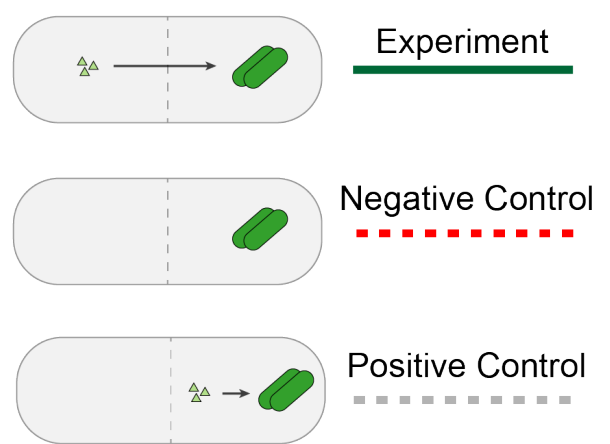

Positive Control

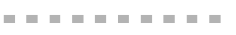
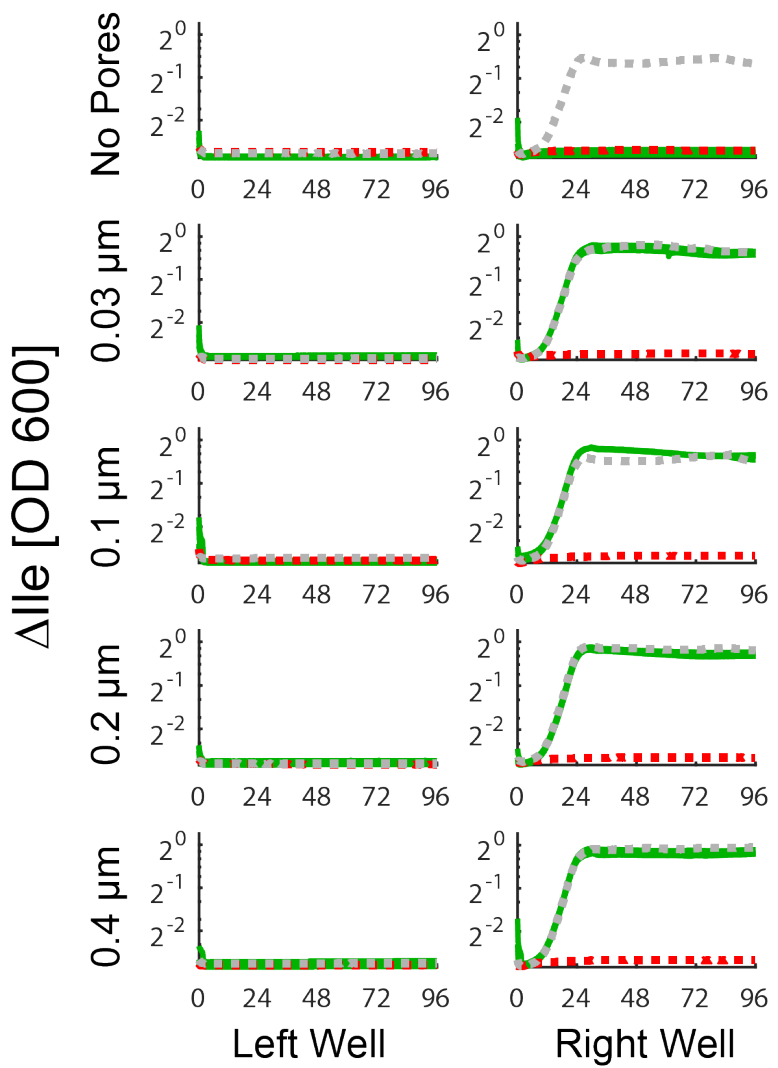

Time [hours]

807 Supplemental Figure S4.1: $\boldsymbol{E}$. coli auxotroph amino acid diffusion experiments - all data and

\section{8 replicates}

809 The growth of $\Delta$ Lys (A) and $\Delta$ Ile (B) are shown for five different pore sizes (No Pores, 0.03, 0.1, 0.2, and $0.4 \mu \mathrm{m}$ ) alongside positive and negative control growth curves. Blue $-\Delta$ Lys, Green $-\Delta \mathrm{Ile}$, Red - 


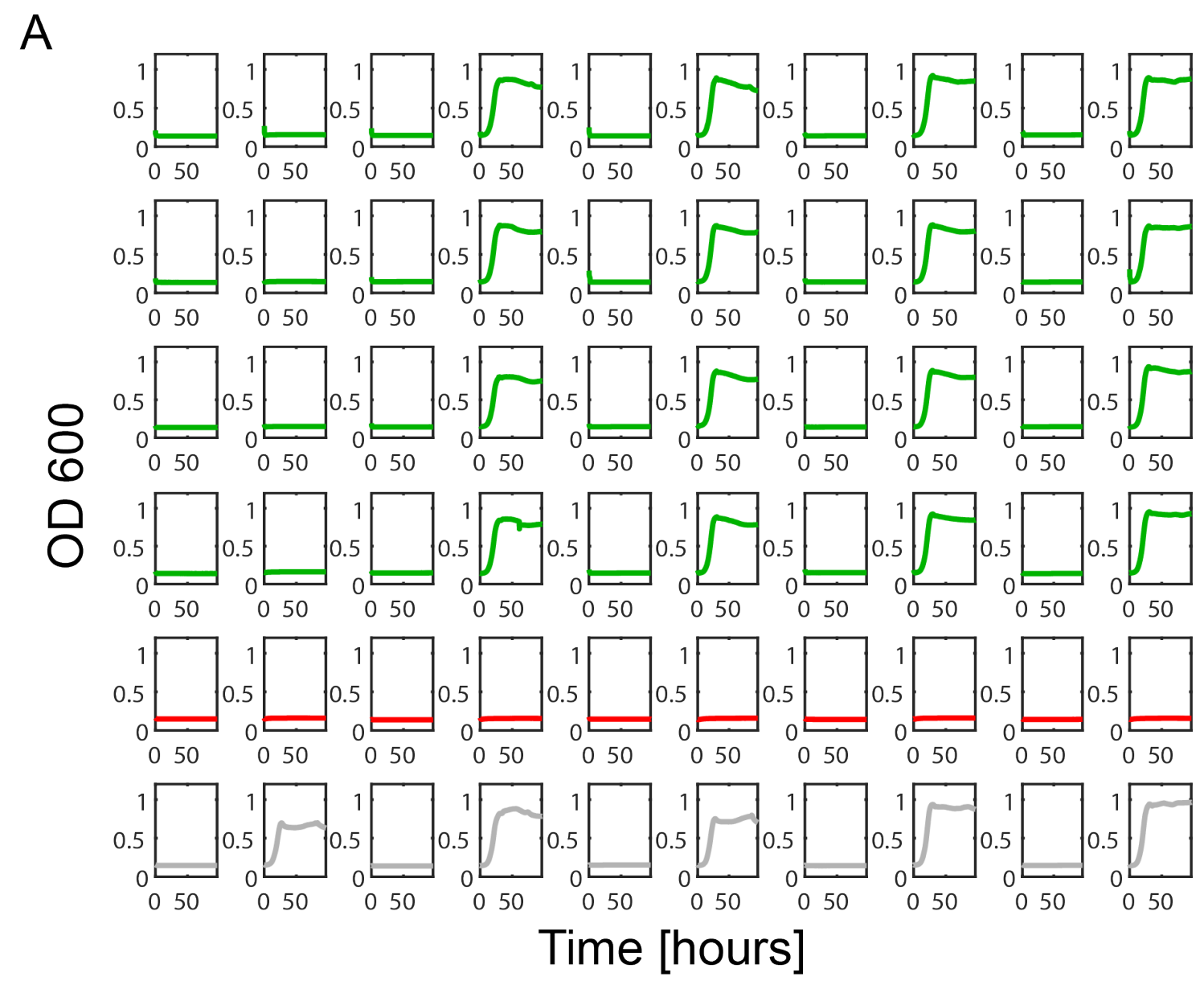

B

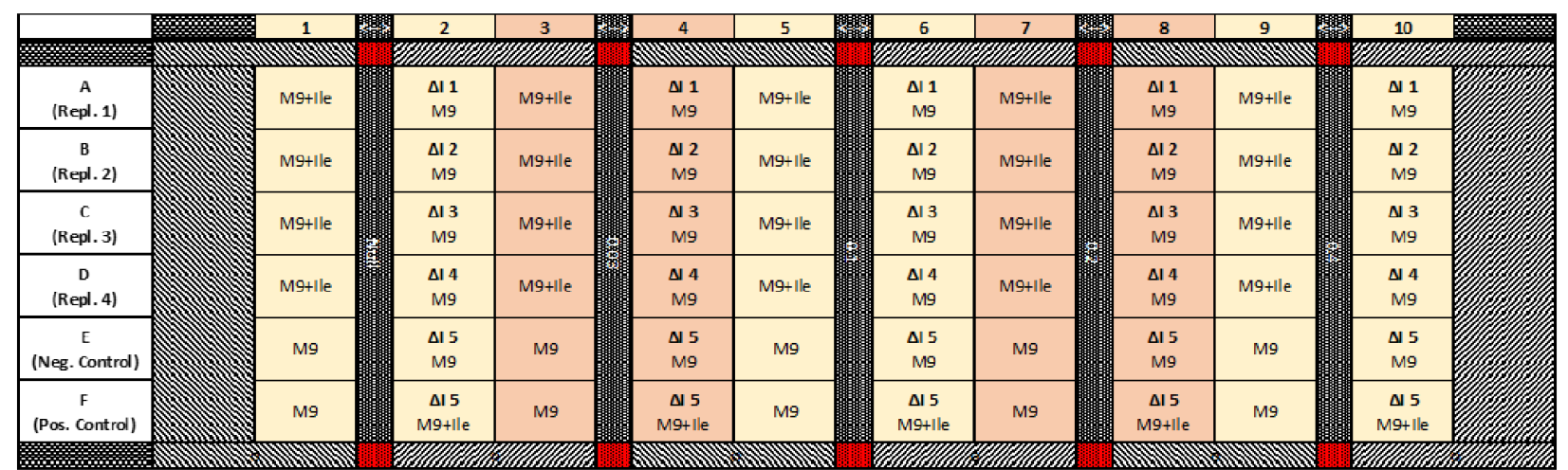

814 (A) The raw optical density readings for the E. coli $\Delta$ Ile auxotroph isoleucine diffusion experiment are 815 shown. Green - $\Delta$ Ile, Red - negative controls, Gray - positive controls. (B) The layout of the experiment 816 on the BioMe plate is shown. 

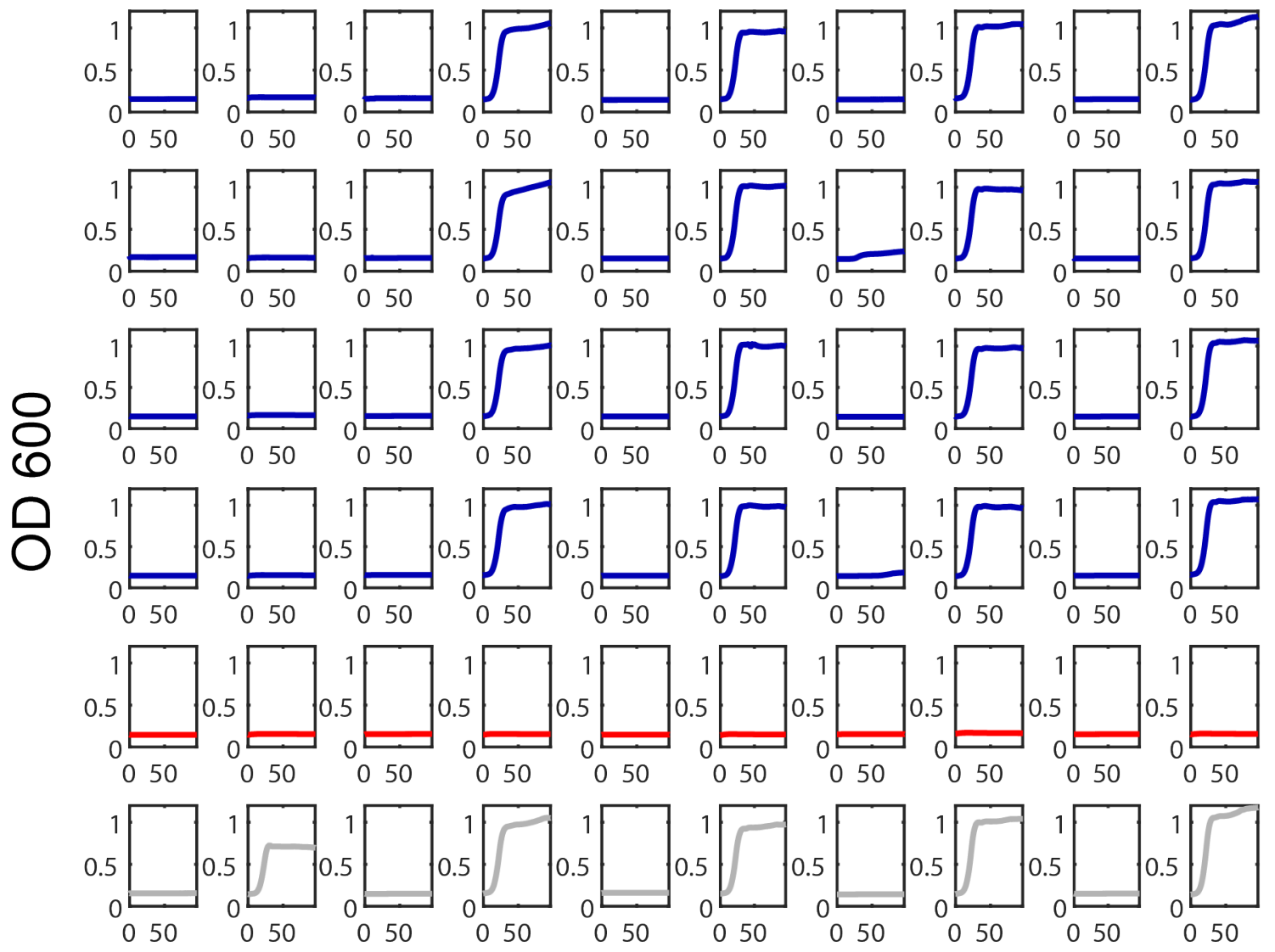

Time [hours]

818 Supplemental Figure S4.3: E. coli $\Delta$ Lys auxotroph amino acid diffusion experiments - plate layout

819 (A) The raw optical density readings for the E. coli $\Delta$ Lys auxotroph lysine diffusion experiment are shown. The layout of the experiment on the BioMe plate is the same as that shown for the $\Delta$ Ile experiment, with $\Delta$ Lys replacing $\Delta$ Ile and lysine replacing isoleucine. Blue $-\Delta$ Lys, Red - negative controls, Gray - positive controls. 


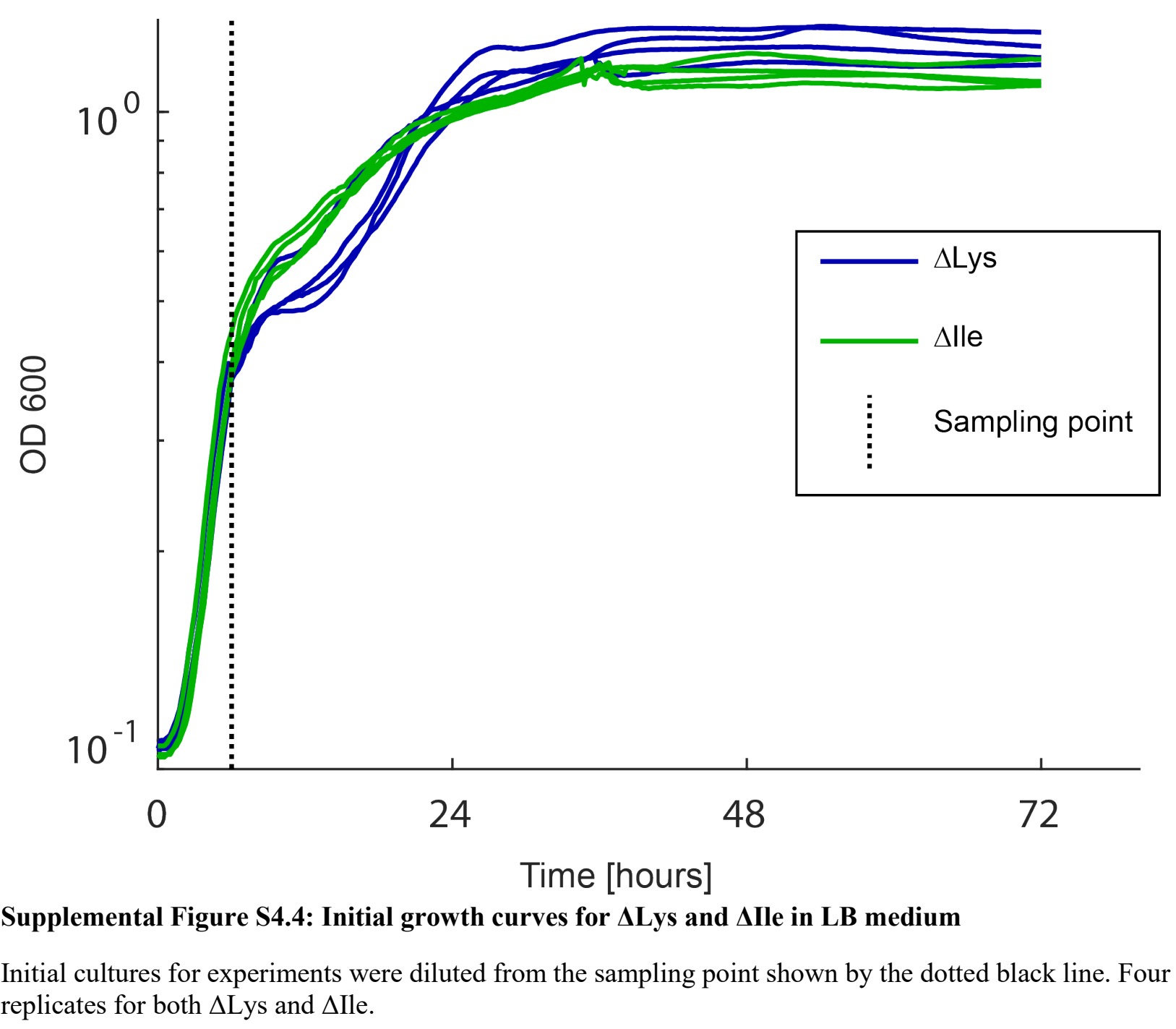

825 Initial cultures for experiments were diluted from the sampling point shown by the dotted black line. Four 826 replicates for both $\Delta$ Lys and $\Delta$ Ile. 

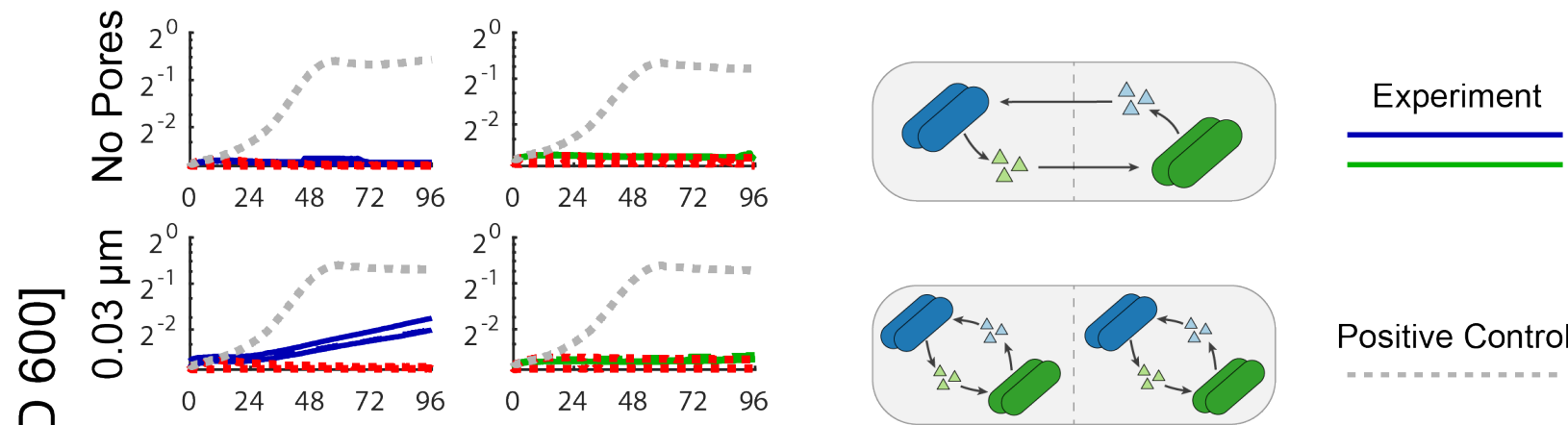

Positive Control
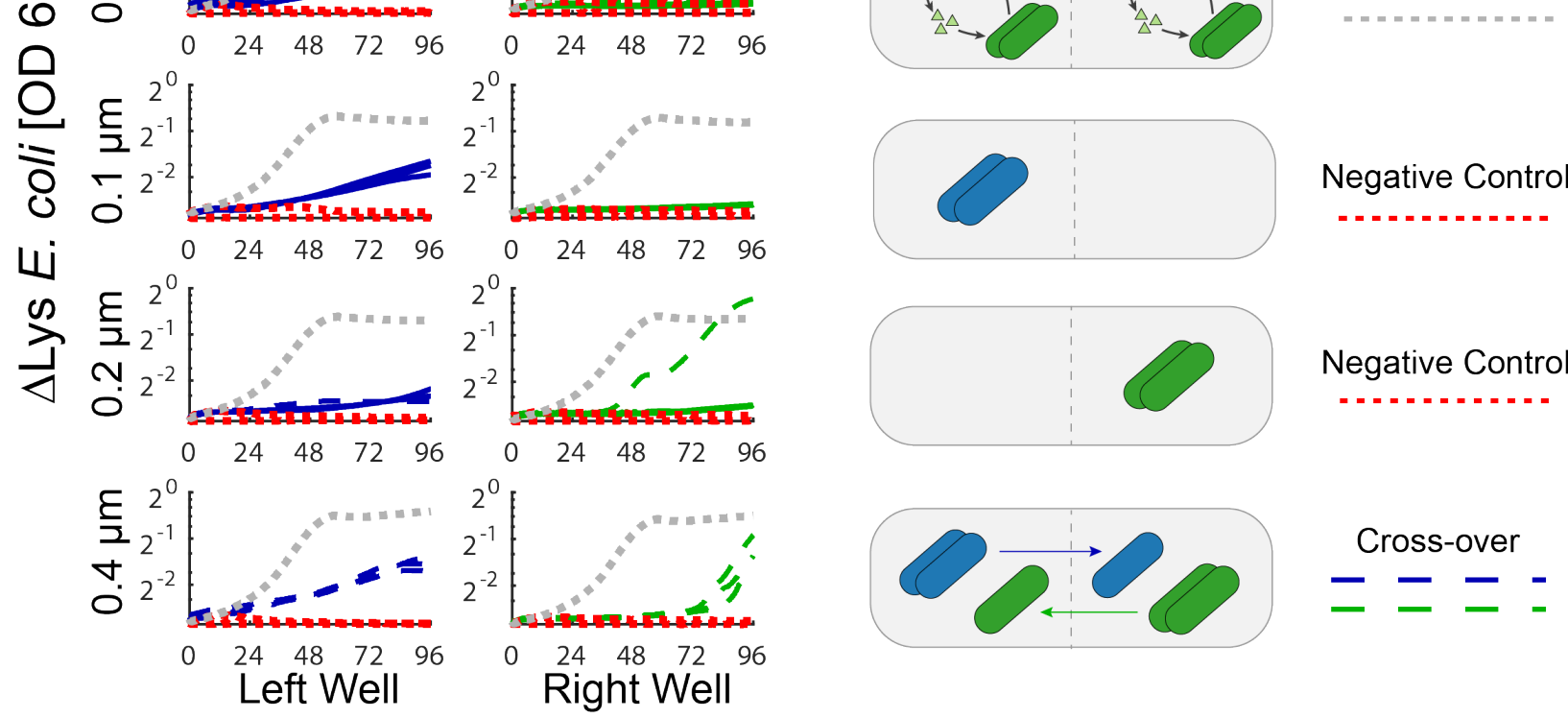

Negative Control

Negative Control
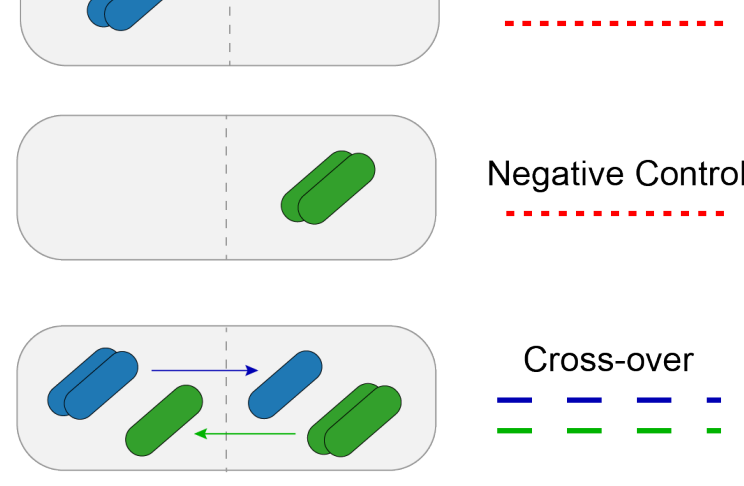

Cross-over

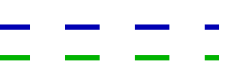

\section{Time [hours]}

829 The growth curves of $\Delta$ Lys and $\Delta$ Ile co-cultures are shown for 5 different pore sizes (No Pores, $0.03,0.1$, $830 \quad 0.2$, and $0.4 \mu \mathrm{m}$ ) alongside positive and negative controls. Dotted lines indicate cross-over of auxotrophs 831 as indicated in supplemental Table S3. Blue $-\Delta$ Lys, Green $-\Delta$ Ile, Red - negative controls, Gray 832 positive controls. 


\section{A}
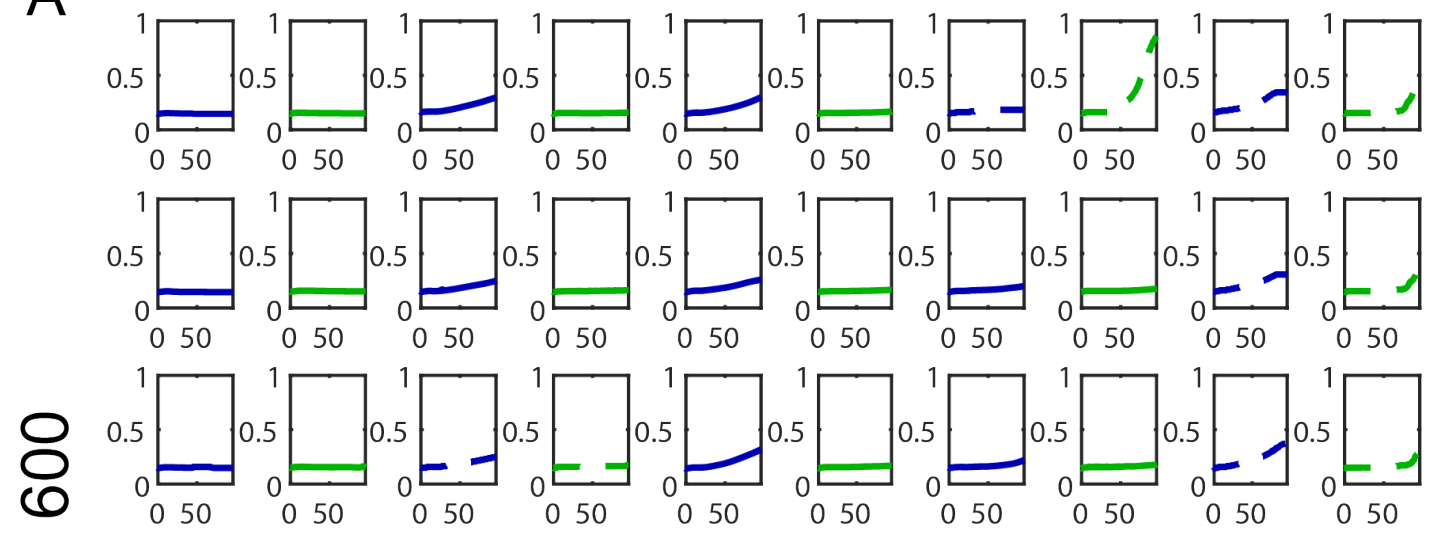

○
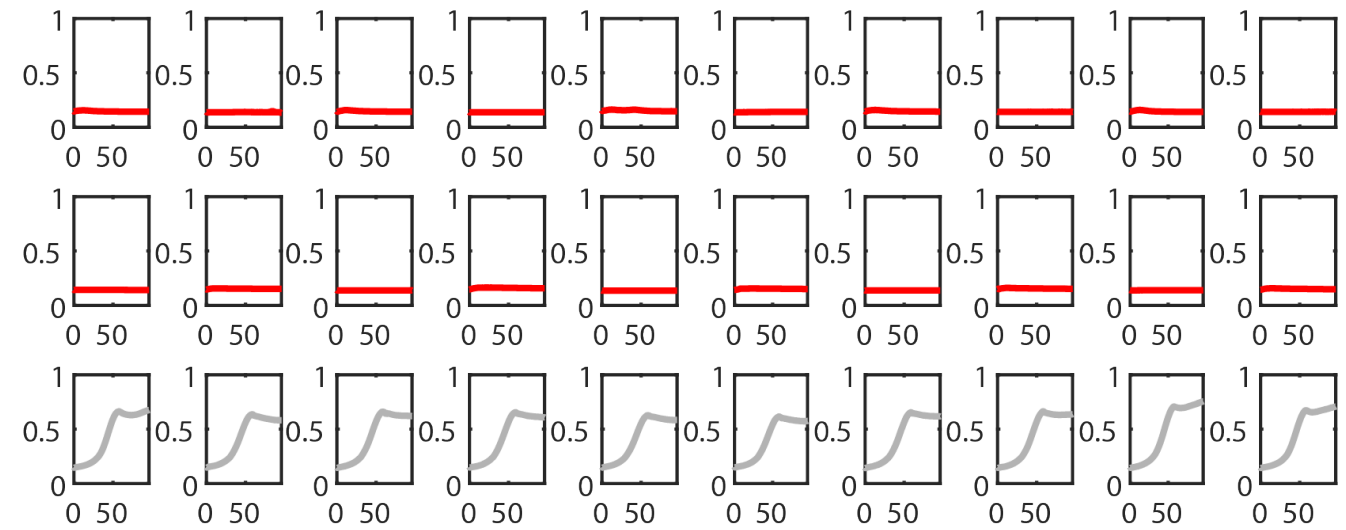

\section{Time [hours]}

\begin{tabular}{|c|c|c|c|c|c|c|c|c|c|c|}
\hline & 1 & 2 & 3 & 4 & 5 & 6 & 7 & 8 & 9 & 10 \\
\hline & & & & & & & & & & \\
\hline $\begin{array}{c}\text { A } \\
\text { (Repl. 1) }\end{array}$ & $\begin{array}{l}\Delta K 1 \\
\text { M9 }\end{array}$ & $\begin{array}{l}\Delta 1 \\
\text { M } 1\end{array}$ & $\begin{array}{l}\Delta K 1 \\
\text { M9 }\end{array}$ & $\begin{array}{l}\Delta \mathbf{1} 1 \\
\text { M9 }\end{array}$ & $\begin{array}{c}\Delta K 1 \\
\text { M9 }\end{array}$ & $\begin{array}{l}\Delta 1 \\
\text { M9 }\end{array}$ & $\begin{array}{c}\Delta K_{1} 1 \\
\text { M9 }\end{array}$ & $\begin{array}{l}\Delta \mathbf{1} 1 \\
\text { M9 }\end{array}$ & $\begin{array}{c}\Delta K_{1} 1 \\
M 9\end{array}$ & $\begin{array}{l}\Delta 11 \\
\text { M9 }\end{array}$ \\
\hline $\begin{array}{c}\text { B } \\
\text { (Repl. 2) }\end{array}$ & $\begin{array}{c}\Delta K 2 \\
\text { M9 }\end{array}$ & $\begin{array}{l}\Delta 2 \\
\text { M } 9\end{array}$ & $\begin{array}{l}\Delta K 2 \\
\text { M9 }\end{array}$ & $\begin{array}{l}\Delta 2 \\
\text { M9 }\end{array}$ & $\begin{array}{c}\Delta K 2 \\
M 9\end{array}$ & $\begin{array}{l}\Delta 2 \\
\text { M9 }\end{array}$ & $\begin{array}{l}\Delta K 2 \\
M 9\end{array}$ & $\begin{array}{l}\Delta 12 \\
\text { M9 }\end{array}$ & $\begin{array}{c}\Delta K 2 \\
\text { M9 }\end{array}$ & $\begin{array}{l}\Delta 12 \\
\text { M9 }\end{array}$ \\
\hline $\begin{array}{c}c \\
0\end{array}$ & $\Delta K_{3}$ & $\Delta 3$ & $\Delta K_{3} 3$ & $\Delta \mathbf{3}$ & $\Delta K_{3}$ & $\Delta 3$ & $\Delta K_{3}$ & $\Delta 3$ & $\Delta K_{3}$ & $\Delta 3$ \\
\hline 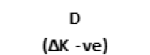 & $\begin{array}{c}\Delta \mathrm{K} 4 \\
\mathrm{Mg}\end{array}$ & M9 & $\begin{array}{l}\Delta K 4 \\
\text { M9 }\end{array}$ & M9 & $\begin{array}{c}\Delta K 4 \\
\text { M9 }\end{array}$ & M9 & $\begin{array}{c}\Delta K 4 \\
\text { M9 }\end{array}$ & M9 & $\begin{array}{c}\Delta K 4 \\
\text { M9 }\end{array}$ & M9 \\
\hline $\begin{array}{c}E \\
(\Delta I-v e)\end{array}$ & M9 & $\begin{array}{l}\Delta 4 \\
\text { M9 }\end{array}$ & M9 & $\begin{array}{l}\mathrm{Nl} 4 \\
\mathrm{M} 9\end{array}$ & M9 & $\begin{array}{l}\text { N } 4 \\
\text { M9 }\end{array}$ & M9 & $\begin{array}{l}\Delta / 4 \\
\text { M9 }\end{array}$ & M9 & $\begin{array}{l}\Delta 14 \\
\text { M9 }\end{array}$ \\
\hline $\begin{array}{c}\text { F } \\
\text { (Same Well +ve) }\end{array}$ & $\begin{array}{c}\Delta K 4+\Delta / 4 \\
M 9\end{array}$ & $\begin{array}{c}\Delta \mathbf{K} 4+\Delta / 4 \\
\text { M9 }\end{array}$ & $\begin{array}{c}\Delta K 4+\Delta / 4 \\
M 9\end{array}$ & $\begin{array}{c}\Delta K 4+\Delta / 4 \\
\text { M } 9\end{array}$ & $\begin{array}{c}\Delta K 4+\Delta / 4 \\
\text { M } 9\end{array}$ & $\begin{array}{c}\Delta \mathrm{K} 4+\Delta / 4 \\
\mathrm{M} 9\end{array}$ & $\begin{array}{c}\Delta \mathbf{K} 4+\Delta / 4 \\
\mathrm{M} 9\end{array}$ & $\begin{array}{c}\Delta \mathrm{K} 4+\Delta / 4 \\
\mathrm{M} 9\end{array}$ & $\begin{array}{c}\Delta \mathrm{K} 4+\Delta / 4 \\
\mathrm{M} 9\end{array}$ & $\begin{array}{c}\Delta K 4+\Delta / 4 \\
\mathrm{M} 9\end{array}$ \\
\hline
\end{tabular}

835 (A) The raw optical density measurements for the E. coli auxotroph co-culture experiment are shown.

\section{Supplemental Figure S5.2: E. coli auxotroph co-culture experiments - plate layout}

Dotted lines indicate cross-over of auxotrophs as indicated in supplemental Table S3. Blue $-\Delta$ Lys, Green $-\Delta$ Ile, Red - negative controls, Gray - positive controls. (B) The layout of the experiment on the BioMe plate is shown. 


\section{Supplemental Table S2: Cross-over assay}

840 A selective platting assay at the end of the E. coli auxotroph co-culture experiment was performed to

841 identify when organisms had crossed into the adjacent well of the BioMe plate. Black letters indicate that 842 the correct auxotroph was identified in that well, and red letters indicate that the incorrect auxotroph was 843 found in the well.

844

\begin{tabular}{|c|c|c|c|c|c|c|c|c|c|c|c|c|c|c|c|}
\hline & 1 & & 2 & 3 & & 4 & 5 & & 6 & 7 & & 8 & 9 & & 10 \\
\hline $\begin{array}{c}\text { A } \\
\text { (Repl. 1) }\end{array}$ & $\Delta K$ & \multirow{5}{*}{$\begin{array}{l}z \\
z \\
0 \\
0 \\
0 \\
b\end{array}$} & $\Delta l$ & $\Delta \mathrm{K}$ & \multirow{6}{*}{ 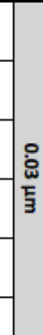 } & $\Delta l$ & $\Delta K$ & \multirow{6}{*}{ 苞 } & $\Delta l$ & $\Delta \mathrm{K}$ & \multirow{6}{*}{ i } & $\Delta K \& \Delta I$ & $\Delta K$ & \multirow{6}{*}{ 章 } & $\Delta K \& \Delta I$ \\
\hline $\begin{array}{c}\text { B } \\
\text { (Repl. 2) }\end{array}$ & $\Delta K$ & & $\Delta l$ & $\Delta \mathrm{K}$ & & $\Delta l$ & $\Delta K$ & & $\Delta l$ & $\Delta K$ & & $\Delta l$ & $\Delta \mathrm{K}$ & & $\Delta K \& \Delta I$ \\
\hline $\begin{array}{c}\text { C } \\
\text { (Repl. 3) }\end{array}$ & $\Delta K$ & & $\Delta l$ & $\Delta K \& \Delta I$ & & $\Delta l$ & $\Delta K$ & & $\Delta l$ & $\Delta \mathrm{K}$ & & $\Delta l$ & $\Delta K$ & & $\Delta K \& \Delta I$ \\
\hline $\begin{array}{c}\text { D } \\
\text { (Repl. 4) }\end{array}$ & $\Delta K$ & & & $\Delta K$ & & & $\Delta K$ & & & $\Delta \mathrm{K}$ & & & $\Delta \mathrm{K}$ & & \\
\hline $\begin{array}{c}\text { E } \\
\text { (-ve Control) }\end{array}$ & & & $\Delta l$ & & & $\Delta l$ & & & $\Delta$ & & & $\Delta l$ & & & $\Delta l$ \\
\hline $\begin{array}{c}\mathbf{F} \\
\text { (+ve Control) }\end{array}$ & $\Delta K \& \Delta I$ & & $\Delta K \& \Delta I$ & $\Delta K \& \Delta I$ & & $\Delta K \& \Delta I$ & $\Delta K \& \Delta \mathrm{I}$ & & $\Delta K \& \Delta I$ & $\Delta K \& \Delta I$ & & $\Delta K \& \Delta \mathrm{I}$ & $\Delta K \& \Delta I$ & & $\Delta K \& \Delta I$ \\
\hline
\end{tabular}


$849 G_{L\{R\}}[\mathrm{mmol}]$ (glucose left $\{$ right $\}$ )

$850 K_{L\{R\}}[\mathrm{mmol}]($ Lysine left $\{$ right $\})$

$851 I_{L\{R\}}[\mathrm{mmol}]$ (Isoleucine left $\{$ right $\}$ )

$852 B_{L\{R\}}^{K}[\mathrm{~g}]($ Biomass of $E$. coli $\Delta$ Lysine left $\{$ right $\})$

$853 B_{L\{R\}}^{I}[\mathrm{~g}]$ (Biomass of $E$. coli $\Delta$ Isoleucine left $\{$ right $\}$ )

854

Parameters [units]:

Kinetics

$\operatorname{vmax}_{G}[\mathrm{mmol} /(\mathrm{hr} * \mathrm{~g})]$

$\operatorname{vmax}_{K}[\mathrm{mmol} /(\mathrm{hr} * \mathrm{~g})]$

$\max _{I}\left[\mathrm{mmol} /\left(\mathrm{hr}^{*} \mathrm{~g}\right)\right]$

$860 k m_{G}[\mathrm{mmol} / \mathrm{L}]$

$861 \quad k m_{K}[\mathrm{mmol} / \mathrm{L}]$

$862 k m_{I}[\mathrm{mmol} / \mathrm{L}]$

863

864 Biomass Stoichiometry

$865 z_{G}[\mathrm{~g} / \mathrm{mmol}]$

$866 \quad z_{K}[\mathrm{~g} / \mathrm{mmol}]$

$867 \quad z_{I}[\mathrm{~g} / \mathrm{mmol}]$

868

869

870

871

872

873

874

875

876

877

878

879

Leakage Stoichiometry

$y_{K}[\mathrm{mmol} / \mathrm{g}]$

$y_{I}[\mathrm{mmol} / \mathrm{g}]$

880 - Growth

Volume

$v$ [L]

880 Growth

$881 \quad V^{B_{L\{R\}}^{K}}=\min \left(\frac{\operatorname{vmax}_{G} * \frac{G_{L\{R\}}}{v}}{k m_{G}+\frac{G_{L\{R\}}}{v}} * z_{G}, \frac{\operatorname{vmax}_{K} * \frac{K_{L\{R\}}}{v}}{k m_{K}+\frac{K_{L\{R\}}}{v}} * z_{K}\right)$

$882 V^{B_{L\{R\}}^{I}}=\min \left(\frac{\operatorname{vmax}_{G} * \frac{G_{L\{R\}}}{v}}{k m_{G}+\frac{G_{L\{R\}}}{v}} * z_{G}, \frac{\operatorname{vmax}_{I} * \frac{I_{L\{R\}}}{v}}{k m_{I}+\frac{I_{L\{R\}}}{v}} * z_{I}\right)$

Diffusion Rate

$d[\mathrm{~L} / \mathrm{hr}]$

Fluxes:

883 Consumption

$884 \quad V_{B_{L\{R\}}^{K}}^{G}=\frac{V^{B_{L\{R\}}^{K}}}{z_{G}}$

$885 \quad V_{B_{L\{R\}}^{I}}^{G}=\frac{V^{B_{L\{R\}}^{I}}}{z_{G}}$ 
$886 \quad V_{B_{L\{R\}}^{K}}^{K}=\frac{V^{B_{L\{R\}}^{K}}}{z_{K}}$
$887 \quad V_{B_{L\{R\}}^{I}}^{I}=\frac{V^{B_{L\{R\}}^{I}}}{Z_{I}}$

888 Leakage

$889 \quad V_{B_{L\{R\}}^{I}}^{K}=V^{B_{L\{R\}}^{I} * y_{K}}$

$890 \quad V_{B_{L\{R\}}^{K}}^{I}=V^{B_{L\{R\}}^{K}} * y_{I}$

891 Diffusion

$892 \quad V_{D}^{G}=d *\left(\frac{G_{L}}{v}-\frac{G_{R}}{v}\right)$

$893 \quad V_{D}^{K}=d *\left(\frac{K_{L}}{v}-\frac{K_{R}}{v}\right)$

$894 \quad V_{D}^{I}=d *\left(\frac{I_{L}}{v}-\frac{I_{R}}{v}\right)$

895 Dynamics:

$896 \frac{d G_{L\{R\}}}{d t}=-V_{B_{L\{R\}}^{K}}^{G} * B_{L\{R\}}^{K}-V_{B_{L\{R\}}^{I}}^{G} * B_{L\{R\}}^{I}-\{+\} V_{D}^{G}$

$897 \frac{d K_{L\{R\}}}{d t}=-V_{B_{L\{R\}}^{K}}^{K} * B_{L\{R\}}^{K}+V_{B_{L\{R\}}^{I}}^{K} * B_{L\{R\}}^{I}-\{+\} V_{D}^{K}$

$898 \quad \frac{d I_{L\{R\}}}{d t}=-V_{B_{L\{R\}}^{I}}^{I} * B_{L\{R\}}^{I}+V_{B_{L\{R\}}^{K}}^{I} * B_{L\{R\}}^{K}-\{+\} V_{D}^{I}$

$899 \quad \frac{d B_{L\{R\}}^{K}}{d t}=V^{B_{L\{R\}}^{K}} * B_{L\{R\}}^{K}$

$900 \quad \frac{d B_{L\{R\}}^{I}}{d t}=V^{B_{L\{R\}}^{I}} * B_{L\{R\}}^{I}$ 


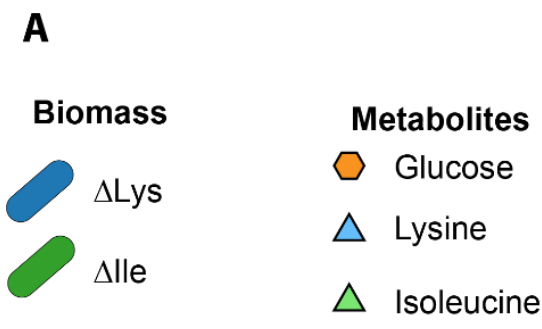

B

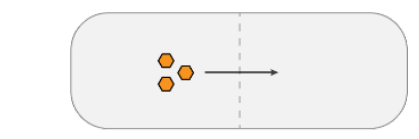

\section{C}
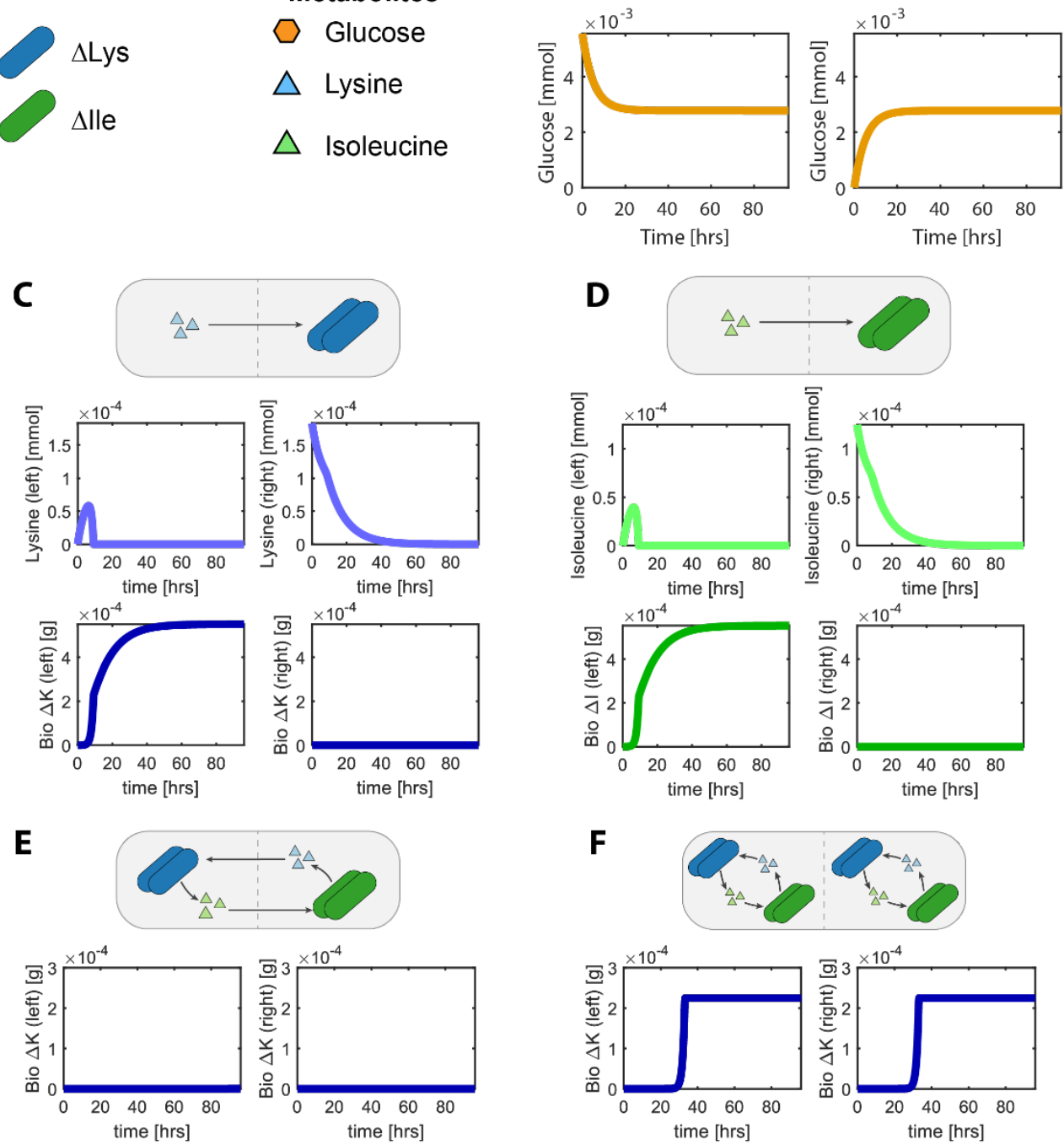

$\mathbf{F}$
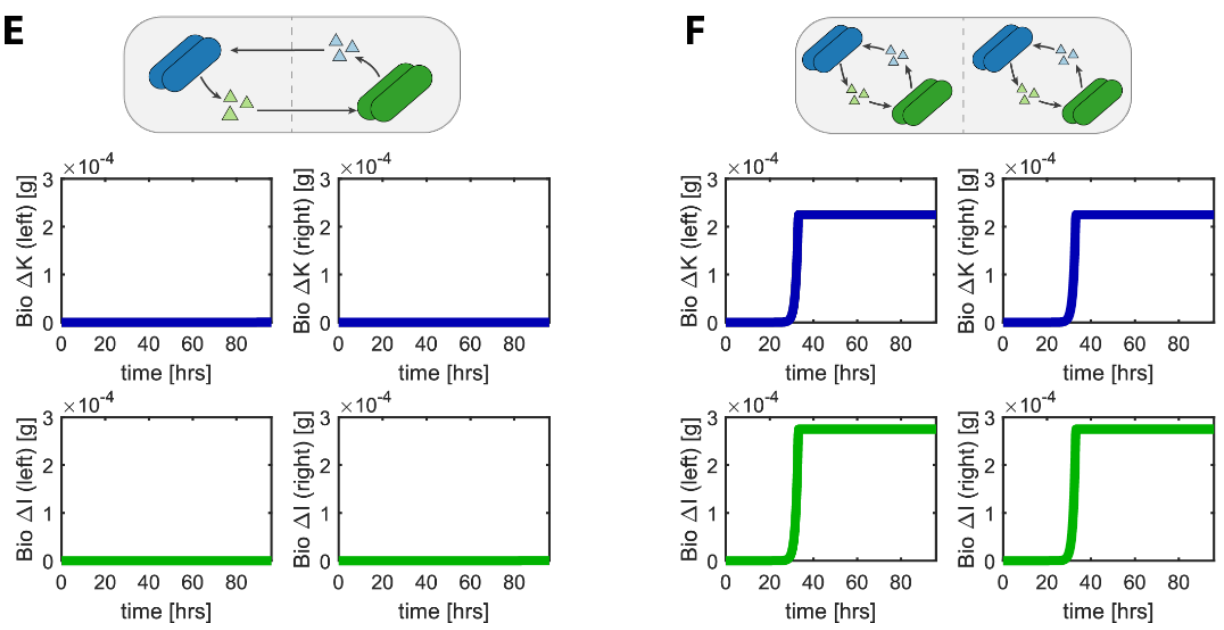

\section{Supplemental Figure S6.1: Simulation of $E$. coli amino acid auxotroph experiments}

903 Different experiments can be simulated by changing the initial conditions and parameters of the model. (A) The legend describes the variables in the dynamic model. These variables appear in the schematic above each experiment, describing the layout of the experiment performed. (B) Chemical diffusion: glucose is added to one side of the device, and diffuses across the membrane until the concentration is equal on both sides. (C\&D) Amino acid diffusion: Auxotrophic E. coli strains are placed on one side of the membrane with their auxotrophy amino acid on the opposite side (C - Lysine, D - Isoleucine). Growth is observed as the amino acid diffuses across the membrane and is taken up by E. coli. (E, F) Auxotroph co-culture: Auxotrophic E. coli strains are placed on the opposite (E) or same (F) sides of the device with no initial amino acid. Growth is observed to be much faster in the same well case (F) than the opposite well case (E). 
961

962

\section{Supplemental Equations S3: Parameters and Initial Conditions}

Literature derived parameters:

Kinetics

These parameters were estimated from the literature as in Harcombe et al. cell reports 2014 (11); the original source referenced in this paper was Gosset, microbial cell factories 2005 (62).

$$
\begin{array}{ll}
> & \operatorname{vmax}_{G}=10[(\mathrm{mmol} \mathrm{G}) /(\mathrm{hr} *(\text { grams } E . \text { coli }))] \\
> & \operatorname{vmax}_{K}=10[(\mathrm{mmol} \mathrm{K}) /(\mathrm{hr} *(\text { grams } E . \text { coli }))] \\
> & \operatorname{vmax}_{I}=10[(\mathrm{mmol} \mathrm{I}) /(\mathrm{hr} *(\text { grams } E . \text { coli }))] \\
> & k m_{G}=0.01[(\mathrm{mmol} \mathrm{G}) / \mathrm{Liter}] \\
> & k m_{K}=0.01[(\mathrm{mmol} \mathrm{K}) / \mathrm{Liter}] \\
> & k m_{I}=0.01[(\mathrm{mmol} \mathrm{I}) / \mathrm{Liter}]
\end{array}
$$

Biomass Stoichiometry

Yeild of E. coli on glucose: 0.5 [grams E. coli / gram glucose] (from BioNumbers 105318, Shiloach et al. Biotechnol Adv. 2005)(63)

Molar mas of glucose: 180.156e-3 [grams glucose / mmol glucose]

$$
>z_{G}=0.0901 \text { [grams E. coli / mmol glucose] }
$$

Yeild of E. coli on amino acids (from Mee et al. PNAS. 2014 supplementary dataset S01) (44):

$1.1 \mathrm{e} 8$ [\# K / cell E. coli]

7.5e 7 [\# I / cell E. coli]

Avogadro's number:

$6.022 \mathrm{e} 20$ [\# / mmol]

Gram / cell E. coli (from cell biology by the numbers "how big is an E. coli cell and what is its mass" cell mass varies with growth rate and the number chosen is in the middle of the E. coli growth rate range)

(64):

500e-15 [gram E. coli / cell E. coli]

$>z_{K}=2.7373[\mathrm{~g}$ E. coli $/ \mathrm{mmol} \mathrm{K}]$

$>Z_{I}=4.0148[\mathrm{~g}$ E. coli $/ \mathrm{mmol} \mathrm{I}]$

Initial Conditions:

Glucose

Stock solution

20 [g glucose] / 100 [mL stock]

Concentration

$2[\mathrm{~mL}$ stock] / $100[\mathrm{~mL}$ medium]

Molecular weight

$180.156 \mathrm{e}-3$ [g/mmol]

\section{Volume}

250e-3 [mL medium]

$$
>G=5.55 * 10^{-3}[\mathrm{mmol}](\text { glucose })
$$

Lysine

Yeild of E. coli on amino acids (from Mee et al. PNAS. 2014 supplementary dataset S01) (44):

$1.1 \mathrm{e} 8[$ [\# K cell E. coli]

Targeted cell count of $10^{9}$

Avogadro's number:

$6.022 \mathrm{e} 20$ [\# / mmol]

$$
>K=1.83 * 10^{-4}[\mathrm{mmol}](\text { Lysine })
$$


965 Yeild of E. coli on amino acids (from Mee et al. PNAS. 2014 supplementary dataset S01) (44):

$9667.5 \mathrm{e} 7$ [\# I / cell E. coli]

967 Targeted cell count of $10^{9}$

968 Avogadro's number:

$9696.022 \mathrm{e} 20[\# / \mathrm{mmol}]$

$970>I=1.25 * 10^{-4}[\mathrm{mmol}]$ (Isoleucine)

971

972 Biomass $\Delta$ Lysine and $\Delta$ Isoleucine

973 Average final OD from positive controls: 0.635 [OD]

974 Theoretical yield from glucose stoichiometry: $5.00 * 10^{-4}$ [g E. coli]

975 Average initial OD from negative controls: 0.151 [OD]

976 Average blank OD from negative controls: 0.139 [OD]

977 Initial biomass:

Stock culture OD600: 0.1

Blank media OD600: 0.04

Inoculation dilution: $1 / 100$

Rough calibration of $E$. coli OD to cells $/ \mathrm{mL}: 8^{*} 10^{8}$ cell $/ \mathrm{mL} / 1$ OD 600

Gram / cell E. coli (from cell biology by the numbers "how big is an E. coli cell and what is its mass" cell mass varies with growth rate and the number chosen is in the middle of the $E$. coli growth rate range): 500e-15 [gram E. coli / cell E. coli]

$$
\frac{5.001 * 10^{-4}[\mathrm{~g} \mathrm{E.coli}]}{0.6349-0.1389[O D]} *(0.1511-0.1389[O D])
$$

$$
>B^{K}=6 * 10^{-8}[\mathrm{~g}](\text { Biomass of } E \text {. coli } \Delta \text { Lysine })
$$

Biomass $\Delta$ Isoleucine

Same inoculation amount as $\Delta$ Lysine

$$
>B^{I}=6 * 10^{-8}[\mathrm{~g}] \text { (Biomass of } E \text {. coli } \Delta \text { Isoleucine) }
$$

Experimentally derived parameters:

Volume

$v=250 * 10^{-6}[\mathrm{~L}]$ 
1000 Supplemental Equations S4: Statistic used for approximate Bayesian computation

1001

1002

$S_{K}=\log _{10}\left(\frac{\left(B_{L, P C}^{I}(t=48)+B_{L, P C}^{K}(t=48)+B_{R, P C}^{I}(t=48)+B_{R, P C}^{K}(t=48)\right) / 2}{B_{L, E x p}^{K}(t=48)}\right)$

1003

$S_{I}=\log _{10}\left(\frac{\left(B_{L, P C}^{I}(t=48)+B_{L, P C}^{K}(t=48)+B_{R, P C}^{I}(t=48)+B_{R, P C}^{K}(t=48)\right) / 2}{B_{R, E x p}^{I}(t=48)}\right)$

1004

1005 Statistic used for equal leakage:

$1006 S_{K, I}=\frac{S_{K}+S_{I}}{2}$ 

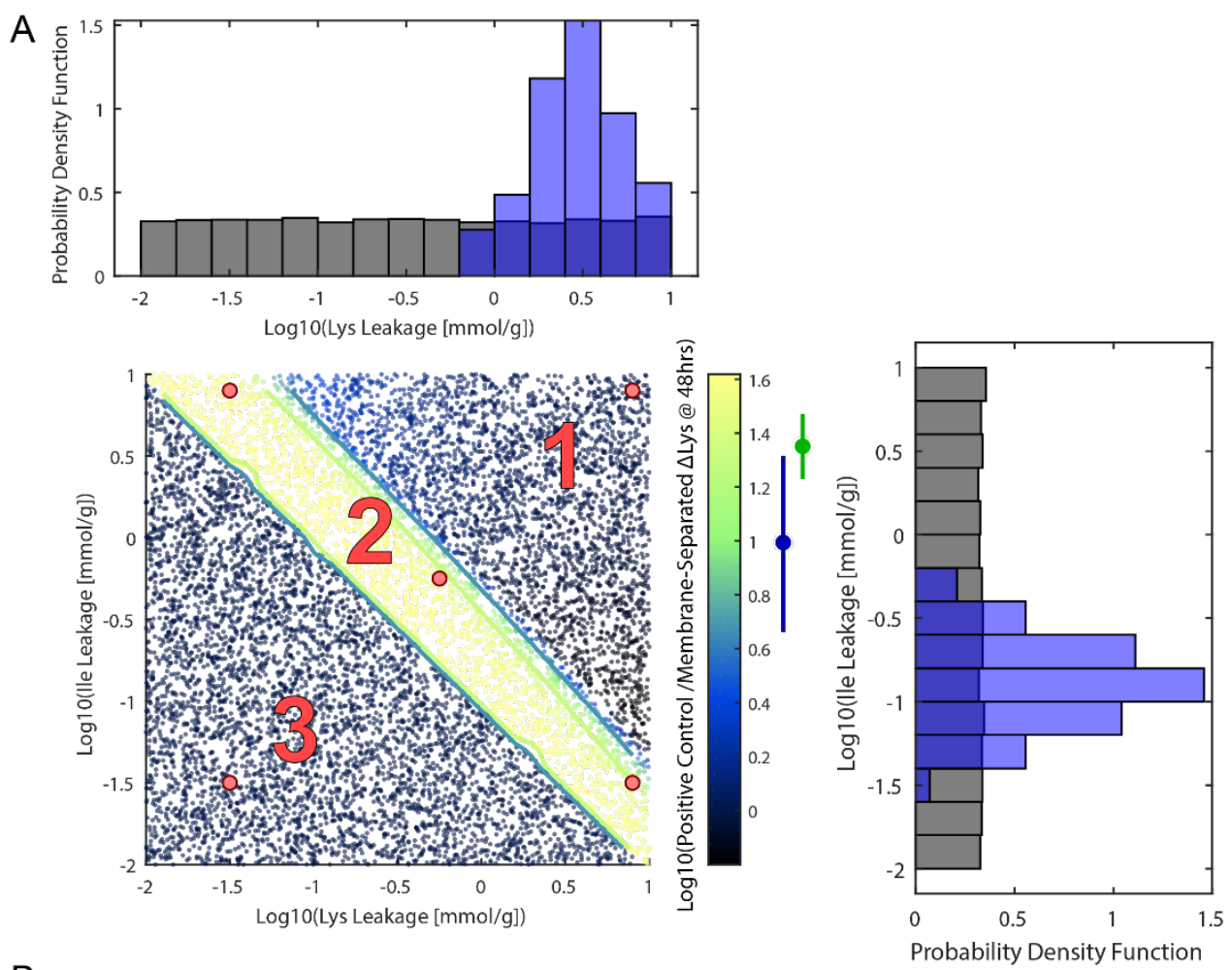

B
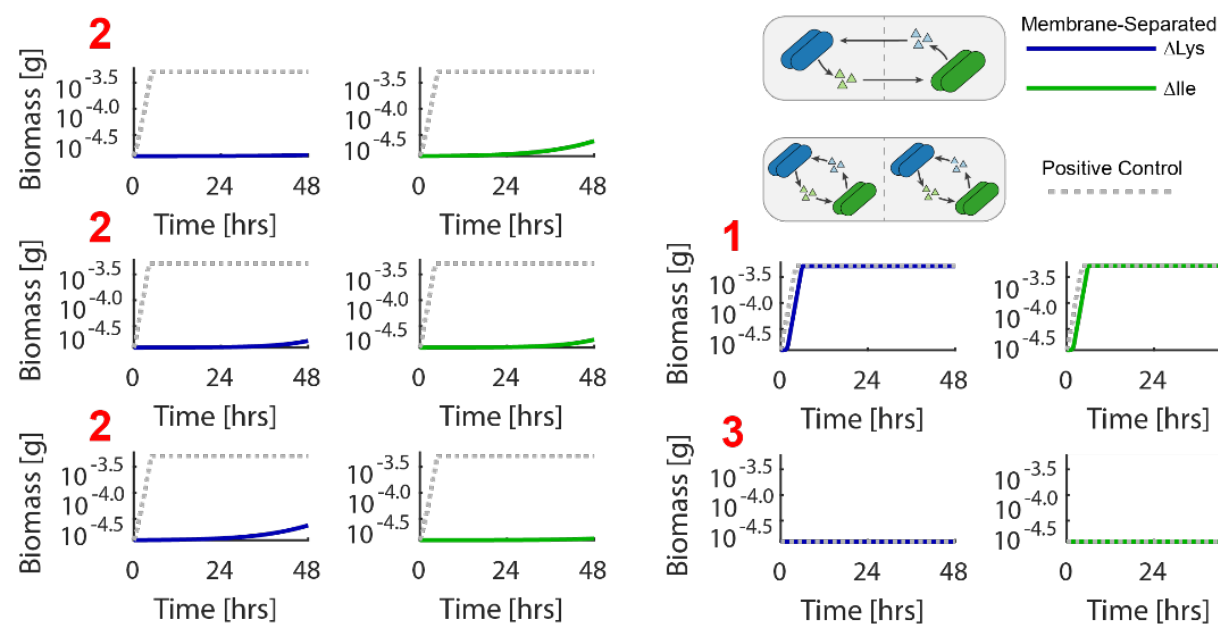

\section{Supplemental Figure S7.1: Sampling of unequal leakage parameters}

1010 (A) Sample space of $\Delta$ Lys and $\Delta$ Ile leakage. The statistic used for approximate Bayesian computation,

$1011 \log$ base 10 of the ratio between positive control growth and membrane-separated growth at 48 hours, is 1012 shown by the color bar. Here the ratio for $\Delta$ Lys is shown. The $\Delta$ Ile ratio plot looks similar with $\mathrm{x}$ and $\mathrm{y}$ 1013 axis transposed. The experimentally mean observed $\Delta$ Lys ratio for pore sizes of $0.03,0.1$, and $0.2 \mu \mathrm{m}$ was 10140.993 (blue dot next to color bar) and the $\Delta$ Ile ratio was 1.37 (green dot next to color bar). Thresholds 1015 equal to twice the experimental statistic standard deviation ( 0.314 for $\Delta$ Lys: blue line next to color bar, 1016 and 0.105 for $\Delta$ Ile: green line) were used to approximate the posterior distribution of the leakages 1017 (histograms, black - prior distribution, blue - posterior distribution). (B) Example growth curves for 1018 different regions of the parameter space. 

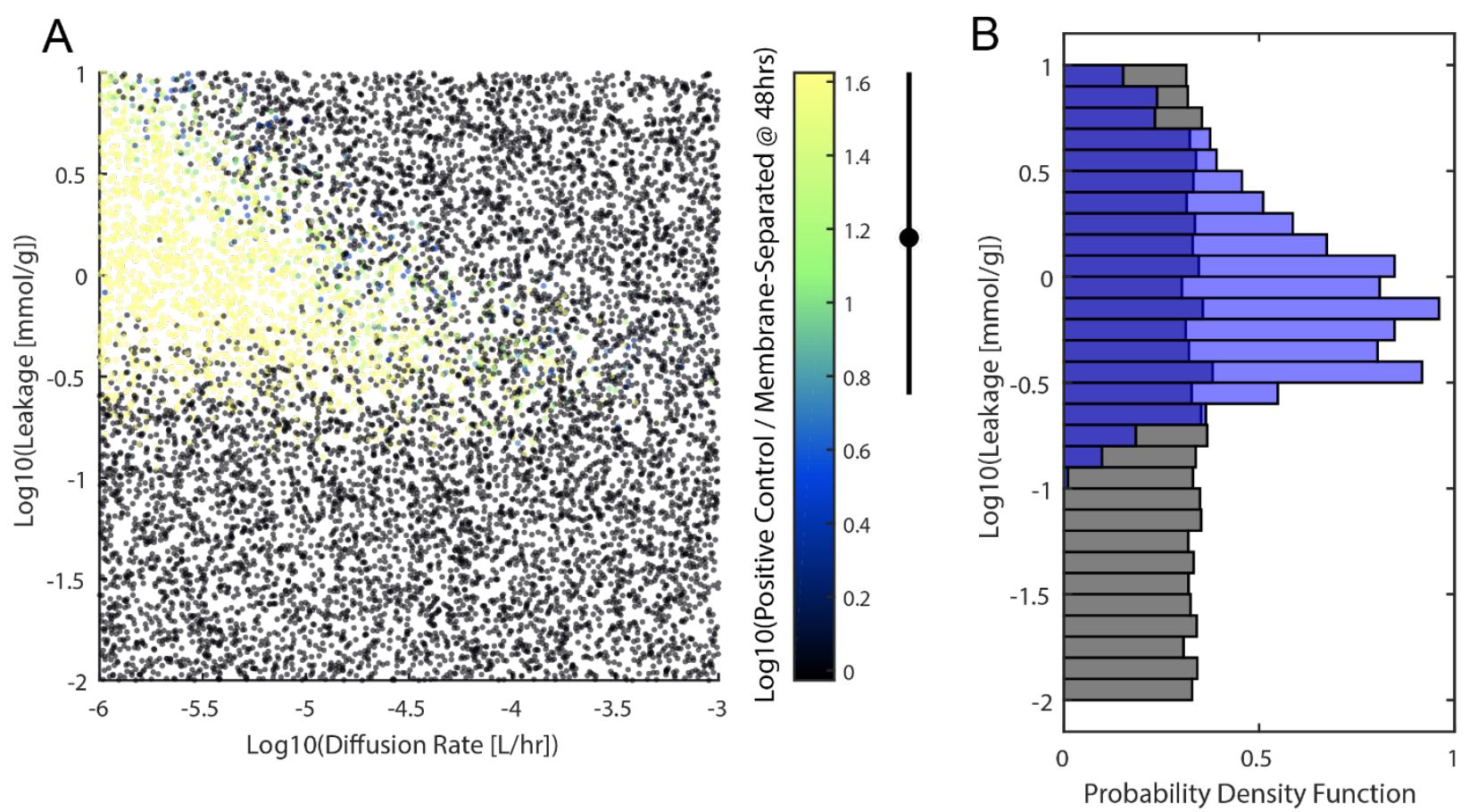

1021

Supplemental Figure S7.2: Sampling of diffusion and leakage parameters with noise

1023 All previously fixed parameters (excluding volume) were randomly sampled from a log uniform

1024

1025 distribution that varied by 1 order of magnitude around the originally fixed literature estimated value. (A)

1026

1027 Sampled space of diffusion and leakage parameters. (B) Applying an approximate Bayesian computation approach the posterior distribution of the leakage parameter was determined (black - prior distribution, blue - posterior distribution). 

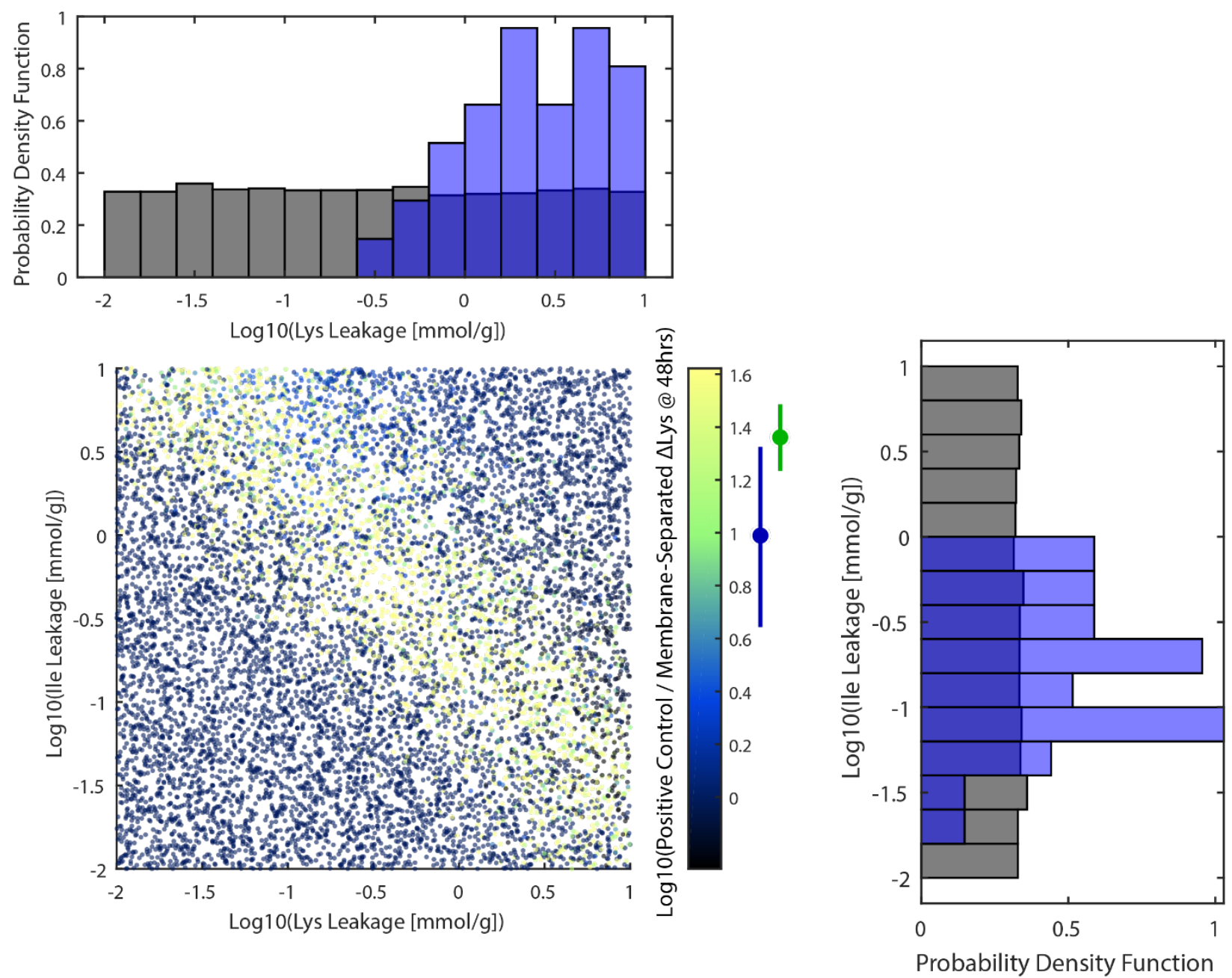

\section{Supplemental Figure S7.3: Sampling of unequal leakage parameters with noise}

1030 All previously fixed parameters (excluding volume) were randomly sampled from a log uniform distribution that varied by 1 order of magnitude around the originally fixed literature estimated value. The sampled space of unequal leakage parameters is shown. Applying an approximate Bayesian computation approach the posterior distribution of the leakage parameters were determined (black - prior distribution,

1034 blue - posterior distribution). 